
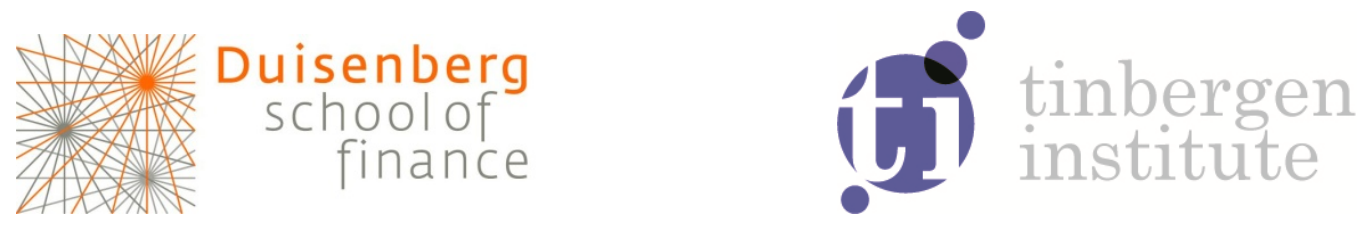

Duisenberg school of finance - Tinbergen Institute Discussion Paper

TI 12-053/2/DSF 34

\title{
Long-Term versus Short-Term Contingencies in Asset Allocation
}

Mahmoud Botshekan'

André Lucas ${ }^{1,2,3}$

1 Faculty of Economics and Business Administration, VU University Amsterdam;

2 Tinbergen Institute;

3 Duisenberg school of finance. 
Tinbergen Institute is the graduate school and research institute in economics of Erasmus University Rotterdam, the University of Amsterdam and VU University Amsterdam.

More TI discussion papers can be downloaded at http://www.tinbergen.nl

Tinbergen Institute has two locations:

Tinbergen Institute Amsterdam

Gustav Mahlerplein 117

1082 MS Amsterdam

The Netherlands

Tel.: +31(0)205251600

Tinbergen Institute Rotterdam

Burg. Oudlaan 50

3062 PA Rotterdam

The Netherlands

Tel.: +31(0)10 4088900

Fax: +31(0)104089031

Duisenberg school of finance is a collaboration of the Dutch financial sector and universities, with the ambition to support innovative research and offer top quality academic education in core areas of finance.

DSF research papers can be downloaded at: http://www.dsf.nl/

Duisenberg school of finance

Gustav Mahlerplein 117

1082 MS Amsterdam

The Netherlands

Tel.: +31(0)20 5258579 


\title{
Long-Term versus Short-Term Contingencies in Asset Allocation*
}

\author{
Mahmoud Botshekan ${ }^{(a)}$, Andre Lucas ${ }^{(a, b)}$ \\ (a) Department of Finance, VU University Amsterdam \\ (b) Duisenberg School of Finance and Tinbergen Institute
}

May 14, 2012

\begin{abstract}
We determine the importance of long-term and short-term components of state variables for asset allocation decisions. The long-term and short-term decompositions are performed using a variety of filtering techniques. We allow for a flexible semiparametric form of the dependence of asset allocation decisions on state variable components. To account for short-sale restrictions, we extend the regular GMM moment conditions with the appropriate Lagrange-Kuhn-Tucker multipliers. Empirically, we find that investors can benefit from reacting differently to short-term versus long-term dynamics of state variables. The induced allocation decisions are implemented in an investment backtest. We find significant improvements in terms of out-of-sample Sharpe ratios and expected utilities for state variables such as the dividend yield and stock market trend.
\end{abstract}

Keywords: Portfolio choice, long and short-term asset allocation, trend-cycle decomposition, GMM under short-sale constraints.

JEL codes: G11.

${ }^{*}$ We would like to thank seminar participants at Duisenberg School of Finance for helpful comments. Andre Lucas thanks the Dutch Science Foundation (NWO) and Mahmoud Botshekan thanks Iran Ministry of Science, Research and Technology for financial support. Corresponding author: Andre Lucas, VU University Amsterdam, FEWEB/FIN, de Boelelaan 1105, 1081 HV Amsterdam, Netherlands, phone: +31 20598 6039, fax: +31 20 598 6020, email: a.lucas@vu.nl. 


\section{Introduction}

By and large, there appears to be consensus in the finance literature that returns are to some extent predictable. ${ }^{1}$ Some of this predictability primarily relates to the cross-section and may be attributed to omitted risk factors such as liquidity in an econometric asset pricing model. The other form of predictability which is our main concern in the current paper relates to the time-series dimension and can be attributed to changing investment opportunity sets, for example due to changing expected returns and/or (co)-variances.

It is already well-known since Merton (1971) that changing investment opportunity sets induce changes in optimal asset allocation decisions, both in a myopic and a dynamic context. In particular, if the changes in the investment opportunity sets can be captured by a (small) set of state variables, the optimal asset allocations also become functions of these state variables. One of the key questions is which state variables provide the most useful information to investors to make their asset allocation decisions.

In this paper, we investigate whether investors can benefit from reacting differently to shortterm versus long-term information in commonly used state variables. Typical examples of state variables used in the literature include dividend yields, default spreads, term spreads, lagged returns, and short rates. Our hypothesis is that, for investors seeking to capture return predictability in their investment decisions, the different short-term versus long-term variation in state variables should not be irrelevant. For example, depending on the investor's horizon, he might react differently to a rise in dividend yields if he knows this rise is due to short-term versus a long-term shift. To investigate how a different reaction to short-term and long-term dynamics in state variables affects portfolio choice, we use state variable decompositions in our asset allocation set-up. Our approach combines two lines of literature: (i) the semiparametric approach of Brandt (1999) and Ait-Sahalia and Brandt (2001) to determine the relative importance of different state variables for asset allocation decisions, and (ii) filtering techniques from the macroeconomic and econometric literature to decompose time-series into their long-term and short-term components. The multivariate approach of the Ait-Sahalia and Brandt (2001) builds on a standard first order condition for a typical investor, where the dependence of the asset allocation on the current state variables is estimated semi-parametrically. The semiparametric nature of the specification allows for much flexibility, but also enables us to use the first order conditions of the asset allocation problem inside a GMM context to determine the

\footnotetext{
${ }^{1}$ See for example Cochrane (1999), Campbell and Viceira (1999), Lettau and Ludvigson (2005) Ang and Bekaert (2007), Cochrane (2008) and Van Binsbergen and Koijen (2010). .
} 
importance of specific state variables.

The novelty in our approach lies in decomposing commonly used state variables from the literature into their long-term and short-term components, rather than in proposing new state variables per se. The long-term and short-term components are used as (new) state variables in the original asset allocation problem. Decomposing time-series into long- and short-term components has a long history in time-series analysis, particularly in macroeconomics. A wide variety of techniques have been proposed in the literature, including the familiar filter of Hodrick and Prescott (1997), newer bandpass filters like Christiano and Fitzgerald (2003), and approaches based on unobserved components models like Harvey (1990) and Harvey and Jaeger (1993). Each of these methods has its own advantages and drawbacks. In our current paper, we focus on the more recent filtering techniques of Christiano and Fitzgerald (2003). The main advantage for our current purposes is that this filter allows us to be explicit on what we label as the short-term or cyclical component of a state variable. In particular, the notion of short-term may have a different connotation for different investors and, as such, may depend on the investment horizon of the decision maker. The Christiano-Fitzgerald filter allows us to investigate the sensitivity of our results to the definition of the short-term component.

As a side contribution of the current paper, we extend the approach of Ait-Sahalia and Brandt (2001) to account for short-sale constraints. The inclusion of short-sale constraints is important to prevent unrealistic asset allocations and leverage. However, short-sale restrictions transform the standard asset allocation problem into a constrained problem. As a result, the first order conditions include the appropriate Lagrange-Kuhn-Tucker multipliers. If these multipliers are omitted, the standard first order conditions are incorrect. As the first order conditions are the prime ingredient for the moment conditions in the GMM estimation stage, omission of the multipliers may lead to a biased inference on the relative importance of longterm versus short-term components for asset allocation. We explain how the multipliers can easily be included in the analysis to avoid this problem.

We apply our approach to a portfolio choice problem involving three assets: stocks, bonds, and a riskfree asset. We use US data for the period April 1953 to June 2011. We find that the short-term components of state variables like the dividend yield, stock market trend and short rate receive a relatively larger weight in the asset allocation decisions than their long-term counterparts. The result is robust to definitions of the short-term from 6 up to 24 months. We can often reject the hypothesis that the investor should react to the aggregate effect of the state variable rather than to its long-term and short-term components separately.

To check the economic significance of our results, we implement the induced investment 
strategies in a backtesting framework, both in-sample and out-of-sample. For the in-sample exercise, we take our estimates for the asset allocation decisions as functions of the longterm and short-term components of each state variable and check how the resulting strategies compare to similar strategies that consider the non-decomposed state variable only. We find substantial improvements in terms of both Sharpe ratios and certainty equivalents for state variables such as the dividend yield and stock market trend. The improvements remain robust in the out-of-sample test.

We subject our benchmark results to a range of robustness checks. In particular, we consider the sensitivity of the results to the decomposition technique used by considering the HodrickPrescott filter as an alternative. The results are very similar to the results obtained with the Christiano-Fitzgerald filter. We also check the sensitivity of the results to the investor's risk aversion parameter. We find that different reaction to short-term versus long-term information persists. We also study the effect of applying the conditional investment strategies using a 3month investment horizon. This is particularly important in our context because the investor's reaction to long-term versus short-term components could depend on the investment horizon. As expected, we find that the long-term component of state variables takes a more important role in the asset allocation decision of longer-term investors.

The remainder of this paper is organized as follows. In Section 2, we introduce the methodology for estimating the optimal portfolio weights under short-sale constraints and explain the filtering techniques used for the decomposition of state variables into their short-term and long-term components. Section 3 describes the data and the decomposition results for the state variables. In Section 4, we provide the empirical estimation results for the relative importance of short-term versus long-term components in asset allocation decisions. We also test the effectiveness of induced investment strategies in a backtesting framework. In Section 5, we provide empirical results for a number of robustness checks. Section 6 concludes. A number of more technical computational issues as well as additional results are gathered in the Appendix.

\section{Methodology}

In this section we first recapitulate the semiparametric approach of Ait-Sahalia and Brandt (2001) for optimal asset allocation decisions. Next, we modify the original moment conditions of Ait-Sahalia and Brandt (2001) by appropriately accounting for the Kuhn-Tucker multipliers that enter the moment conditions of the asset allocation problem under short-sale constraints. The last part of this section explains the different decomposition methods used to disentangle 
our state variables into their short-term and long-term components.

\subsection{Optimal portfolio weights}

We consider the pure asset allocation problem and abstract from intermediate consumption. Our single-period investor is endowed with a power utility function $U\left(W_{t+1}\right)=(1-\gamma)^{-1} W_{t+1}^{1-\gamma}$, where $W_{t+1}$ is time $t+1$ wealth, and $\gamma$ denotes the risk aversion parameter. The investor picks an asset allocation $x_{t} \in \mathbb{R}^{1 \times m}$ by maximizing expected utility,

$$
\max _{x_{t}} \mathrm{E}\left[U\left(W_{t}\left(R_{f}+x_{t} \widetilde{R}_{t+1}\right)\right) \mid Z_{t}\right]
$$

where $R_{f}$ denotes the riskfree rate, $Z_{t} \in \mathbb{R}^{k \times 1}$ denotes a vector of state variables, $\widetilde{R}_{t+1} \in \mathbb{R}^{m \times 1}$ denotes the vector of risky asset returns. For simplicity, we assume for the moment that there is only one risky asset $(m=1)$ such that $x_{t}$ and $\widetilde{R}_{t+1}$ are scalars. This assumption is relaxed later on.

The asset allocation $x_{t}$ solves the standard first order condition

$$
\mathrm{E}\left[m_{t+1} \mid Z_{t}\right]=0
$$

where

$$
m_{t+1}=U^{\prime}\left(W_{t}\left(R_{f}+x_{t} \widetilde{R}_{t+1}\right)\right) \cdot W_{t} \cdot \widetilde{R}_{t+1}
$$

This implies that $x_{t}$ is a function of the state variables $Z_{t}$. The precise functional form of the relationship between $Z_{t}$ and $x_{t}$ is governed by the shape of the utility function and the distribution of the risky returns conditional on $Z_{t}$. Rather than to assume a particular choice for the latter, Brandt (1999) and Ait-Sahalia and Brandt (2001) propose to estimate $x_{t}=x\left(Z_{t}\right)$ semi-parametrically. This avoids many potential biases due to mis-specification of an assumed functional relationship between $Z_{t}$ and $x_{t}$. A fully nonparametric approach to estimating the relation between $Z_{t}$ and $x_{t}$, however, is generally cumbersome, as it is highly data intensive due to the 'curse of dimensionality' and may produce instable results for the asset allocation decisions in general; see also the discussion in Ait-Sahalia and Brandt (2001). A solution to these drawbacks is to use a semiparametric approach, where $x_{t}$ depends on a linear combination of the elements of $Z_{t}$, say $\beta^{\prime} Z_{t}$ with $\beta \in \mathbb{R}^{k \times 1}$, rather than on $Z_{t}$ itself. The advantage is clear: rather than $x_{t}$ being a function that depends on $k$ coordinates, $x_{t}$ now depends on a univariate coordinate $\beta^{\prime} Z_{t}$ only.

The first step in estimating the functional form of $x\left(\beta^{\prime} Z_{t}\right)$ is to replace the objective function 
(1) and the first order condition (2) by their sample analogues,

$$
\max _{x(z)} \sum_{t=1}^{n} \omega_{t}(z) U\left(W_{t}\left(R_{f}+x(z) \widetilde{R}_{t+1}\right)\right),
$$

and

$$
\sum_{t=1}^{n} \omega_{t}(z) m_{t+1}(z)=0,
$$

where $n$ denotes the number of observations, $m_{t+1}(z)$ is defined in (2) with $\beta^{\prime} Z_{t}$ replaced by $z$, and $\omega_{t}(z)=\omega\left(\beta^{\prime} Z_{t}, z\right)$ denotes a weighting function. The weights $\omega\left(\beta^{\prime} Z_{t}, z\right)$ depend on the distance of $z$ to $\beta^{\prime} Z_{t}$. If $\beta^{\prime} Z_{t}$ is closer to $z$, then this observation contains more information on the optimal asset allocation $x(z)$ and the corresponding weight $\omega_{t}(z)$ is higher. As a result, the observation receives a higher weight in both (1) and (2) and thus has a higher impact on $x(z)$. This is most easily seen if $\beta^{\prime} Z_{t}$ can only take one out of two possible values, say $z_{1}$ and $z_{2}$. In that case, equation (1) yields $x\left(z_{1}\right)$ and $x\left(z_{2}\right)$, where the first solution is only based on those observations for which $\beta^{\prime} Z_{t}=z_{1}$, and the latter on the remaining observations. In our empirical analysis in Section 4, the weights are based on a standard normal kernel. More details are provided in the Appendix.

The result of solving equation (4) or (5) is an asset allocation for every value of $z$. If the weights $\omega_{t}(z)$ and the distribution of the state variables are sufficiently smooth, then $x(z)$ is a smooth function of $z$. Note that this requires the vector $\beta$ of index weights to be known. Of course, $\beta$ needs to be estimated as well. We first note that $\beta$ is not identified given that we estimate $x(z)$ non-parametrically. In particular, the non-parametric approach to estimating $x(z)$ makes any intercept term in $\beta^{\prime} Z_{t}$ as well as the length of $\beta$ unidentified. We therefore assume that $Z_{t}$ does not contain a constant term, and that $\beta$ has unit length, i.e., $\|\beta\|^{2}=\beta^{\prime} \beta=$ 1.

To estimate the parameters $\beta$, we adopt a Generalized Methods of Moments (GMM) approach. Following Ait-Sahalia and Brandt (2001), we use the conditional moment condition (2) to construct the unconditional moment condition

$$
\mathrm{E}\left[m_{t+1}\left(\beta^{\prime} Z_{t}\right) \otimes g\left(Z_{t}\right)\right]=0
$$

where $m_{t+1}\left(\beta^{\prime} Z_{t}\right)$ is defined in (3) and depends on the semiparametric estimate of the asset allocation $x\left(\beta^{\prime} Z_{t}\right), g\left(Z_{t}\right)$ is a known deterministic vector function of $Z_{t}$, and $\otimes$ denotes the Kronecker product. In our empirical work later on, we use $g\left(Z_{t}\right)=\left(1, Z_{t}^{\prime}\right)^{\prime}$. Ait-Sahalia and Brandt (2001) argue that this choice for $g(\cdot)$ is adequate and numerically efficient in the asset allocation context. 
To implement the GMM estimator for $\beta$, we define

$$
\bar{m}=\frac{1}{n} \sum_{t=1}^{n} m_{t+1}\left(\beta^{\prime} Z_{t}\right) \otimes g\left(Z_{t}\right)
$$

and the GMM objective function

$$
\min _{\|\beta\|=1} \bar{m}^{\prime} W \bar{m}
$$

where $W$ is an appropriate positive definite weighting matrix. Following Hansen (1982), the (infeasible) optimal choice of the weighting matrix is given by

$$
W=\mathrm{E}\left[m_{t+1} m_{t+1}^{\prime} \otimes g\left(Z_{t}\right) g\left(Z_{t}\right)^{\prime}\right]^{-1}
$$

We take a feasible multi-step approach to proxy the optimal choice of $W$. We start by setting $W=\mathrm{I}$ and obtain a first-step estimate of $\beta$, say $\hat{\beta}^{(1)}$. We use this estimate to update the weighting matrix to

$$
\hat{W}^{(1)}=\left(\frac{1}{n} \sum_{t=1}^{n} m_{t+1}\left(\left(\hat{\beta}^{(1)}\right)^{\prime} Z_{t}\right) m_{t+1}\left(\left(\hat{\beta}^{(1)}\right)^{\prime} Z_{t}\right)^{\prime} \otimes g\left(Z_{t}\right) g\left(Z_{t}\right)^{\prime}\right)^{-1}
$$

The matrix $\hat{W}^{(1)}$ is then used as the new weighting matrix in (8) to obtain a new estimate $\hat{\beta}^{(2)}$. The process is repeated until convergence. Usually, three or four iterations suffice. Standard errors are computed based on the converged pair $\left(\hat{\beta}^{(i)}, \hat{W}^{(i)}\right)$.

\subsection{Imposing short-sale constraints}

A plain vanilla implementation of the GMM estimator and asset allocation procedure as described in Section 2.1 typically results in optimal allocations with unrealistic short-selling of particular asset classes, thus obstructing a sensible interpretation of our results. To avoid such problems, we impose short sale constraints. Such constraints, however, cannot be merely imposed ex post after solving the unconstrained problem (4) or (5). In particular, moment condition (2) no longer holds under short sale restrictions. For example, if an asset allocation lies on the boundary, (2) is in general strictly negative, as decreasing the asset weight would increase the objective function further. As the moment conditions also directly enter the GMM criterion (7) and (8), not accounting for the short-sale constraints explicitly in the moment conditions can affect our estimates of $\beta$ and therefore our interpretation of the impact of specific state variables.

We therefore proceed by adjusting the moment conditions in (2) and (7) by formulating the appropriate Kuhn-Tucker first order conditions for the constrained optimization problem. 
These adjusted moment conditions are then used in (7) and (8) to estimate $\beta$. For details on the numerical implementation, we refer to the Appendix.

As an illustration, we consider the case with one risky and one riskfree asset. The asset allocation problem now becomes

$$
\begin{array}{cl}
\max _{x(z)} & \mathrm{E}\left[U\left(W_{t}\left(R_{f}+x(z) \widetilde{R}_{t+1}\right)\right) \mid \beta^{\prime} Z_{t}=z\right] \\
\text { s.t. } & 0 \leq x(z) \leq 1
\end{array}
$$

The corresponding Lagrangian is given by

$$
\max _{0 \leq x(z) \leq 1} \mathrm{E}\left[U\left(W_{t}\left(R_{f}+x(z) \widetilde{R}_{t+1}\right)\right) \mid \beta^{\prime} Z_{t}=z\right]+\lambda_{1}(z) \cdot x(z)+\lambda_{2}(z) \cdot(1-x(z)),
$$

where $\lambda_{1}(z)$ and $\lambda_{2}(z)$ are the Lagrange multipliers. If we obtain an internal solution, $\lambda_{1}(z)=$ $\lambda_{2}(z)=0$ and we recover the original moment condition from Section 2.1. If, however, the constrained optimum lies at the boundary $x(z)=0$, then the Kuhn-Tucker conditions for a constrained optimum yield $\lambda_{2}(z)=0$ and $\lambda_{1}(z)>0$. Similarly if the optimum lies at the other boundary $x(z)=1$, we obtain $\lambda_{1}(z)=0$ and $\lambda_{2}(z)>0$. Omitting the Lagrange multipliers from the Kuhn-Tucker first order conditions therefore gives the wrong moment conditions to identify $\beta$. The solution is obvious as well. Rather than only computing $x(z)$ from (10), we compute both $x(z)$ and all the relevant Lagrange multipliers $\lambda_{i}(z)$ for $i=0, \ldots, m$ if we have $m$ risky assets and one riskfree asset. The Lagrange multipliers are used to construct the adjusted $\bar{m}$ for $(7)$ as

$$
\bar{m}=\frac{1}{n} \sum_{t=1}^{n} \tilde{m}_{t+1}\left(\beta^{\prime} Z_{t}\right) \otimes g\left(Z_{t}\right)
$$

with

$$
\tilde{m}_{t+1}\left(\beta^{\prime} Z_{t}\right)=U^{\prime}\left(W_{t}\left(R_{f}+x\left(\beta^{\prime} Z_{t}\right) \widetilde{R}_{t+1}\right)\right) \cdot W_{t} \cdot \widetilde{R}_{t+1}+\lambda\left(\beta^{\prime} Z_{t}\right)-\lambda_{0}\left(\beta^{\prime} Z_{t}\right) \iota,
$$

where $\lambda(z)=\left(\lambda_{1}(z), \ldots, \lambda_{m}(z)\right)^{\prime}$ with $\lambda_{i}(z) \in \mathbb{R}^{+} \cup\{0\}$ for $i=0, \ldots, m$, and where $\iota$ or is an $m \times 1$ vector of ones.

\subsection{Decomposing state variables into short and long components}

The main contribution of our paper lies in applying the methodology described in Section 2.1 and 2.2 to a vector $Z_{t}$ of short-term and long-term components of familiar state variables like the dividend yields, term spread, etc. In this way, we can test whether investors can benefit from reacting differently to long-term versus short-term developments in these state variables. 
Decomposing time-series into a trend and a cyclical component has a long history, particularly in macroeconomics. Familiar and well-used techniques are the (nonparametric) HodrickPrescott filter or the more recent Christiano-Fitzgerald filter. Alternative approaches are parametric in nature such as decompositions based on unobserved components time-series models, see Harvey (1990). The benchmark for our analysis is the Christiano-Fitzgerald filter, see Christiano and Fitzgerald (2003) and Baxter and King (1999). We briefly explain each of our filters in the sections below.

\subsubsection{Christiano-Fitzgerald filter}

The Christiano-Fitzgerald filter (CF filter hereafter) is a band pass filter that formulates the trend-cycle decomposition in the frequency domain. In this approach, for each observation, the trend and cyclical components are essentially a weighted moving average of all observations. Christiano and Fitzgerald (2003) and Baxter and King (1999) propose an approximation for the ideal infinite band pass filter for finite samples.

The filter can be calculated as follows. Suppose we want to isolate the cyclical component of $y_{t}=\tau_{t}+c_{t}$, where $\tau_{t}$ and $c_{t}$ denote the trend and cycle, respectively, and the cycle length is between $p_{l}$ and $p_{u}$ months, where $2 \leq p_{l}<p_{u}<\infty$. The CF filter approximation $\hat{c}_{t}$ of cyclical component $c_{t}$ is given by

$$
\hat{c}_{t}=\tilde{B}_{t-1} y_{1}+B_{t-2} y_{2}+\ldots+B_{1} y_{t-1}+B_{0} y_{t}+B_{1} y_{t+1}+\ldots+B_{T-1-t} y_{T-1}+\tilde{B}_{T-t} y_{T}
$$

where $B_{j}=\frac{\sin j b-\sin j a}{\pi j}, j \geq 1, B_{0}=\frac{b-a}{\pi}, a=\frac{2 \pi}{p_{u}}, b=\frac{2 \pi}{p_{l}}$ and $\tilde{B}_{k}=-\frac{1}{2} B_{0}-\sum_{j=1}^{k-1} B_{j}$.

The CF filter approximation of trend component can be written as

$\hat{\tau}_{t}=-\tilde{B}_{t-1} y_{1}-B_{t-2} y_{2}-\ldots-B_{1} y_{t-1}+\left(1-B_{0}\right) y_{t}-B_{1} y_{t+1}-\ldots-B_{T-1-t} y_{T-1}-\tilde{B}_{T-t} y_{T}=y_{t}-\hat{c}_{t}$.

For $p_{l}=2$, The maximum weight for calculating $\hat{\tau}_{t}$ is assigned to $y_{t}$ and is equal to $\left(1-B_{0}\right)$. For other observations, weights have a cyclical pattern with a decreasing amplitude. The weights are symmetric around time $t$. The frequency and amplitude of the cyclical pattern depend on the values of $p_{l}$ and $p_{u}$. The values of $\tilde{B}_{t-1}$ and $\tilde{B}_{T-t}$ are such that the weights for the trend component $\hat{\tau}_{t}$ and the cyclical component $\hat{c}_{t}$ sum to one and zero, respectively. Note that for $p_{l}=2$, we have the simpler form $B_{j}=-\sin (j a) /(\pi j)$, which clearly shows the cyclical nature of the weights with decreasing amplitude for observations on either side of $y_{t}$. 
Obviously, there is no symmetric pattern for $\hat{\tau}_{1}$ and $\hat{\tau}_{T}$ and weights have a one-sided decreasing pattern for the first and last observation. Because the CF filter uses both future and past observations to calculate the trend component $\hat{\tau}_{t}$ for observation $y_{t}$, it works essentially as a smoother rather than a filter. Only for end-of-sample observation, i.e. for computing $\hat{\tau}_{T}$, it behaves as a true filter. Because of this, in our main empirical analysis, we run the filter recursively and compute end-of-sample decomposition for each observation. The drawback of this method is that the CF filter (as well as the HP filter) is optimal for an underlying random walk time series, so much weight is put on the end-of-sample observation. On the other hand, this is a feasible solution to implement the investment strategy in a practical investment context. In the Appendix, we provide additional results using the standard smoother version.

The main advantage of the CF filter is that this filter allows us to be explicit about the definition of the cyclical component (in our context the short-term component of state variables) by using different values for $p_{l}$ and $p_{u}$. We set the minimum value for $p_{l}=2$. In this case, the cyclical component captures variation up to $p_{u}$ months. In our empirical analysis in Section 4 , we use a range of values for $p_{u}$ from 6 to 72 months to test the sensitivity of our results to the definition of the short-term component, as the notion of short-term may have a different connotation for different investors.

\subsubsection{Hodrick-Prescott filter}

The Hodrick-Prescott filter (HP filter hereafter) was originally proposed by Leser (1961) and popularized by Hodrick and Prescott (1997). This filter is widely used in the context of macro data for trend-cycle decompositions. The HP filter decomposes a time series into a trend component and a cyclical component $\left(y_{t}=\tau_{t}+c_{t}\right)$. The trend component is obtained by minimizing the function

$$
\min _{\left\{\tau_{t}\right\}} \sum_{t=1}^{T}\left(y_{t}-\tau_{t}\right)^{2}+\lambda \sum_{t=2}^{T}\left[\left(\tau_{t+1}-\tau_{t}\right)-\left(\tau_{t}-\tau_{t-1}\right)\right]^{2},
$$

where $\lambda \in \mathbb{R}^{+}$is smoothing parameter.

The first part of the optimization function minimizes the sum of squared deviations of the trend component from the original series. The second part minimizes the curvature of the trend series by putting a penalty on second differences of trend component. The solution to the optimization problem therefore requires a tradeoff between smoothness and fit. The tradeoff is governed by the value of $\lambda$. When $\lambda \rightarrow 0$, the trend approximately becomes equal to the original series. When the $\lambda \rightarrow \infty$, the trend becomes linear. 
As Danthine and Girardin (1989) show, the solution of the optimization problem can also be represented by the linear transformation

$$
\hat{\tau}=\left[\mathrm{I}+\lambda K^{\prime} K\right]^{-1} Y
$$

where $\hat{\tau}=\left(\hat{\tau}_{1}, \hat{\tau}_{2} \ldots \hat{\tau}_{T}\right), Y=\left(y_{1}, y_{2}, \ldots y_{T}\right)$, I is a $T \times T$ identity matrix, and $K=\left\{k_{i j}\right\}$ is a $(T-2) \times T$ matrix with elements

$$
k_{i j}= \begin{cases}1 & \text { if } i=j \text { or } i=j+2 \\ -2 & \text { if } i=j+1 \text { is odd } \\ 0 & \text { elsewhere. }\end{cases}
$$

As Equation (17) shows, the trend component $\hat{\tau}_{t}$ for observation $y_{t}$ is computed using the inner product of the $t^{\text {th }}$ row of matrix $\left(\mathrm{I}+\lambda K^{\prime} K\right)^{-1}$ (a vector of weights) and the vector $Y$ (all observations). So similar to the CF filter, the HP filter is also a smoother rather than a filter as it uses both future and past observations to compute the trend component of each observation. Only for the last observation, the filter works as a true filter, see also Razzak (1997). For the results presented in Section 4, we run the filter recursively and compute the end-of-sample decomposition for each observation. In the Appendix, we provide additional results using the standard version of the HP filter.

The main question for applying the HP filter is again which numbers should be used for $\lambda$. Hodrick and Prescott (1997) suggest that for quarterly and monthly data $\lambda=1600$ and $\lambda=$ 14400 are optimal, respectively, but there is no uniform consensus in the empirical literature. In Section 3 and 4, we apply a range of different values for $\lambda$, namely 240, 900, 1600, and 14400, to check the sensitivity of our results to this parameter. The intention is that the decomposition results be comparable with the results obtained using the CF filter.

\section{Data and decomposition results}

\subsection{Data}

We consider three assets: stocks, bonds, and treasury bills. Stock returns are given by the monthly returns on the CRSP value-weighted index for all NYSE, AMEX, and NASDAQ stocks. For bond returns, we use a monthly time-series on 10-Year Treasury Constant Maturity rate from Board of Governors of the Federal Reserve to construct the returns on 10 year government bonds. We construct the return at time $t+1$ by calculating the time $t+1$ present value of 
a 10 year bond issued at part at time $t$ with coupon $r_{t}$ where we discount using rate $r_{t+1}$ announced at $t+1$. The risk free rate is the one-month Treasury bill rate. The returns are sampled at monthly frequency from April 1953 to June 2011. The complete sample consists of 699 observations.

We consider six popular ${ }^{2}$ state variables from the literature: the default spread, the log dividend-to-price ratio of the S\&P index, the term spread, a trend or momentum variable for the S\&P index, the short rate, and the dividend growth rate. The default spread is measured as the yield difference between Moody's Baa- and Aaa-rated corporate bonds. The dividend yield is the sum of dividends paid on the S\&P index over the past 12 months divided by the current level of the index. The term spread is the yield difference between 10-year and 1-year government bonds. The trend variable is the difference between the log of the current S\&P index level and the log of the average index level over the previous 12 months.

The state variables are shown in Figure 1, while Table 1 provides descriptive statistics for the data. Stock and bond returns both appear to be skewed, but to opposite sides. In addition, there is excess kurtosis signalling that returns may be non-normally distributed. Pairwise correlations of the risky assets' returns with the candidate state variables are modest. This is common in this type of analysis: the predictable component of returns is typically small compared to the volatility of the returns.

\subsection{Long-term and short-term components}

Our benchmark decomposition in the analysis below is provided by the Christiano-Fitzgerald filter. A crucial choice in the implementation of this filter is the definition of the cyclical or short-term component. We define the short-term as the period up to $x$ months, where we vary the choice of $x$ from 6 months to 72 months. Clearly, this definition affects the estimated short-term and long-term components, and we investigate the sensitivity of our results to the definition used.

AS a typical illustration, Figures 2 and 3 present the decomposition results for the default spread and the log dividend yield, respectively. The patterns are quite clear. For the shortest definition of the short-term component, $x=6$, the long-term component of each series looks very similar to the original series. The short-term component is close to the innovation in the

\footnotetext{
${ }^{2}$ Similar variables were used in for example Fama and French (1988), Fama and French (1989), Keim and Stambaugh (1986), Kandel and Stambaugh (1996), Barberis (2000), Campbell and Viceira (1999), Brandt (1999), Ait-Sahalia and Brandt (2001), Handa (2006), and Wachter and Warusawitharana (2009).
} 
series since the last month. Moving to longer-term definitions of the short-term component, we see that the long-term component picks up the long-term patterns of the data more, leaving more room for persistent cyclical deviations.

In our robustness analysis in Section 5, we investigate the sensitivity of our results to the filtering techniques used. In particular, we consider the Hodrick-Prescott filter as a possible alternative choice. For this filter, the smoothness of the long-term component is governed by the smoothing parameter $\lambda$. We use four values of $\lambda$, namely $240,900,3600$, and 14400 . The decomposition of the default spread and the log dividend yield are presented in Figure 4.

The patterns obtained for the Christiano-Fitzgerald and the Hodrick-Prescot filter appear quite comparable for given values of the smoothing parameters. We therefore expect the results to be comparable across filters. Rather than the choice of the particular filter, it appears much more important how the smoothing parameter $x$ or $\lambda$ is chosen. The smoothing parameter has a substantial effect on both the long-term and short-term component that are filtered from the data. Rather than fixing the smoothing parameter to one particular value, we consider a range of values and check the sensitivity of our results to the values used.

The decomposition results presented up to now were computed using the entire sample. As both the Christiano-Fitzgerald filter and the Hodrick-Prescot filter are effectively smoothers rather than filters, they use both past and future values of the state variables to compute the decomposition at time $t$. Therefore, they cannot be used in their original form in a real-time asset allocation context. We adopt our decomposition methodology in the following way. For every month in the sample, we run the Christiano-Fitzgerald filter using only the data up to the start of the month. The filter produces our best (filtered) estimate of the long-term and short-term component at the start of the month. These components are stored in the vector $Z_{t}$. The process is repeated for all months from April 1964 till June 2011, each time using data from April 1953 till the start of the current month This gives us a burn-in sample for the filter of 10 years. We call this procedure "recursive filtering" and subsequently use the recursively filtered estimates of long-term and short-term components to estimate the optimal index weights $\beta$ and the performance of the investment strategies.

Figures 5 and 6 present the decomposition results for the default spread and the log dividend yield using the recursive Christiano-Fitzgerald filter. Figure 7 presents similar results for the recursive Hodrick-Prescott filter. It is well-known that the Christiano-Fitzgerald filter (and the Hodrick-Prescott filter as well) are very sensitive to the end-points of the sample. This is due to the non-stationarity of the time series model underlying the filter. Both filters are optimal for an underlying random walk or local trend model, implying that near the end-points much 
weight is put on the most recent observations. We thus expect the results for the recursive filter to different from those for the standard filter. This is clear if we consider the figures that uses a longer definition of the short component, i.e., larger $x$ or smaller $\lambda$. For those filters, the long-term components are less smooth than for the standard filter and have a higher correlation with the original state variables.

The standard deviations of the long-term $\left(\sigma_{L}\right)$ and short-term $\left(\sigma_{S}\right)$ components for the standard and recursive Christiano-Fitzgerald filter are given in Tables 2 and 3, respectively. The tables also contain the angles $\phi=\arctan \left(\sigma_{S} / \sigma_{L}\right)$ such that

$$
Z_{i t}=\cos (\phi) Z_{i t}^{L}+\sin (\phi) Z_{i t}^{S}
$$

where $Z_{i t}^{L}$ is the standardized (by $\sigma_{L}$ ) long-term component of $Z_{i t}$, and $Z_{i t}^{S}$ is the standardized (by $\sigma_{S}$ ) short-term component. The variation $\sigma_{S}$ of the short-term component is generally considerably smaller than that of the long-term component. The only two exceptions are the stock market trend (TR) and the dividend growth (DG). The angles $\phi$ can be used later to test whether the decomposition into a long-term and short-term component is statistically significantly better than using the original non-decomposed state variable.

Before we turn to our GMM estimation results, it is insightful to perform simple preliminary regressions of returns on each of the state variables, as well as on the long-term and short-term components of each state variable. Table 4 presents the results for stock returns. We recognize the familiar low $R^{2}$ values for return regressions. The predictable component of stock returns, if present at all, is typically small in terms of $R^{2}$. Decomposing the state variables into their long-term and short-term components and regressing stock returns on the two components mechanically results in higher $R^{2}$ s. The improvement is, however, sizeable for some filters, particularly for the $\mathrm{CF}(12)$ and $\mathrm{CF}(24)$ filters.

The most interesting feature of the table, however, is the partial $R^{2}$ values. The partial $R^{2}$ measures the contribution of a variable after all other variables have already been included in the model. The partial $R^{2}$ values reveal that for the short rate (SR) and stock market trend (TR), the overall predictability is more attributable to the short-term rather than to the long-term component. The conclusion holds irrespective of the version of the Christiano-Fitzgerald filter used. The short-term component is also dominant for default spread (DS) and log dividend yield (DY), if the $\mathrm{CF}(12)$ or $\mathrm{CF}(24)$ is used. Here we also see the highest improvement of $R^{2}$ values. For the term spread (TS), the contribution of the short-term and long-term component to the overall $R^{2}$ is roughly equal. 
A similar result is presented in Table 5 for bond returns. The overall picture resembles that of Table 4 . The $R^{2}$ s for the non-decomposed state variables are typically low. Decomposing the state variables into their long-term and short-term component improves the $R^{2} \mathrm{~s}$, where the improvements are more visible for the $\mathrm{CF}(6), \mathrm{CF}(12)$, and $\mathrm{CF}(24)$. For these filters, a larger portion of the predictability can be attributed to the short-term component for five out of the six state variables. The exception is the term spread (TS), for which the long-term component has a larger contribution to the predictability for all version of the filter used. The $R^{2} \mathrm{~s}$ for bond returns are also higher than for stock returns. All in all, based on these preliminary regression results we conclude that there is evidence that long-term and short-term dynamics in state variables have a different impact on stock and bond returns. Investors may therefore want to react differently to developments in either component.

\section{Empirical results}

\subsection{Estimation of the component weights $\beta$}

We first turn our attention to the the estimation of $\beta$. Our state-variables are decomposed recursively in a long-term and short-term component as explained in Section 3.2 using the Christiano-Fitzgerald filter. We then consider each state variable separately to assess the relative importance of its components. We assume that the long-term and short-term components are the first and second element of $Z_{t}$, respectively, i.e.,

$$
\left.Z_{t}=\text { (long-term component, short-term component }\right)^{\prime} .
$$

The vector $\beta$ is parameterized as

$$
\beta=(\cos (\phi), \sin (\phi))^{\prime}, \quad 0 \leq \phi \leq \pi
$$

The length of $\beta$ is equal to one by construction, whereas all different combinations of the longterm and short-term components are still possible. To see this, note that values of $\phi \in[\pi, 2 \pi]$ give the same GMM objective function as $\phi-\pi$ due to the non-parametric estimation of the asset weights $x\left(\beta^{\prime} Z_{t}\right)$ in $(7)$.

Before we present the actual results, we highlight some important issues concerning scaling. As seen from the decomposition results in Section 3.2, the long-term components typically have a larger variation than the short-term components. We therefore scale both components by their time-series standard deviation in order for the $\beta$ s to indicate the relative importance 
of the two (scaled) components for decision making. We also demean both components. If $\sigma_{L}$ and $\sigma_{S}$ denote the standard deviations and $\mu_{L}$ and $\mu_{S}$ sample means of the long-term and short-term components, respectively, we change (18) to

$$
Z_{t}=\left(\left(\text { long-term component }-\mu_{L}\right) / \sigma_{L},\left(\text { short-term component }-\mu_{S}\right) / \sigma_{S}\right)^{\prime}
$$

For the special case $\phi=\arctan \left(\sigma_{S} / \sigma_{L}\right), \beta^{\prime} Z_{t}$ is the demeaned sum of the (unscaled) longterm and short-term components. This implies that the non-decomposed state variable can be used for asset allocation rather than its individual components. The setting with the original state variables is thus included as a special case and we can even test statistically whether the decomposition of information into long and short-term components adds to the descriptive ability of the model.

The benchmark results are presented in Table 6. Each column holds the result for a different state variable, while each block holds the results for a different version of the ChristianoFitzgerald filter. The filters differ in their definition of the short-term component. The CF(6) filter for example defines the short-term as being up to 6 months, while the CF(72) filter defines the short-term to last up to 72 months or 6 years.

We start our discussion by looking at the results based on the definition that the shortterm component covers up to 6 months. For $\mathrm{CF}(6)$, the loading of the short-term component $\left(\beta_{2}\right)$ is larger in absolute size than that of the long-term component $\left(\beta_{1}\right)$ for most of the variables, except the default spread and the term spread. The scaled short-term components thus dominate the asset allocation decisions. These results are in line with the preliminary regression results in Tables 4 and 5 where the short-term component appeared to contribute more to the predictability of stocks and bonds returns.

The second thing to notice is that for a number of state variables (DS, DY, DG) the loadings of the long-term and short-term components have opposite signs. For the default spread (DS), this difference in sign is even statistically significant, as the $95 \%$ confidence interval for $\hat{\phi}$ contains neither $\pi$ nor $\pi / 2$. If the two components have opposite signs, this excludes the possibility that the non-decomposed state variable would be optimal for asset allocation. The latter would be a weighted average of the two components with strictly positive weights $\sigma_{L} / \sqrt{\sigma_{L}^{2}+\sigma_{S}^{2}}$ and $\sigma_{S} / \sqrt{\sigma_{L}^{2}+\sigma_{S}^{2}}$. An economic rational for different weights is that investors should react differently to fundamental changes in the economy compared to transitory changes. Long-term fundamental changes directly impact expected returns and therefore optimal asset allocations. Short-term deviations, however, can have less of or even an opposite effect due to an overreaction of markets to short-term information. As a result, such information should be 
exploited differently by a rational investor, depending on his investment horizon.

The robustness of the results with respect to the definition of the short-term component can be inspected by comparing the different blocks in Table 6 . The $\mathrm{CF}(x)$ filter defines the short-term component as the variation attributable to data frequencies up to $x$ months, for $x=6,12,24,36,48,72$. The information from Table 6 is presented graphically in Figure 8 . For each value of $x$, we present the value of the estimated angle $\hat{\phi}$ as a function of $x$, where $\beta_{1}=\cos (\phi)$ and $\beta_{2}=\sin (\phi)$. Each panel in the figure presents the results for a different state variable. Each panel holds the value of $\hat{\phi}$ and two times its standard error band. The horizontal lines are drawn at a number of key values associated to different null hypotheses, namely: (i) only the long-term component matters $(\phi=0$ or $\phi=\pi)$; (ii) only the short-term component matters $(\phi=\pi / 2)$; (iii) the two scaled components are weighted equally $(\phi=\pi / 4)$; and (iv) the non-decomposed state variable is the relevant state variable $\left(\phi=\arctan \left(\sigma_{S} / \sigma_{L}\right)\right)$.

If we consider the patterns for the $\log$ dividend yield (DY) in Figure 8 , the value of $\phi$ changes gradually across the different filters (as indicated by the value of $x$ on the horizontal axis). Also the standard errors slightly increase for filters that take a longer-term definition of the short-term component up to $x=48$, and then decrease. Intersecting the standard error band with the horizontal lines in the graph indicates that we cannot reject the hypothesis that only the short-term component matters for asset allocation for $\mathrm{CF}(6)$ and $\mathrm{CF}(12)$. We can reject, on the other hand, that only the long-term component is relevant $(\phi=0$ or $\pi$ ), or that both components are equally relevant (whether weighted or unweighted). The same conclusion hold for $\mathrm{CF}(24)$ if we consider a 90 percent confidence interval. The situation is different for $x=36,48,72$. For these filters, we can no longer reject the hypothesis that only the long-term component matters for asset allocation. For all filters, we can reject the hypothesis that the non-decomposed state variable is optimal, as $\phi=\arctan \left(\sigma_{S} / \sigma_{L}\right)$ is outside the standard error bands in all cases considered.

The results for the default spread (DS) as a state variable are similar to those for the $\log$ dividend yield (DY). For $x=6,12,72$, we can reject that only the short-term component matters, while for $x=6,12,24,36$, that only the long-term component matters can be rejected. For the stock market trend (TR), however, we obtain different results. We cannot reject that it is only the short-term component that matters, but nigher can we reject that both the longterm and short-term component are equally important, whether weighted (except for $x=6$, 12 ) or unweighted. The results for the short rate (SR) are very similar to those of the stock market trend.

For term spread (TS), we also have different results. Except for $\mathrm{CF}(6)$, we can not reject 
that only the long-term component matters in asset allocation decisions, nor that the use of undecomposed state variable $\left(\phi=\arctan \left(\sigma_{S} / \sigma_{L}\right)\right)$ suffices. Moreover, for $x=6,12,48,72$, we cannot reject that equal weighting of the short-term and long-term components is optimal. Except for the $\mathrm{CF}$ (6) filter, however, we also can not reject the hypothesis that $\tan (\phi)=\sigma_{S} / \sigma_{L}$, which is equivalent to using the non-decomposed state variable. Finally we find that the standard error bands for dividend growth (DG) are very wide, implying we cannot reject any of the postulated hypotheses on $\phi$ as the band covers the whole interval $[\pi / 4, \pi]$.

Summarizing, based on the recursive CF filter we find for a number of filters that investor should weight short-term and long-term information differently. Also, for most variables the evidence in favor of the short-term component $(\phi=\pi / 2)$ is stronger than for the long-term component ( $\phi=0$ or $\pi$ ). In section refassetalloc:sec5 we investigate the robustness of the latter result for investment horizons longer than one month. We now first turn to the investment implications of the results in Figure 8.

\subsection{Investment strategies}

Using the weight vectors $\beta$ as presented in Section 4.1, we can plot the optimal fractions invested in each of our three asset categories as a function of the index $\beta^{\prime} Z_{t}$. The results are presented in Figures 9 for the $\mathrm{CF}(12)$ filter. To facilitate a comparison between state variables, we standardize $\beta^{\prime} Z_{t}$ by dividing by its standard deviation.

The optimal asset allocations clearly depend on the state variables for all variables considered. For the default spread (DS), the allocation varies from $50 \%$ in stocks, $20 \%$ in bonds and $30 \%$ in cash for low values of the index to $100 \%$ bonds for high values of the index. The Term spread (TS) also exhibits a shift from low-risk assets (100\%cash) to a mix of higher-risk assets (55\%bonds and $45 \%$ stock). We see similar patterns for the dividend growth rate (DG) and a reverse pattern for the short rate $(\mathrm{SR})$.

The patterns seem to be different for the dividend yield (DY) and the stock market trend (TR). In particular, the pattern is non-monotonic. For the dividend yield (DY), low values of the index imply an asset allocation of $100 \%$ in stocks. For middle values of the index, the optimal mix become less risky as 30-40\% stocks and the rest in cash. For high values of the index, $100 \%$ investing in bonds is optimal. The reverse pattern appears for the stock market trend (TR).

The asset allocations from Figure 9 can also be implemented in a backtest. At the start of each month $t$, we use the value of the index $\hat{\beta}^{\prime} Z_{t}$ to determine the asset allocation $x_{t}$ to be 
implemented over the coming month. This is repeated over the entire sample period. For each combination of a filter and state variable, the resulting returns are used to compute monthly expected returns, standard deviations, Sharpe ratios, and expected utilities. The results are presented in Table 7.

The top block of entries in Table 7 presents the results for the original, non-decomposed state variables as a benchmark for comparing the performance of our investment strategies. We can also compare our results with naive strategies such as a portfolio of $100 \%$ stocks, $100 \%$ bonds, or $50 \%$ stocks and $50 \%$ bonds. The Sharpe ratios for these three portfolios over the period April 1963 to June 2011 are 0.077, 0.068, and 0.100 respectively, and are lower than those for the non-decomposed state variable. For the non-decomposed state variables, the results are quite comparable across the different state variables, both in terms of Sharpe ratios and in terms of expected utilities. If we decompose the state variables in their short-term and long-term components, however, we see that particularly for the dividend yield (DY) and to some extent for the stock market trend (TR), the results improve substantially. The magnitude of the improvement varies with the filter that is used. Given the short (one month) horizon of the investor in this example, the best results are achieved by the $\mathrm{CF}(12)$ and $\mathrm{CF}(24)$ filters, which define the short-term component to be up to one or two years. The Sharpe ratios for DY and TR increase by $65 \%$ and $23 \%$ for decompositions with a 'short-term' up to one-year. The average expected utilities also improve by $25 \%$ and $10 \%$ from 0.0057 and 0.0064 to 0.0071 and 0.0070 , respectively.

Except for short rate, we see the advantage of using the decomposed state variable disappear for $\mathrm{CF}(x)$ filters with larger value of $x$. Particularly for the $\mathrm{CF}(72)$ filter, the results are roughly the same as for the state variables without the decomposition. This is not surprising. For shortterm investor, we also expect the valuable signals to have a stricter short-term character.

In order to further test how the investment strategies could be useful, we also perform a true out-of-sample test. For this, we divide our sample into two subsamples. The first subsample covers the period April 1964 till June 2001 and is used to estimate the optimal index coefficient $\beta$ and functional form of $x\left(\beta^{\prime} Z_{t}\right)$. The second subsample covers July 2001 to June 2011 and is used to implement the optimal asset allocation strategy. AT the start of each month in the second subsample, we use the estimated $\beta$ to calculate the value of index. This value is used to compute $x\left(\beta^{\prime} Z_{t}\right)$ using the form of $x(\cdot)$ as estimated on the first subsample. Subsequently, we calculate the return over that month. This is repeated for all months in the second subsample and we calculate the Sharpe ratio and average utility for each state variable and filter.

The results are provided in Table 8. The Sharpe ratios for our 3 naive benchmarks, namely 
the portfolio of $100 \%$ stocks, $100 \%$ bonds, and $50 \%$ stocks and $50 \%$ bonds are $0.040,0.164$, and 0.108 , respectively.

Comparing the out-of-sample Sharpe ratios of different portfolio strategies with the naive benchmarks reveals that particularly for the dividend yield (DY), the improvement in Sharpe ratios is substantial and up to $92 \%$ higher than that for the non-decomposed dividend yield. Also the Sharpe ratios for the stock market trend (TR) increase up to $16 \%$ over that based on the non-decomposed trend. The performance based on the other strategies is typically at par or worse compared to the non-decomposed results. We conclude that particularly the results for the dividend yield and trend are promising and robust in-sample and out-of-sample. The results for the other state variable are not strong, whether the state variable are decomposed or not.

\section{Robustness analysis}

\subsection{Alternative decomposition filters}

So far, we have concentrated our analysis on the Christiano-Fitzgerald filter as the more recent bandpass filter. The Christiano-Fitzgerald filter allowed us to be explicit about our definition of the short-term component. An alternative filter that is very popular and widely used is that of Hodrick and Prescott (1997). We repeat some of our earlier results for this filter to check the robustness of our conclusion that the short-term components of commonly used state variables are most important for asset allocation decisions, particularly for dividend yield and the stock market trend.

To provide in-sample and out-of-sample results using the Hodrick-Prescott filter, we use the same strategy that we used for the Christiano-Fitzgerald filter. We use four different versions of Hodrick-Prescott filter using values $\lambda=\{240,900,3600,14400\}$ for the smoothing parameter $\lambda$.

The optimal index and also the in-sample performance of investment strategies based on different versions of the HP filter are presented in Table 9. We observe that the optimal index coefficients very much resemble those of the Christiano-Fitzgerald filter. For most state variables, the loading of the short-term component is larger than that of the long-term component. The exception is again the term spread. The effect of decomposing the state variables into their long-term and short-term components is good for the Sharpe ratio and expected utilities, as can be seen by comparing the results from Table Table 9 with the non-decomposed results in 
Table 7.

We also provide the out-of-sample performance of investment strategies using the HP filter. The results are presented in Table 10. The out-of-sample performance are similar to those for the CF filter. Most state variable are of limited use for asset allocation. The dividend yield and stock market trend are again notable exceptions. For the trend variable, the decomposition does not add value. For the dividend yield, however, we again corroborate over earlier findings that the long-term and short-term decomposition results in sizable increase in the Sharpe ratio of up to $87 \%$. The same holds for the expected utility, which increases up to $63 \%$.

\subsection{Different level of risk aversion}

In this section, we check the effect of a different risk appetite of investors on optimal investment strategies. So far, we performed our analysis for an investor with constant relative risk aversion (CRRA) utility function and relative risk parameter $\gamma=5$. In this section, we present results for an investor with a $\gamma=1,3,5,10,20$. Table 11 shows the optimal index composition for these different utility specifications. In general, the results are highly robust. Only for the default spread the optimal index for $\gamma=3$ is different. This is due to the existence of two local optima. This result is obtained using a bandwidth with $\lambda_{b}=3$ (see the Appendix for more details on the bandwidth and its effect). As explained in the appendix, for some state variables and parameter combinations, the nonparametric techniques are particularly sensitive to the choice of bandwidth. In this particular setting, for example, the bandwidth appears low. If we increase the bandwidth and use $\lambda_{b}=5$, the optimal angle for $\gamma=3$ is $\phi=2.33$, which is in line with the results for other risk aversion levels. We corroborate that the short-term component is important for asset allocation decisions, also for higher or lower degrees of risk aversion.

Figure 10 compares the asset allocation policies obtained using dividend yield as the state variable and the recursive $\mathrm{CF}(12)$ filter for the decomposition. For $\gamma=1$, the optimal asset allocation is $100 \%$ bonds for low values of the index and $100 \%$ stocks for high value of the index. The switch from one to the other is quite abrupt. As the level of risk aversion increase, we invest more in cash for the middle range of index values. Also the percentage invested in stocks starts to decrease. The high allocations to bonds for high values of the index are very persistent, also for high value of $\gamma$. For low values of the index, however, the fraction invested in cash clearly increasing in $\gamma$.

Table 12 presents the performance of different portfolio strategies using different state vari- 
ables and different level of risk aversion. We observe that Sharpe ratios generally increase in the risk aversion parameter $\gamma$. This is mainly the result of the standard deviation $\sigma_{R}$ decreasing more steeply than the average return $\bar{R}$.

\subsection{Longer investment horizons}

So far, we focused on the results for the one-month investment horizon. In this section we study the effect of applying the conditional investment strategies using a 3-month investment horizon. This is particularly important in our current context, as the short-term component maybe less important for investors with a longer investment horizon.

To implement the strategies, we consider a 3-month buy-and-hold investment horizon. We use 3-month non-overlapping returns for stock and bond returns. We also use the recursive CF filter for the decomposition of state variables. The first 121 months are used as a burn-in period to obtain the first values of the long-term and short-term components. We have a total of 193 non-overlapping investment periods to estimate $\beta$ and $x\left(\beta^{\prime} Z_{t}\right)$. As before, the index $\beta^{\prime} Z_{t}$ is standardized by its time-series standard deviation.

Table 13 shows the optimal index composition for different state variables and filters. Comparing these values with the optimal index values for the one-month investment horizon in Table 6 reveals that for about $2 / 3$ of the cases, the absolute values of the loadings for the long-term component increases while that for the short-term component decreases. The results is intuitively clear. For an investor with a 3-month horizon, the long-term component takes a more prominent role in determining the optimal asset allocation.

Figure 11 compare the optimal asset allocations for different state variable using the recursive $\mathrm{CF}(12)$ filter for the decomposition. If we compare these results with the ones in Figure 9, we observe that for all variables, the percentage invested in bonds deceases and the percentage invested in cash increases. To understand this, we note that the average return on one-month treasury bills over the the period April 1963 to June 2011 is $R f_{1 m}=0.00434$, while the average return on 3-month treasury bills is $R f_{3 m}=0.01781$. This is larger than $3 \times R f_{1 m}=0.01304$. Furthermore, average excess stock and bond returns for a one-month horizon are $r_{1 m}^{s}=0.00352$ and $r_{1 m}^{b}=0.00133$, while for a 3-month horizon they are $r_{3 m}^{s}=0.00664$ and $r_{3 m}^{b}=0.00004$. The latter are lower than $3 \times r_{1 m}^{s}=0.01055$ and $3 \times r_{1 m}^{b}=0.00399$. So the average return on cash increases nonproportionally compared to the average excess return on stocks and bonds. This is a prime reason for the higher cash investments at the 3-month horizon.

Finally, Table 14 present the performance of different portfolio strategies using several state 
variables and CF filters. We observe that the Sharpe ratios generally increase, thought not as $S R_{3 m}=\sqrt{3} \times S R_{1 m}$. We again attribute this effect to the average returns for cash and for the risky assets as discussed earlier.

\section{Conclusions}

We decomposed a number of commonly used state variables for asset allocation into their longterm and short-term components using a variety of filtering techniques. It turned out that for state-variables such as the dividend yield, stock market trend and short rate, the short-term components of state variables take a more important role in asset allocation decisions than the long-term components. The results are comparable over a range of decomposition techniques. The moment conditions used for GMM estimation in our empirical work explicitly account for the presence of short-sale constraints.

We implement the induced investment strategies in a backtesting framework, both in-sample and out-of-sample. We find significant improvements in terms of both Sharpe ratios and expected utilities for state variables such as the dividend yield and stock market trend. The improvements also remain robust in the the out-of-sample exercise.

We conclude that investors can benefit from reacting differently to short-term versus longterm dynamics of state variables. The short-term components appear to contribute most to predictability as well as to improved out-of-sample performance of implied investment strategies. The longer-term dynamics become more important for investors with a longer horizon. Both findings are in line with the partial predictability of returns and the opportunity of investors to exploit this information.

\section{References}

Ait-Sahalia, Y. and M. Brandt (2001). Variable selection for portfolio choice. The Journal of Finance 56(4), $1297-1351$.

Ang, A. and G. Bekaert (2007). Stock return predictability: Is it there? Review of Financial Studies 20(3), 651.

Barberis, N. (2000). Investing for the long run when returns are predictable. The Journal of Finance 55(1), $225-264$.

Baxter, M. and R. King (1999). Measuring business cycles: approximate band-pass filters for economic time series. Review of Economics and Statistics 81(4), 575-593. 
Brandt, M. (1999). Estimating portfolio and consumption choice: A conditional Euler equations approach. The Journal of Finance 54(5), 1609-1645.

Campbell, J. and L. Viceira (1999). Consumption and Portfolio Decisions When Expected Returns are Time Varying. Quarterly Journal of Economics 114(2), 433-495.

Christiano, L. and T. Fitzgerald (2003). The Band Pass Filter. International Economic Review 44(2), 435465.

Cochrane, J. (1999). New Facts in Finance. Economic Perspectives 23(3), 36-38.

Cochrane, J. (2008). The dog that did not bark: A defense of return predictability. Review of Financial Studies 21(4), 1533.

Danthine, J. and M. Girardin (1989). Business cycles in Switzerland: a comparative study. European Economic Review 33(1), 31-50.

Doornik, J. (2007). Object-Oriented Matrix Programming using Ox, 3th edn. London: Timberlake Consultants Press and Oxford: www.doornik.com.

Doornik, J. and M. Ooms (2007). Introduction to Ox: An Object-Oriented Matrix Language. Timberlake Consultants Ltd.

Fama, E. and K. French (1988). Dividend yields and expected stock returns. Journal of Financial Economics 22(1), 3-25.

Fama, E. and K. French (1989). Business conditions and expected returns on stocks and bonds. Journal of Financial Economics 25(1), 23-49.

Handa, P. (2006). Does stock return predictability imply improved asset allocation and performance? evidence from the us stock market (1954-2002). Journal of Business 79(5), 2423-2468.

Hansen, L. (1982). Large sample properties of generalized method of moments estimators. Econometrica: Journal of the Econometric Society, 1029-1054.

Harvey, A. (1990). Forecasting, structural time series models and the Kalman filter. Cambridge Univ Press.

Harvey, A. and A. Jaeger (1993). Detrending, stylized facts and the business cycle. Journal of applied econometrics 8(3), 231-247.

Hodrick, R. and E. Prescott (1997). Postwar US Business Cycles: An Empirical Investigation. Journal of Money, Credit \&3 Banking 29(1).

Kandel, S. and R. Stambaugh (1996). On the predictability of stock returns: An asset-allocation perspective. Journal of Finance 51(2), 385-424.

Keim, D. B. and R. F. Stambaugh (1986). Predicting returns in the stock and bond markets. Journal of Financial Economics 17(2), 357-390.

Leser, C. (1961). A simple method of trend construction. Journal of the Royal Statistical Society. Series B (Methodological) 23(1), 91-107.

Lettau, M. and S. Ludvigson (2005). Expected returns and expected dividend growth. Journal of Financial Economics 76(3), 583-626.

Merton, R. (1971). Optimum consumption and portfolio rules in a continuous-time model. Journal of Economic Theory 3(4), 373-413. 
Razzak, W. (1997). The hodrick-prescott technique: A smoother versus a filter:: An application to new zealand gdp. Economics Letters 57(2), 163-168.

Van Binsbergen, J. and R. Koijen (2010). Predictive regressions: A present-value approach. The Journal of Finance 65(4), 1439-1471.

Wachter, J. and M. Warusawitharana (2009). Predictable returns and asset allocation: Should a skeptical investor time the market? Journal of Econometrics 148(2), 162-178. 

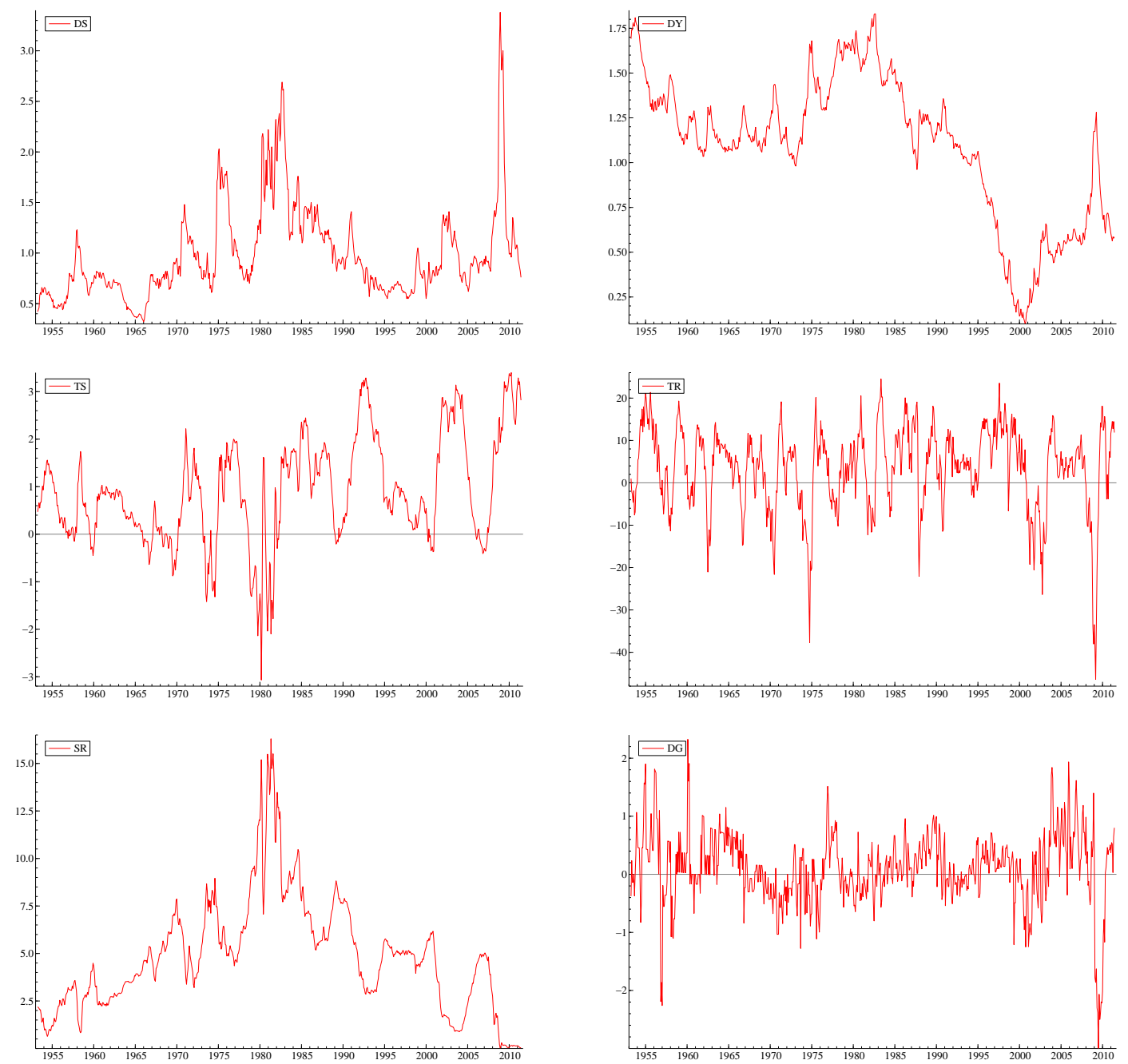

Figure 1: State variables

The panels contain the time-series plots for the different state variables: default spread (DS, upper-left), log dividend-to-price ratio (DY, upper-right), term spread (TS, mid-left), stock market trend (TR, mid-right), short rate (SR, lower-left), dividend growth rate (DG, lower-right). 

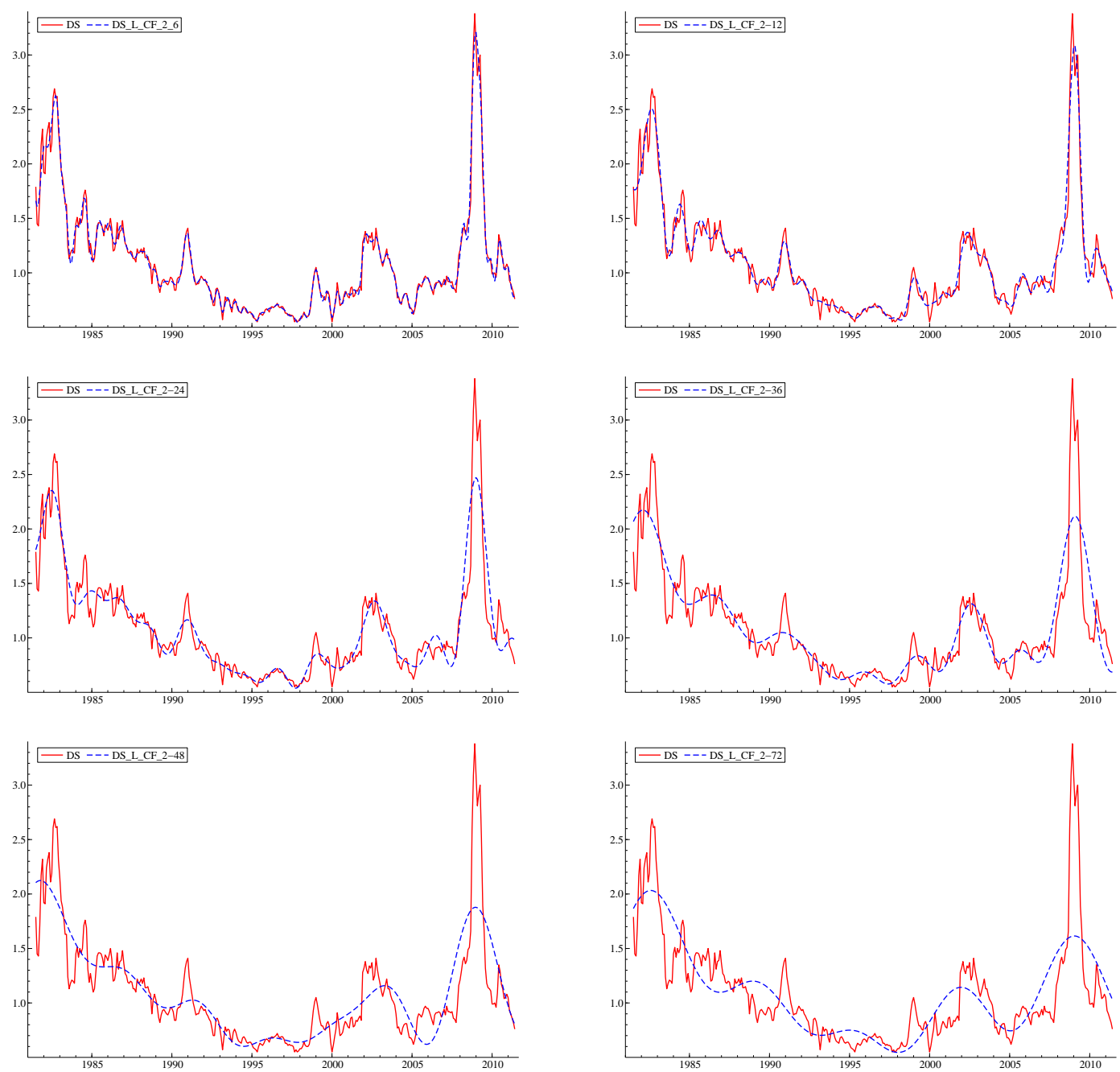

Figure 2: Short-term and long-term components of Default Spread (DS) using the ChristianoFitzgerald filter using different smoothing parameters for last 30 years of data 

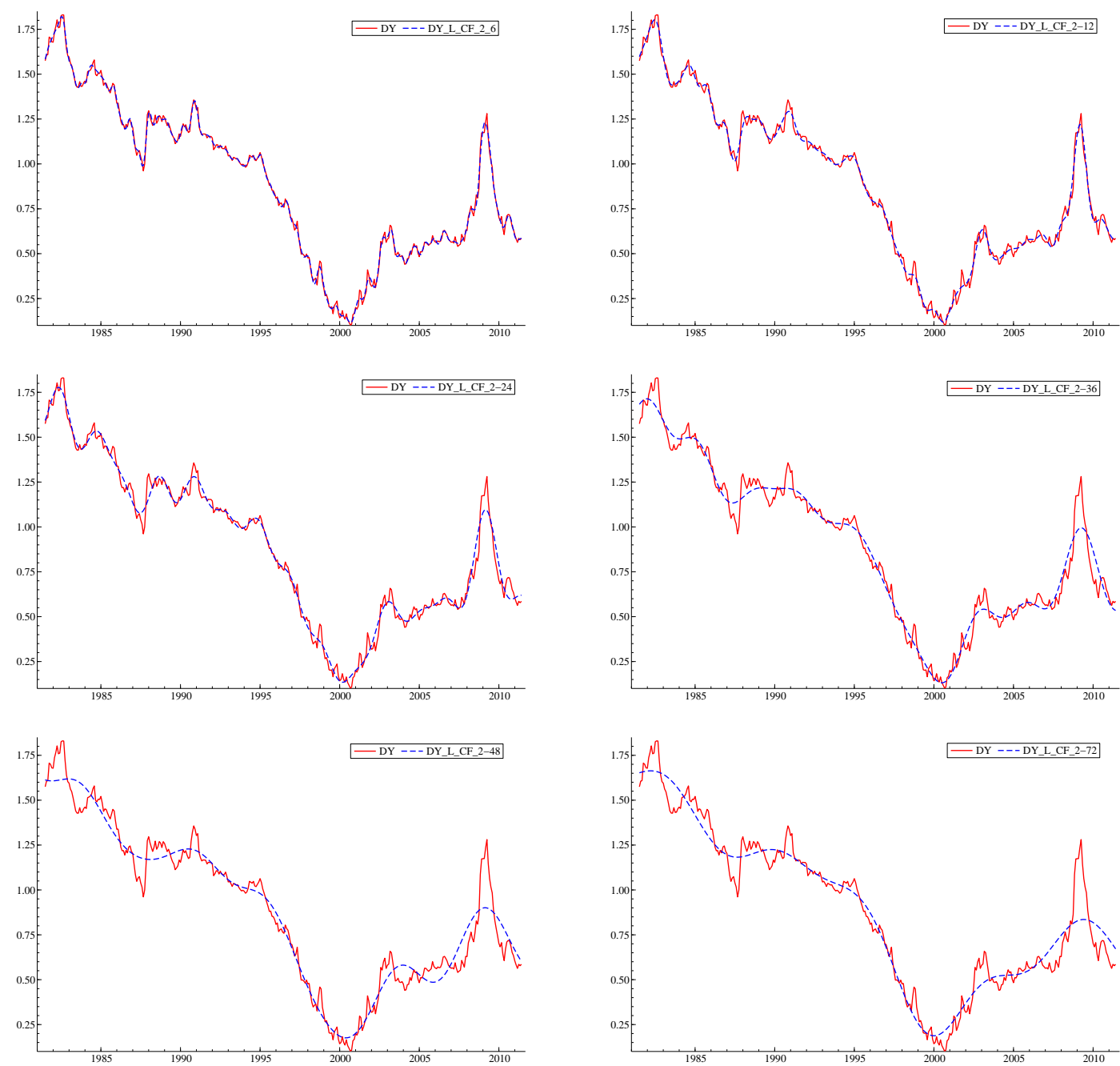

Figure 3: Short-term and long-term components of log Dividend Yield (DY) using the Christiano-Fitzgerald filter using different smoothing parameters for last 30 years of data 

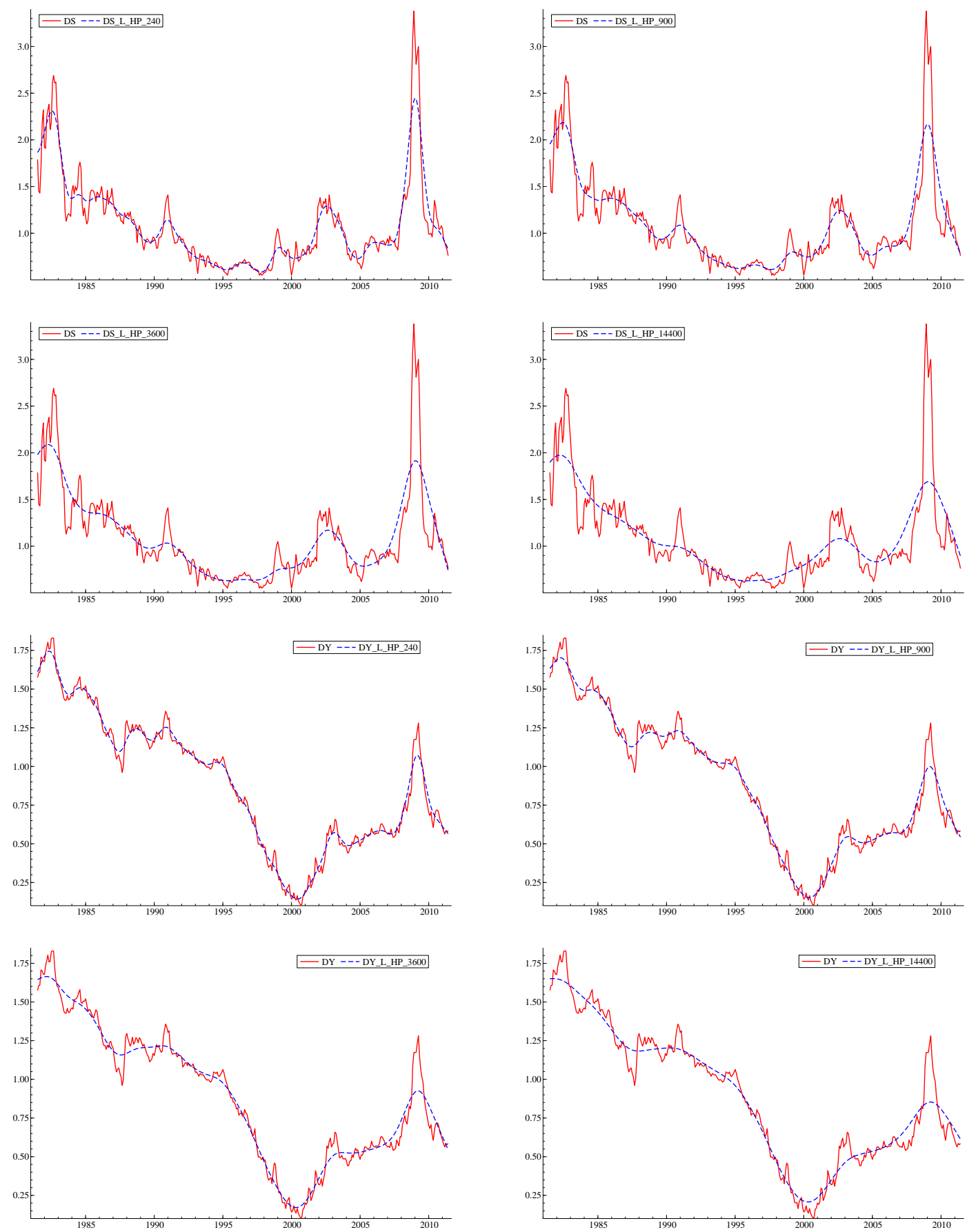

Figure 4: Short-term and long-term components of Default Spread (DS, upper 4 figures) and log Dividend Yield (DY, lower 4 figures) using the the Hodrick-Prescott filter using different smoothing parameters for last 30 years of data 

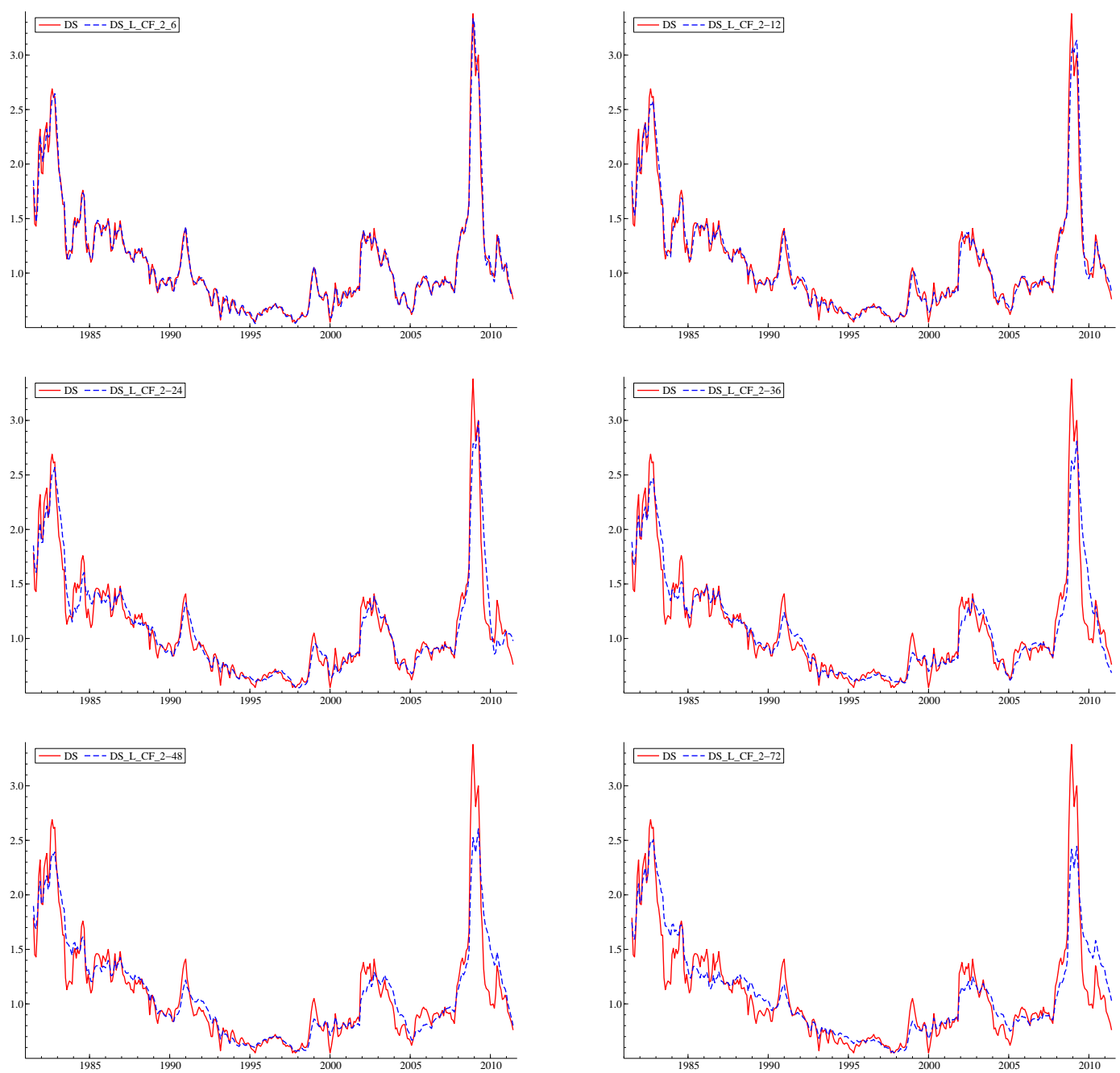

Figure 5: Short-term and long-term components of Default Spread (DS) using recursive Christiano-Fitzgerald filter using different smoothing parameters for last 30 years of data 

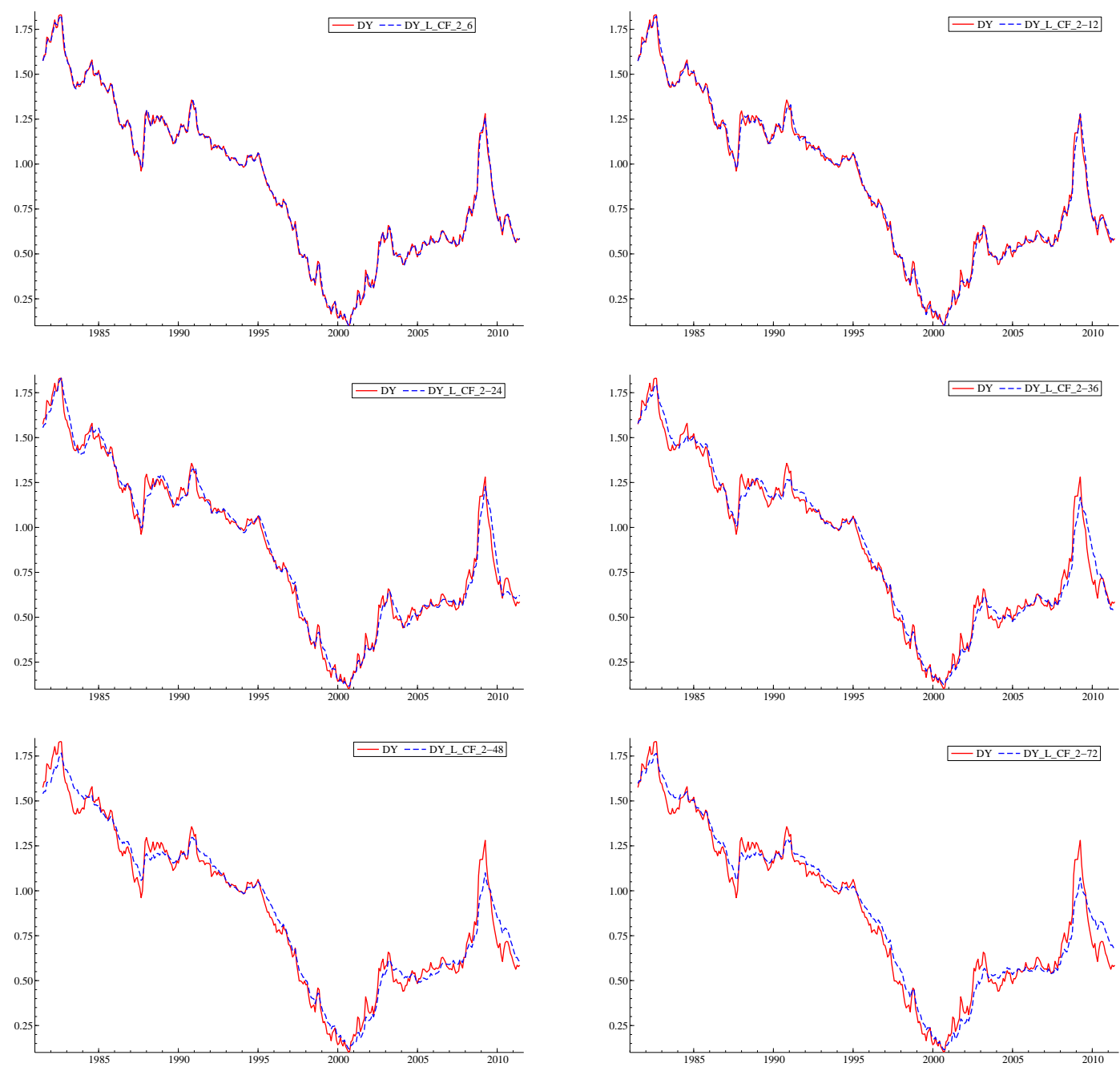

Figure 6: Short-term and long-term components of log Dividend Yield (DY) using recursive Christiano-Fitzgerald filter using different smoothing parameters for last 30 years of data 

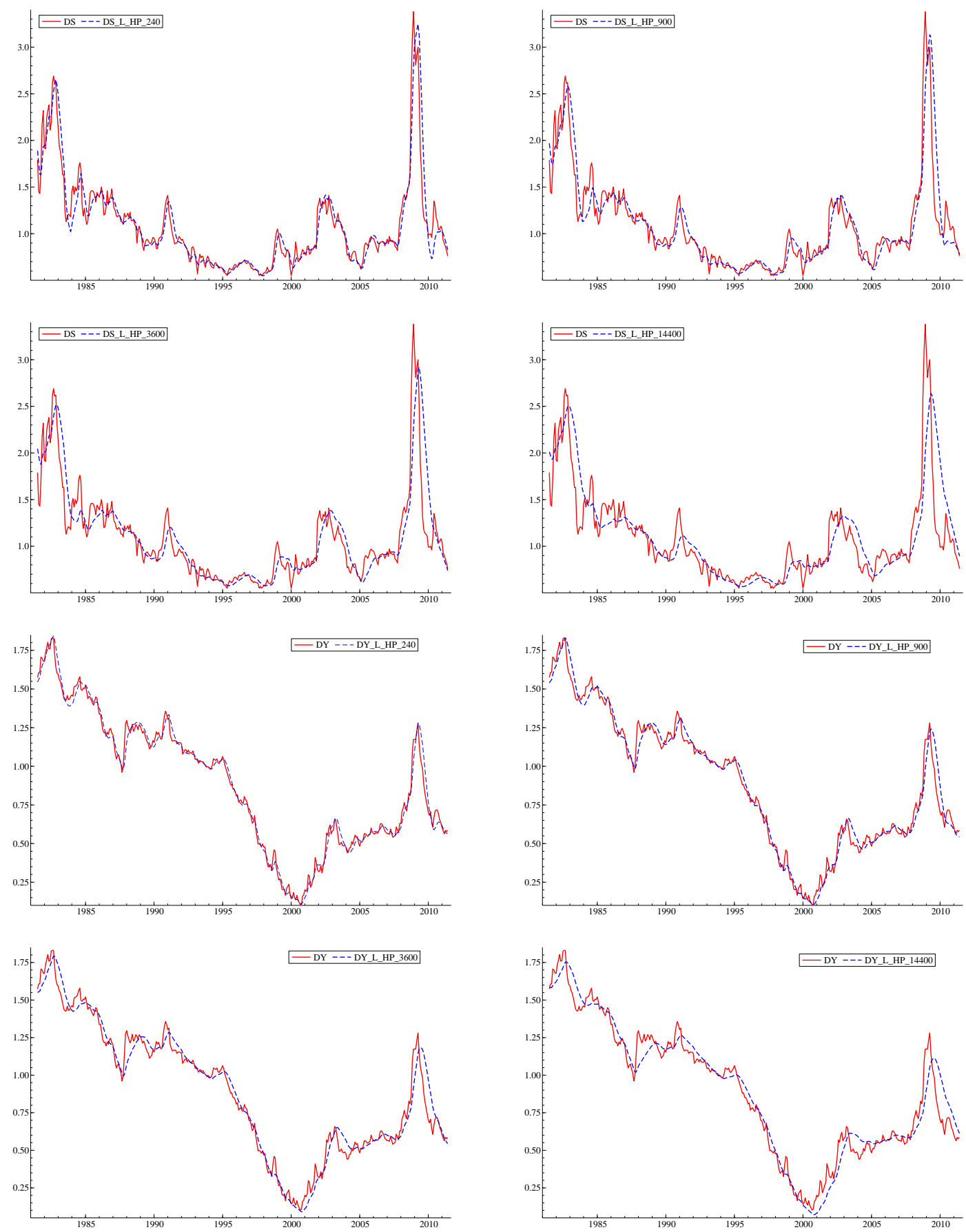

Figure 7: Short-term and long-term components of Default Spread (DS, upper 4 figures) and $\log$ Dividend Yield (DY, lower 4 figures) using recursive Hodrick-Prescott filter using different smoothing parameters for last 30 years of data 
DS

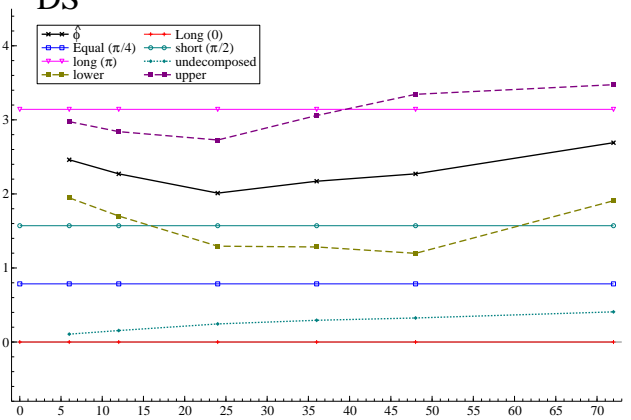

TS
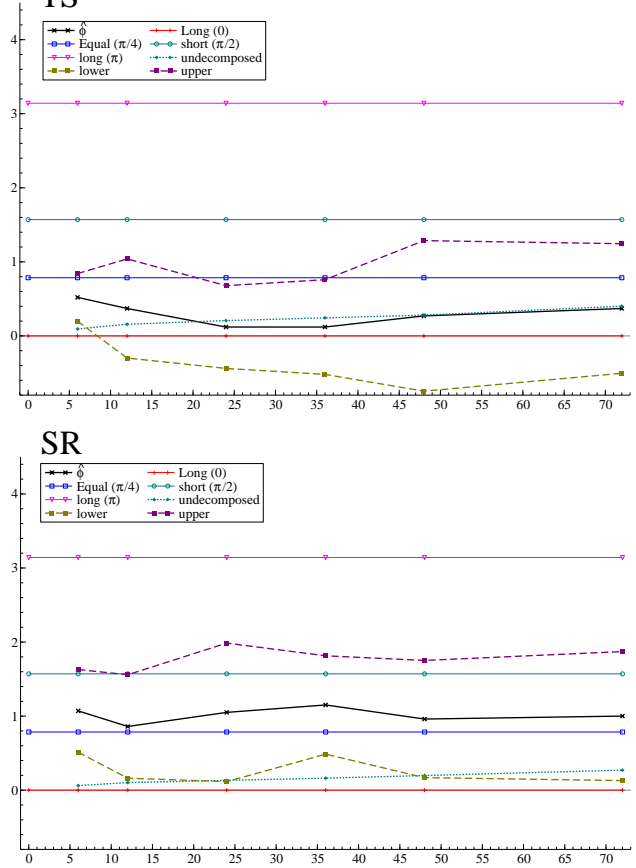

DY

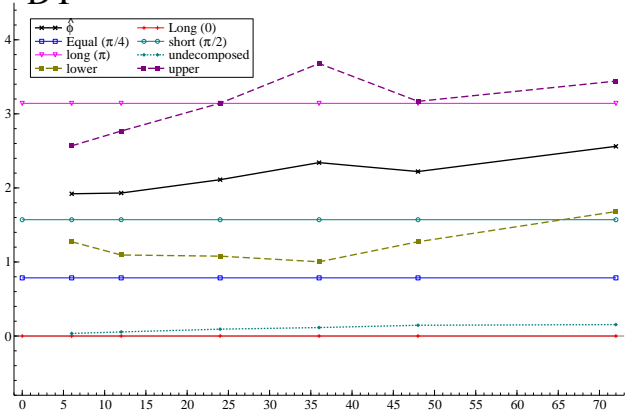

TR

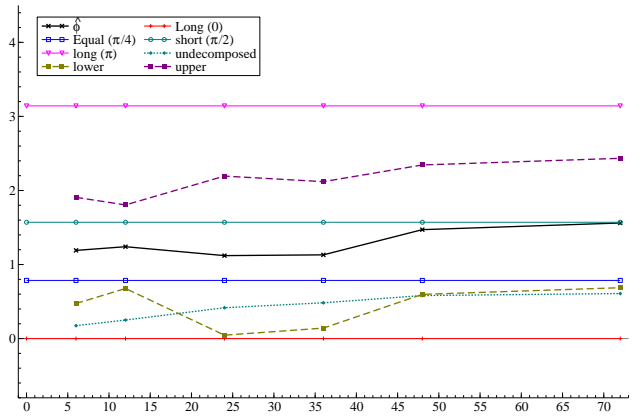

DG

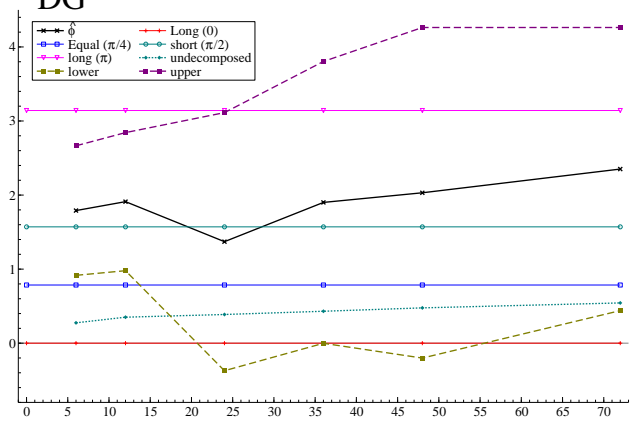

Figure 8: Estimated angle for short-term and long-term components using recursive ChristianoFitzgerald filter

Each graph presents the optimal angle $\phi$ for the long-term and short-term component weights $\beta_{1}=\cos (\phi)$ and $\beta_{2}=\sin (\phi)$, respectively, for different state variables and filters. The horizontal axis gives the value of $x$, where $\mathrm{CF}(x)$ is the recursive Christiano-Fitzgerald filter used for the GMM estimation of $\phi$. The panels are for the different state variables: default spread (DS, upper-left), log dividend-to-price ratio (DY, upperright), term spread (TS, mid-left), stock market trend (TR, mid-right), short rate (SR, lower-left), dividend growth rate (DG, lower-right). Each graph presents the GMM estimate of $\phi$ and two times its standard error band (dashed). The horizontal lines correspond to the different null hypotheses of interest: only the longterm component matters $(\phi=0$ or $\phi=\pi)$, only the short-term component matters $(\phi=\pi / 2)$, both (scaled) components matter equally $(\phi=\pi / 4)$, both (unscaled) components matter equally, such that the original state variable suffices $\left(\tan (\phi)=\sigma_{L} / \sigma_{H}\right)$. 

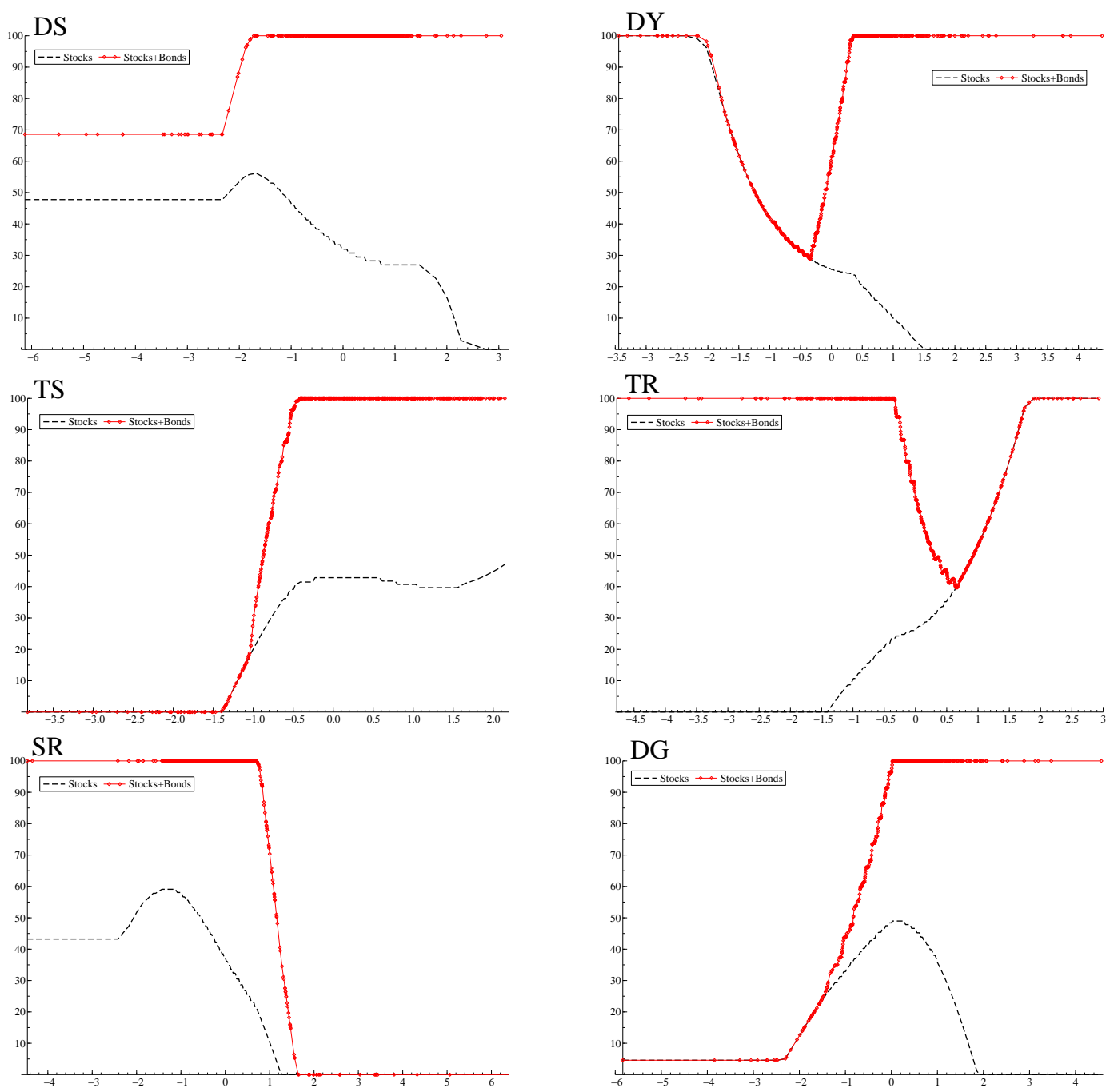

Figure 9: Optimal investment strategies using recursive decomposition and filter $\mathrm{CF}(12)$

Each graph presents the optimal asset allocation as a function of the index $\beta^{\prime} Z_{t}$ that is computed using the optimal $\beta$ from Table 6 and $Z_{t}$ holding the long-term and short-term components of the state variable. The $\mathrm{CF}$ (12) filter is used for the decomposition. Here Christiano-Fitzgerald (CF) filters are run recursively for every month in the sample. For each month $t$, only the past values of state variable are used for estimation of long and short component decomposition so the sample used for decomposition do not contain future values and decomposition for the last value in the sample used as $Z_{t}$. We start with $\mathrm{t}=121$ and in total we have 579 observations for long- and short-term components. The index $\beta^{\prime} Z_{t}$ is standardized by dividing by its timeseries standard deviation. The horizontal axis gives the value of standardized $\beta^{\prime} Z_{t}$. The vertical axis gives the percentage invested in stocks and stocks plus bonds. The panels are for the different state variables: default spread (DS, upper-left), log dividend-to-price ratio (DY, upper-right), term spread (TS, mid-left), stock market trend (TR, mid-right), short rate (SR, lower-left), dividend growth rate (DG, lower-right). 

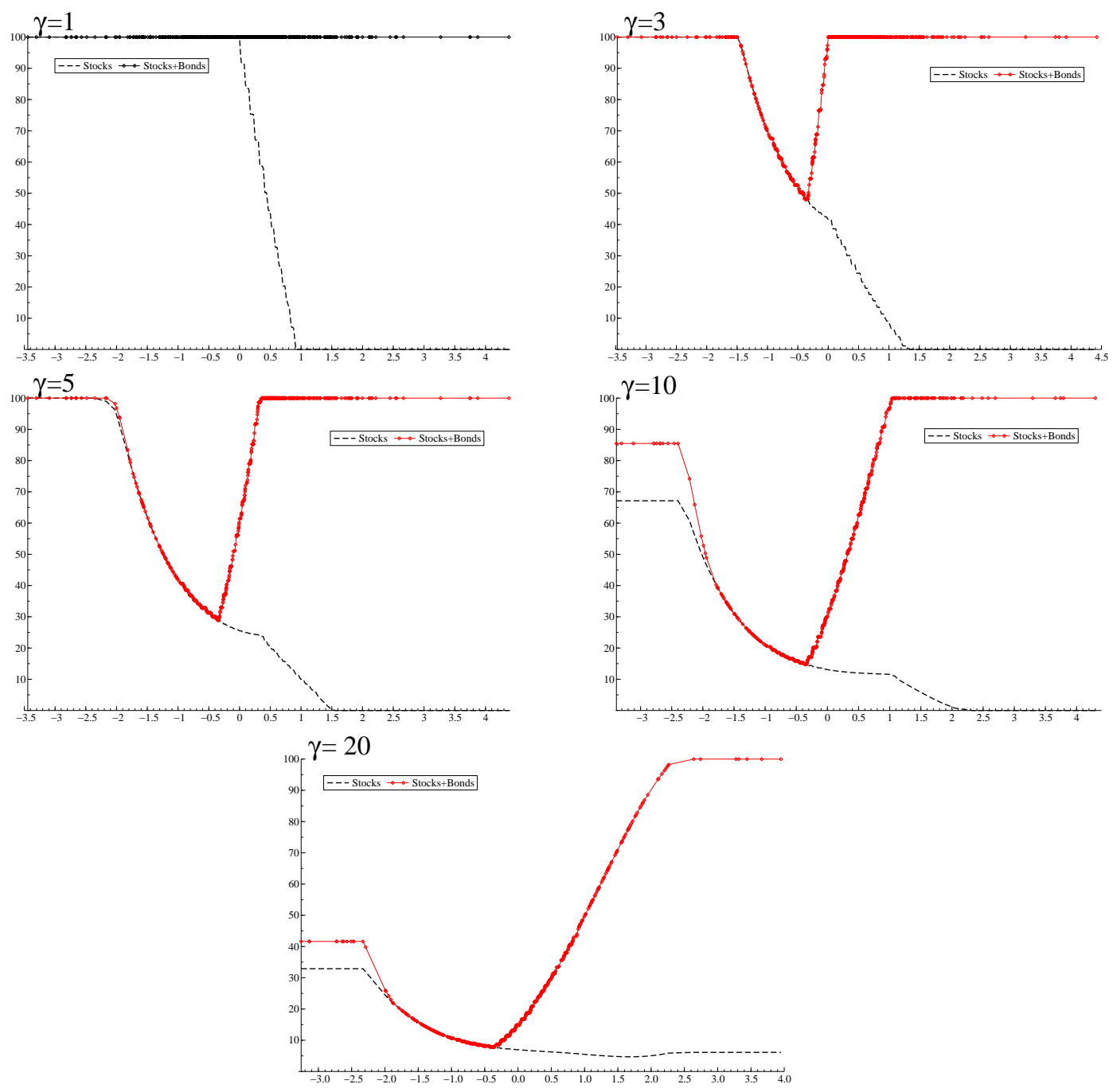

Figure 10: Optimal investment strategies using recursive filtering for dividend yield and filter $\mathrm{CF}(12)$ using CRRA utility function with different risk aversion parameters

Each graph presents the optimal asset allocation as a function of the index $\beta^{\prime} Z_{t}$ that is computed using the optimal $\beta$ from Table 11 and $Z_{t}$ holding the long-term and short-term components of the Dividend yield. The $\mathrm{CF}(12)$ filter is used for the decomposition. The index $\beta^{\prime} Z_{t}$ is standardized by dividing by its time-series standard deviation. The horizontal axis gives the value of standardized $\beta^{\prime} Z_{t}$. The vertical axis gives the percentage invested in stocks and stocks plus bonds. The panels are for CRRA utility function with different investor's relative risk aversion parameter: $\gamma=1$ upper-left, $\gamma=3$ upper-right, $\gamma=5$ mid-left, $\gamma=10$ mid-right and $\gamma=20$ in lower part. 

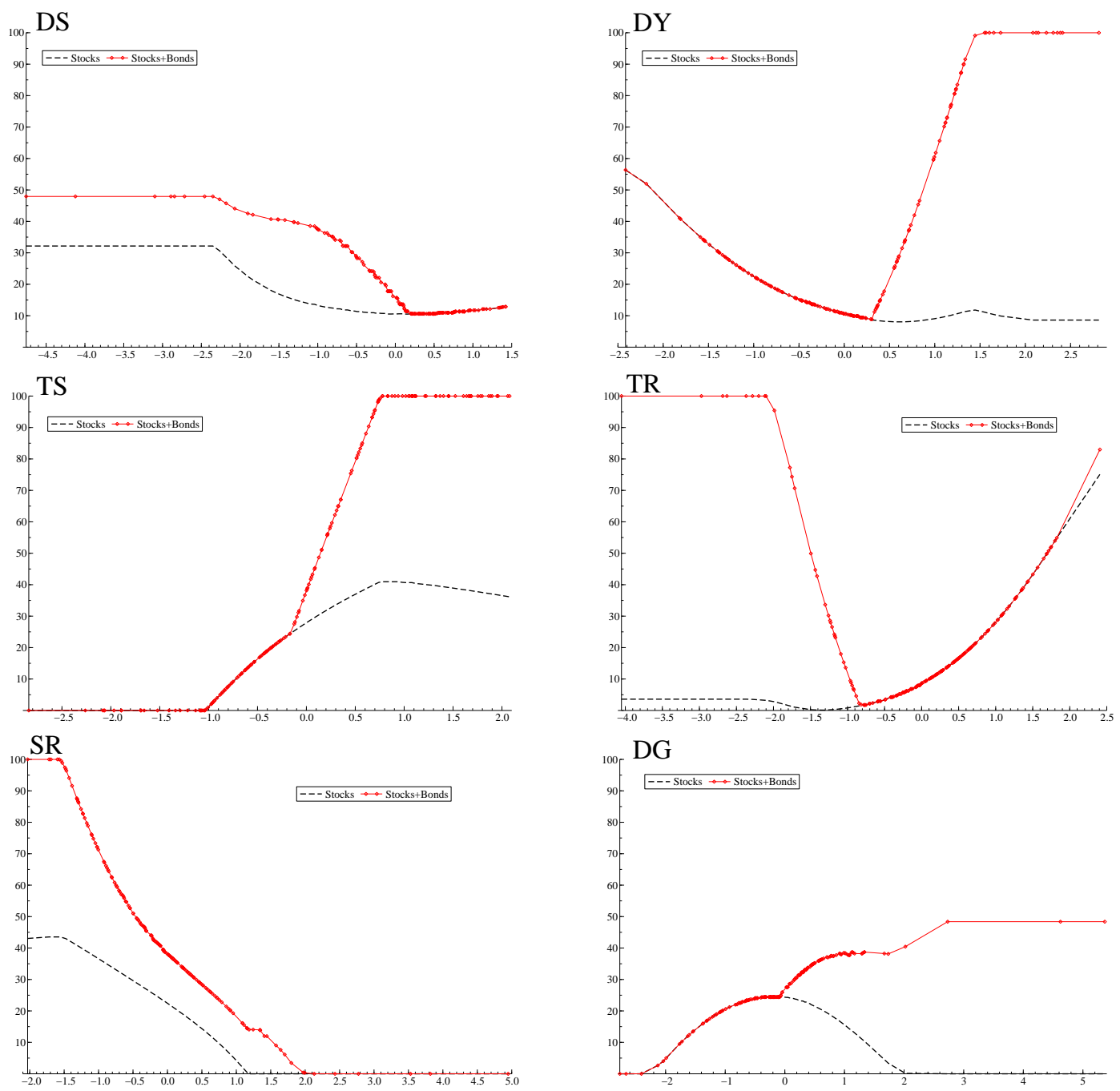

Figure 11: Optimal investment strategies using recursive filtering and 3-month investment horizon for filter $\mathrm{CF}(12)$

Each graph presents the optimal asset allocation as a function of the index $\beta^{\prime} Z_{t}$ that is computed using the optimal $\beta$ from Table 13 and $Z_{t}$ holding the long-term and short-term components of the state variable. Here we use a 3-month buy-and-hold investment horizon, so we use 3-month non-overlapping returns for stock and bond returns. The $\mathrm{CF}(12)$ filter is used for the decomposition. The index $\beta^{\prime} Z_{t}$ is standardized by dividing by its time-series standard deviation. The horizontal axis gives the value of standardized $\beta^{\prime} Z_{t}$. The vertical axis gives the percentage invested in stocks and stocks plus bonds. The panels are for the different state variables: default spread (DS, upper-left), log dividend-to-price ratio (DY, upper-right), term spread (TS, mid-left), stock market trend (TR, mid-right), short rate (SR, lower-left), dividend growth rate (DG, lower-right). 
Table 1: Descriptive statistics

This table shows descriptive statistics of monthly returns on the CRSP stock index, a portfolio of 10-year government bonds, and the one month Treasury bill rate. The panel also includes descriptive statistics of six candidate state variables: the default spread (DS) defined as the difference between the Baa and Aaa yield, the $\log$ dividend-to-price ratio of the $S \& P$ index (DY) with dividends cumulated over the past 12 months, the term spread (TS) defined as the yield difference between 10-year and 1-year government bonds, the stock market trend (TR) defined as the difference of the log index level and its 12 month moving average, short rate (SR), and the (annual) dividend growth rate (DG). The data is sampled monthly from January 1954 to June 2011.

\begin{tabular}{lrrrrrrrrr}
\hline & & & \multicolumn{9}{c}{ Variables } & & & & \\
& Stocks & Bonds & T-bills & DS & DY & TS & TR & SR & DG \\
Mean & & & & & & & & & \\
Median & 1.84 & 0.51 & 0.39 & 0.98 & 1.09 & 0.90 & 3.42 & 4.84 & 0.10 \\
StdDev & 4.39 & 1.85 & 0.24 & 0.46 & 0.40 & 1.10 & 9.88 & 2.93 & 0.63 \\
Skew & -0.81 & 0.52 & 0.92 & 1.80 & -0.56 & 0.09 & -1.07 & 0.94 & -0.69 \\
Kurtosis & 2.93 & 3.85 & 1.43 & 4.37 & -0.36 & -0.08 & 2.23 & 1.49 & 3.37 \\
Min & -25.54 & -8.52 & 0.00 & 0.32 & 0.10 & -3.07 & -46.41 & 0.03 & -2.99 \\
Max & 15.32 & 10.20 & 1.34 & 3.38 & 1.83 & 3.40 & 24.52 & 16.30 & 2.32 \\
& & & & & & & & & \\
Correlation & with: & & & & & & & & \\
Bonds & -0.08 & & & & & & & & \\
T bills & -0.04 & 0.14 & & & & & & & \\
DS & 0.04 & 0.13 & 0.35 & & & & & & \\
DY & 0.09 & 0.09 & 0.40 & 0.28 & & & & & \\
TS & 0.09 & 0.04 & -0.54 & 0.20 & -0.22 & & & & \\
TR & 0.04 & 0.04 & -0.10 & -0.01 & -0.11 & 0.03 & & & \\
SR & -0.05 & 0.12 & 0.98 & 0.34 & 0.42 & -0.56 & 0.01 & & \\
DG & 0.00 & 0.00 & -0.05 & -0.26 & -0.06 & -0.08 & 0.19 & -0.05 & \\
\hline
\end{tabular}


Table 2: Volatilities of the short-term and long-term components using Christiano-Fitzgerald filter

This table shows the time-series standard deviation of the short-term $\left(\sigma_{S}\right)$ and long-term $\left(\sigma_{L}\right)$ components of six state variables: default spread (DS), log dividend-to-price ratio of the $S \& P$ index (DY), term spread (TS), stock market trend (TR), short rate (SR), dividend growth rate (DG). The data is sampled monthly from January 1954 to June 2011. We use the Christiano-Fitzgerald (CF) filter for decomposing each state variable. The cyclical (short-term) component is defined as the component with a cycle up to $x$ months for $x=6,12,24,36,48,72$. The table contains the angle $\phi^{*}=\arctan \left(\sigma_{S} / \sigma_{L}\right)$ which is equivalent to the use of the undecomposed state variable. In this case, $\beta^{\prime} Z_{t}=\cos \left(\phi^{*}\right) Z_{i t}^{L}+\sin \left(\phi^{*}\right) Z_{i t}^{S}=Z_{i t}$ where $Z_{i t}^{L}$ is the standardized (by $\sigma_{L}$ ) long-term component of $Z_{i t}$, and $Z_{i t}^{S}$ is the standardized (by $\sigma_{S}$ ) short-term component.

\begin{tabular}{cccccccc}
\hline \hline & & DS & DY & TS & TR & SR & DG \\
\hline \multirow{2}{*}{$(6)$} & $\sigma_{L}$ & 0.45 & 0.40 & 1.10 & 9.58 & 2.92 & 0.58 \\
& $\sigma_{S}$ & 0.06 & 0.02 & 0.14 & 2.40 & 0.22 & 0.25 \\
& $\phi^{*}$ & 0.13 & 0.05 & 0.12 & 0.25 & 0.08 & 0.41 \\
& & & & & & & \\
$\mathrm{CF}(12)$ & $\sigma_{L}$ & 0.45 & 0.40 & 1.08 & 9.33 & 2.90 & 0.52 \\
& $\sigma_{S}$ & 0.09 & 0.03 & 0.24 & 3.23 & 0.38 & 0.34 \\
& $\phi^{*}$ & 0.20 & 0.07 & 0.22 & 0.33 & 0.13 & 0.58 \\
& & & & & & & \\
$\mathrm{CF}(24)$ & $\sigma_{L}$ & 0.43 & 0.40 & 1.05 & 8.38 & 2.88 & 0.47 \\
& $\sigma_{S}$ & 0.14 & 0.04 & 0.33 & 5.06 & 0.55 & 0.42 \\
& $\phi^{*}$ & 0.32 & 0.11 & 0.31 & 0.54 & 0.19 & 0.73 \\
& & & & & & & \\
$\mathrm{CF}(36)$ & $\sigma_{L}$ & 0.41 & 0.39 & 1.02 & 7.12 & 2.86 & 0.44 \\
& $\sigma_{S}$ & 0.18 & 0.06 & 0.41 & 6.74 & 0.65 & 0.45 \\
& $\phi^{*}$ & 0.42 & 0.15 & 0.38 & 0.76 & 0.22 & 0.80 \\
& & & & & & & \\
$\mathrm{CF}(48)$ & $\sigma_{L}$ & 0.40 & 0.39 & 1.01 & 5.64 & 2.83 & 0.40 \\
& $\sigma_{S}$ & 0.21 & 0.08 & 0.43 & 7.86 & 0.76 & 0.47 \\
& $\phi^{*}$ & 0.48 & 0.20 & 0.41 & 0.95 & 0.26 & 0.87 \\
& & & & & & & \\
$\mathrm{CF}(72)$ & $\sigma_{L}$ & 0.37 & 0.39 & 0.91 & 4.71 & 2.72 & 0.34 \\
& $\sigma_{S}$ & 0.26 & 0.09 & 0.61 & 8.56 & 1.04 & 0.52 \\
& $\phi^{*}$ & 0.62 & 0.24 & 0.59 & 1.07 & 0.37 & 1.00 \\
\hline
\end{tabular}


Table 3: Volatilities of the short-term and long-term components using recursive ChristianoFitzgerald filter

This table are analogous to to Table 2 and shows the time-series standard deviation of the short-term $\left(\sigma_{S}\right)$ and long-term $\left(\sigma_{L}\right)$ components of six state variables. The difference here is that we use a recursive filtering for decomposing state variables to the long and short components; For every month in the period from April 1964 till June 2011, we run the Christiano-Fitzgerald filter using only the data from April 1953 up to the start of the month. The filter produces estimate of the long-term and short-term component at the start of the month. These components are stored in the vector $Z_{t}$ and the process is repeated for all months from April 1964 till June 2011. The state variable are default spread (DS), log dividend-to-price ratio of the $S \& P$ index (DY), term spread (TS), stock market trend (TR), short rate (SR), dividend growth rate (DG). The cyclical (short-term) component is defined as the component with a cycle up to $x$ months for $x=6,12,24,36,48,72$. The table contains the angle $\phi^{*}=\arctan \left(\sigma_{S} / \sigma_{L}\right)$ which is equivalent to the use of the undecomposed state variable. In this case, $\beta^{\prime} Z_{t}=\cos \left(\phi^{*}\right) Z_{i t}^{L}+\sin \left(\phi^{*}\right) Z_{i t}^{S}=Z_{i t}$ where $Z_{i t}^{L}$ is the standardized (by $\sigma_{L}$ ) long-term component of $Z_{i t}$, and $Z_{i t}^{S}$ is the standardized (by $\sigma_{S}$ ) short-term component.

\begin{tabular}{llllllll}
\hline \hline & & DS & DY & TS & TR & SR & DG \\
\hline $\mathrm{CF}(6)$ & $\sigma_{L}$ & 0.47 & 0.41 & 1.18 & 9.84 & 2.94 & 0.58 \\
& $\sigma_{S}$ & 0.05 & 0.01 & 0.11 & 1.74 & 0.18 & 0.16 \\
& $\phi^{*}$ & 0.11 & 0.03 & 0.09 & 0.17 & 0.06 & 0.27 \\
& & & & & & & \\
$\mathrm{CF}(12)$ & $\sigma_{L}$ & 0.47 & 0.41 & 1.17 & 9.76 & 2.93 & 0.55 \\
& $\sigma_{S}$ & 0.07 & 0.02 & 0.19 & 2.50 & 0.30 & 0.20 \\
& $\phi^{*}$ & 0.16 & 0.06 & 0.16 & 0.25 & 0.10 & 0.35 \\
& & & & & & & \\
$\mathrm{CF}(24)$ & $\sigma_{L}$ & 0.46 & 0.41 & 1.15 & 9.43 & 2.91 & 0.53 \\
& $\sigma_{S}$ & 0.11 & 0.04 & 0.24 & 4.17 & 0.39 & 0.21 \\
& $\phi^{*}$ & 0.24 & 0.09 & 0.21 & 0.42 & 0.13 & 0.39 \\
& & & & & & & \\
$\mathrm{CF}(36)$ & $\sigma_{L}$ & 0.45 & 0.41 & 1.13 & 8.56 & 2.89 & 0.50 \\
& $\sigma_{S}$ & 0.14 & 0.05 & 0.28 & 4.49 & 0.48 & 0.23 \\
& $\phi^{*}$ & 0.29 & 0.11 & 0.24 & 0.48 & 0.16 & 0.43 \\
& & & & & & & \\
$\mathrm{CF}(48)$ & $\sigma_{L}$ & 0.44 & 0.41 & 1.14 & 7.74 & 2.89 & 0.49 \\
& $\sigma_{S}$ & 0.15 & 0.06 & 0.33 & 5.08 & 0.58 & 0.25 \\
& $\phi^{*}$ & 0.32 & 0.15 & 0.28 & 0.58 & 0.20 & 0.48 \\
& & & & & & & \\
$\mathrm{CF}(72)$ & $\sigma_{L}$ & 0.42 & 0.40 & 1.08 & 6.94 & 2.81 & 0.45 \\
& $\sigma_{S}$ & 0.18 & 0.06 & 0.46 & 4.82 & 0.78 & 0.27 \\
& $\phi^{*}$ & 0.41 & 0.15 & 0.40 & 0.61 & 0.27 & 0.54 \\
\hline
\end{tabular}


Table 4: Regressions of stock return on long-term and short-term components using recursive Christiano-Fitzgerald filter

This table reports the $R^{2}$ and the partial $R^{2}$ values $R_{L, p}^{2}$ and $R_{S, p}^{2}$ of the long-term and short-term component of state variables, respectively. The partial $R^{2}$ measures the contribution of a variable after all other variables have already been included in the model. The state variables are the default spread (DS), log dividend yield (DY), term spread (TS), stock market trend (TR), short rate (SR), and dividend growth (DG). To decompose each state variable into its long-term and short-term component, we use a recursive Christiano-Fitzgerald filter, where the short-term is defined as up to $x$ months for $x=6,12,24,36,48,72$. The top block of entries gives the results for the non-decomposed state variables. The sample covers April 1963 to June 2011.

\begin{tabular}{clrrrrrr}
\hline \hline & & DS & DY & TS & TR & SR & DG \\
\hline Not decomposed & $R^{2}$ & 0.001 & 0.002 & 0.012 & 0.001 & 0.006 & 0.000 \\
& & & & & & & \\
$\mathrm{CF}(6)$ & $R_{L, p}^{2}$ & 0.001 & 0.002 & 0.010 & 0.001 & 0.005 & 0.001 \\
& $R_{S, p}^{2}$ & 0.000 & 0.000 & 0.012 & 0.002 & 0.022 & 0.005 \\
& $R^{2}$ & 0.001 & 0.002 & 0.022 & 0.003 & 0.027 & 0.006 \\
$\mathrm{CF}(12)$ & & & & & & & \\
& $R_{L, p}^{2}$ & 0.002 & 0.003 & 0.009 & 0.000 & 0.005 & 0.000 \\
& $R_{S, p}^{2}$ & 0.006 & 0.005 & 0.011 & 0.005 & 0.017 & 0.000 \\
& $R^{2}$ & 0.008 & 0.007 & 0.020 & 0.005 & 0.022 & 0.000 \\
$\mathrm{CF}(24)$ & & & & & & & \\
& $R_{L, p}^{2}$ & 0.003 & 0.003 & 0.008 & 0.000 & 0.003 & 0.000 \\
& $R_{S, p}^{2}$ & 0.005 & 0.006 & 0.008 & 0.004 & 0.019 & 0.000 \\
& $R^{2}$ & 0.007 & 0.009 & 0.017 & 0.004 & 0.022 & 0.000 \\
& & & & & & & \\
$\mathrm{CF}(36)$ & $R_{L, p}^{2}$ & 0.003 & 0.003 & 0.008 & 0.000 & 0.003 & 0.000 \\
& $R_{S, p}^{2}$ & 0.002 & 0.003 & 0.006 & 0.003 & 0.020 & 0.000 \\
& $R^{2}$ & 0.004 & 0.006 & 0.015 & 0.003 & 0.023 & 0.000 \\
& & & & & & & \\
$\mathrm{CF}(48)$ & $R_{L, p}^{2}$ & 0.002 & 0.003 & 0.009 & 0.001 & 0.003 & 0.000 \\
& $R_{S, p}^{2}$ & 0.001 & 0.004 & 0.007 & 0.008 & 0.019 & 0.000 \\
& $R^{2}$ & 0.002 & 0.007 & 0.015 & 0.008 & 0.022 & 0.000 \\
& & & & & & & \\
$\mathrm{CF}(72)$ & $R_{L, p}^{2}$ & 0.001 & 0.003 & 0.010 & 0.001 & 0.003 & 0.000 \\
& $R_{S, p}^{2}$ & 0.000 & 0.001 & 0.002 & 0.005 & 0.009 & 0.001 \\
& $R^{2}$ & 0.001 & 0.004 & 0.012 & 0.005 & 0.012 & 0.001 \\
\hline
\end{tabular}


Table 5: Regressions of bond returns on long-term and short-term components using recursive Christiano-Fitzgerald filter

This table reports the $R^{2}$ and the partial $R^{2}$ values resulting from regression of the bond excess return on each state variables as well as on the long-term and short-term component of each state variable. The partial $R^{2}$ (denoted by $R_{L, p}^{2}$ and $R_{S, p}^{2}$ for the long-term and short-term component respectively) measures the contribution of a variable after all other variables have already been included in the model. The state variables are the default spread (DS), log dividend yield (DY), term spread (TS), stock market trend (TR), short rate (SR), and dividend growth (DG). To decompose each state variable into its long-term and short-term component, we use a recursive Christiano-Fitzgerald filter, where the short-term is defined as up to $x$ months for $x=6,12,24,36,48,72$. The top block of entries gives the results for the non-decomposed state variables. The sample covers April 1963 to June 2011.

\begin{tabular}{ccrrrrrr}
\hline \hline & & DS & DY & TS & TR & SR & DG \\
\hline Not decomposed & $R^{2}$ & 0.006 & 0.000 & 0.014 & 0.008 & 0.001 & 0.000 \\
& & & & & & & \\
CF(6) & $R_{L, p}^{2}$ & 0.004 & 0.001 & 0.014 & 0.004 & 0.001 & 0.000 \\
& $R_{S, p}^{2}$ & 0.020 & 0.036 & 0.000 & 0.027 & 0.010 & 0.004 \\
& $R^{2}$ & 0.023 & 0.037 & 0.014 & 0.031 & 0.011 & 0.004 \\
$\mathrm{CF}(12)$ & & & & & & & \\
& $R_{L, p}^{2}$ & 0.003 & 0.001 & 0.014 & 0.004 & 0.001 & 0.002 \\
& $R_{S, p}^{2}$ & 0.021 & 0.021 & 0.000 & 0.021 & 0.004 & 0.009 \\
& $R^{2}$ & 0.023 & 0.022 & 0.014 & 0.024 & 0.004 & 0.010 \\
$\mathrm{CF}(24)$ & & & & & & & \\
& $R_{L, p}^{2}$ & 0.003 & 0.001 & 0.018 & 0.005 & 0.001 & 0.000 \\
& $R_{S, p}^{2}$ & 0.008 & 0.005 & 0.002 & 0.006 & 0.002 & 0.001 \\
& $R^{2}$ & 0.011 & 0.006 & 0.019 & 0.010 & 0.002 & 0.001 \\
$\mathrm{CF}(36)$ & & & & & & & \\
& $R_{L, p}^{2}$ & 0.004 & 0.001 & 0.017 & 0.004 & 0.000 & 0.001 \\
& $R_{S, p}^{2}$ & 0.003 & 0.002 & 0.001 & 0.004 & 0.005 & 0.004 \\
& $R^{2}$ & 0.006 & 0.002 & 0.018 & 0.009 & 0.006 & 0.004 \\
& & & & & & & \\
$\mathrm{CF}(48)$ & $R_{L, p}^{2}$ & 0.004 & 0.001 & 0.016 & 0.004 & 0.000 & 0.000 \\
& $R_{S, p}^{2}$ & 0.002 & 0.005 & 0.000 & 0.003 & 0.005 & 0.001 \\
& $R^{2}$ & 0.006 & 0.006 & 0.016 & 0.008 & 0.005 & 0.002 \\
& & & & & & & \\
$\mathrm{CF}(72)$ & $R_{L, p}^{2}$ & 0.005 & 0.001 & 0.012 & 0.004 & 0.000 & 0.001 \\
& $R_{S, p}^{2}$ & 0.000 & 0.003 & 0.003 & 0.001 & 0.008 & 0.002 \\
& $R^{2}$ & 0.006 & 0.004 & 0.014 & 0.008 & 0.007 & 0.002 \\
\hline
\end{tabular}


Table 6: Optimal index composition using recursive Christiano-Fitzgerald filter

This table presents the GMM estimates of the weights to be used for the long-term and short-term components of a single state variable. The state variables considered are the default spread (DS), log dividend yield (DY), term spread (TS), stock market trend (TR), short rate (SR), and dividend growth rate (DG). We set $\beta_{1}$ and $\beta_{2}$ as the weights of the long-term and short-term components, respectively, with $\beta_{1}=\cos (\phi)$ and $\beta_{2}=\sin (\phi)$, with $\phi \in[0, \pi]$. Standard errors of optimal $\phi$ are provided in parenthesis. We use a recursive ChristianoFitzgerald (CF) filter for decomposing each state variable. The cyclical (short-term) component is defined as the component with a cycle up to $x$ months for $x=6,12,24,36,48,72$.

\begin{tabular}{|c|c|c|c|c|c|c|c|}
\hline & & $\overline{\mathrm{DS}}$ & $\overline{D P Y}$ & TS & TR & $\overline{\text { SR }}$ & $\overline{D G}$ \\
\hline \multirow[t]{3}{*}{$\mathrm{CF}(6)$} & $\hat{\phi}$ & $\begin{array}{c}2.461 \\
(0.262)\end{array}$ & $\begin{array}{c}1.921 \\
(0.330)\end{array}$ & $\begin{array}{c}0.520 \\
(0.164)\end{array}$ & $\begin{array}{l}1.191 \\
(0.366)\end{array}$ & $\begin{array}{c}1.071 \\
(0.285)\end{array}$ & $\begin{array}{c}1.791 \\
(0.446)\end{array}$ \\
\hline & $\beta_{1}$ & -0.777 & -0.343 & 0.868 & 0.371 & 0.480 & -0.218 \\
\hline & $\beta_{2}$ & 0.629 & 0.939 & 0.497 & 0.929 & 0.877 & 0.976 \\
\hline \multirow[t]{3}{*}{$\mathrm{CF}(12)$} & $\hat{\phi}$ & $\begin{array}{c}2.271 \\
(0.291)\end{array}$ & $\begin{array}{l}1.931 \\
(0.427)\end{array}$ & $\begin{array}{c}0.370 \\
(0.341)\end{array}$ & $\begin{array}{l}1.241 \\
(0.288)\end{array}$ & $\begin{array}{c}0.860 \\
(0.356)\end{array}$ & $\begin{array}{l}1.911 \\
(0.476)\end{array}$ \\
\hline & $\beta_{1}$ & -0.644 & -0.352 & 0.932 & 0.324 & 0.652 & -0.334 \\
\hline & $\beta_{2}$ & 0.765 & 0.936 & 0.362 & 0.946 & 0.758 & 0.943 \\
\hline \multirow[t]{3}{*}{$\mathrm{CF}(24)$} & $\hat{\phi}$ & $\begin{array}{c}2.011 \\
(0.366)\end{array}$ & $\begin{array}{c}2.111 \\
(0.527)\end{array}$ & $\begin{array}{c}0.120 \\
(0.285)\end{array}$ & $\begin{array}{l}1.121 \\
(0.548)\end{array}$ & $\begin{array}{c}1.051 \\
(0.477)\end{array}$ & $\begin{array}{c}1.371 \\
(0.889)\end{array}$ \\
\hline & $\beta_{1}$ & -0.426 & -0.514 & 0.993 & 0.435 & 0.497 & 0.199 \\
\hline & $\beta_{2}$ & 0.905 & 0.858 & 0.120 & 0.900 & 0.868 & 0.980 \\
\hline \multirow[t]{3}{*}{$\mathrm{CF}(36)$} & $\hat{\phi}$ & $\begin{array}{c}2.171 \\
(0.453)\end{array}$ & $\begin{array}{c}2.341 \\
(0.683)\end{array}$ & $\begin{array}{c}0.120 \\
(0.326)\end{array}$ & $\begin{array}{c}1.131 \\
(0.504)\end{array}$ & $\begin{array}{c}1.151 \\
(0.339)\end{array}$ & $\begin{array}{c}1.901 \\
(0.971)\end{array}$ \\
\hline & $\beta_{1}$ & -0.565 & -0.696 & 0.993 & 0.426 & 0.408 & -0.324 \\
\hline & $\beta_{2}$ & 0.825 & 0.718 & 0.120 & 0.905 & 0.913 & 0.946 \\
\hline \multirow[t]{3}{*}{$\mathrm{CF}(48)$} & $\hat{\phi}$ & $\begin{array}{c}2.271 \\
(0.548)\end{array}$ & $\begin{array}{c}2.221 \\
(0.484)\end{array}$ & $\begin{array}{c}0.270 \\
(0.519)\end{array}$ & $\begin{array}{c}1.471 \\
(0.446)\end{array}$ & $\begin{array}{c}0.960 \\
(0.404)\end{array}$ & $\begin{array}{c}2.031 \\
(1.139)\end{array}$ \\
\hline & $\beta_{1}$ & -0.644 & -0.605 & 0.964 & 0.100 & 0.573 & -0.444 \\
\hline & $\beta_{2}$ & 0.765 & 0.796 & 0.267 & 0.995 & 0.819 & 0.896 \\
\hline \multirow[t]{3}{*}{$\mathrm{CF}(72)$} & $\hat{\phi}$ & $\begin{array}{c}2.691 \\
(0.400)\end{array}$ & $\begin{array}{c}2.561 \\
(0.449)\end{array}$ & $\begin{array}{c}0.370 \\
(0.446)\end{array}$ & $\begin{array}{l}1.561 \\
(0.446)\end{array}$ & $\begin{array}{c}1.001 \\
(0.444)\end{array}$ & $\begin{array}{c}2.351 \\
(0.975)\end{array}$ \\
\hline & $\beta_{1}$ & -0.900 & -0.836 & 0.932 & 0.010 & 0.540 & -0.704 \\
\hline & $\beta_{2}$ & 0.435 & 0.548 & 0.362 & 1.000 & 0.842 & 0.711 \\
\hline
\end{tabular}


Table 7: In-sample performance of portfolio strategies using recursive Christiano-Fitzgerald filter

This table shows the in-sample performance of portfolio strategies based on different filter and state variable pairs. At the start of each month, the index $\hat{\beta}^{\prime} Z_{t}$ is computed with the $\beta$ from Table 6 and the $Z_{t}$ holding the long-term and short-term component of the state variable. The index is used to compute the optimal (conditional) asset allocation, which is implemented over the coming month. This procedure is repeated for all months in the sample. The returns are stored and used to compute average returns $(\bar{R})$, standard deviations $(\sigma)$, Sharpe ratios (Sharpe), and expected utilities $(\bar{U})$. As state variables, we consider the default spread (DS), log dividend yield (DY), term spread (TS), stock market trend (TR), short rate (SR), and dividend growth (DG). To decompose each state variable into its long-term and short-term component, we use a recursive ChristianoFitzgerald filter, where the short-term is defined as up to $x$ months for $x=6,12,24,36,48,72$. The top block of entries gives the results for the non-decomposed state variables. The sample covers April 1963 to June 2011. The weights used for $\beta^{\prime} Z_{t}$ are those of the recursive decomposition from Table 6.

\begin{tabular}{|c|c|c|c|c|c|c|c|}
\hline & & $\overline{\mathrm{DS}}$ & $\overline{\mathrm{DY}}$ & TS & TR & $\overline{\text { SR }}$ & $\overline{\mathrm{DG}}$ \\
\hline \multirow[t]{4}{*}{ Not decomposed } & $\bar{R}$ & 0.002 & 0.002 & 0.003 & 0.004 & 0.003 & 0.002 \\
\hline & $\sigma_{R}$ & 0.019 & 0.018 & 0.019 & 0.023 & 0.019 & 0.017 \\
\hline & Sharpe & 0.108 & 0.125 & 0.169 & 0.157 & 0.140 & 0.126 \\
\hline & $\hat{U}$ & 0.0054 & 0.0057 & 0.0065 & 0.0064 & 0.0060 & 0.0057 \\
\hline \multirow[t]{4}{*}{$\mathrm{CF}(6)$} & $\bar{R}$ & 0.002 & 0.003 & 0.003 & 0.003 & 0.003 & 0.002 \\
\hline & $\sigma_{R}$ & 0.021 & 0.017 & 0.019 & 0.019 & 0.019 & 0.017 \\
\hline & Sharpe & 0.114 & 0.185 & 0.168 & 0.183 & 0.167 & 0.132 \\
\hline & $\hat{U}$ & 0.0056 & 0.0067 & 0.0065 & 0.0068 & 0.0065 & 0.0058 \\
\hline \multirow[t]{4}{*}{$\mathrm{CF}(12)$} & $\bar{R}$ & 0.003 & 0.004 & 0.003 & 0.004 & 0.003 & 0.003 \\
\hline & $\sigma_{R}$ & 0.020 & 0.018 & 0.019 & 0.018 & 0.021 & 0.020 \\
\hline & Sharpe & 0.129 & 0.206 & 0.168 & 0.193 & 0.157 & 0.145 \\
\hline & $\hat{U}$ & 0.0058 & 0.0071 & 0.0065 & 0.0070 & 0.0064 & 0.0061 \\
\hline \multirow[t]{4}{*}{$\mathrm{CF}(24)$} & $\bar{R}$ & 0.003 & 0.004 & 0.003 & 0.004 & 0.003 & 0.002 \\
\hline & $\sigma_{R}$ & 0.019 & 0.021 & 0.019 & 0.021 & 0.020 & 0.017 \\
\hline & Sharpe & 0.135 & 0.179 & 0.172 & 0.167 & 0.177 & 0.125 \\
\hline & $\hat{U}$ & 0.0059 & 0.0069 & 0.0066 & 0.0067 & 0.0067 & 0.0057 \\
\hline \multirow[t]{4}{*}{$\mathrm{CF}(36)$} & $\bar{R}$ & 0.002 & 0.003 & 0.003 & 0.003 & 0.003 & 0.002 \\
\hline & $\sigma_{R}$ & 0.017 & 0.021 & 0.019 & 0.020 & 0.019 & 0.019 \\
\hline & Sharpe & 0.133 & 0.152 & 0.173 & 0.148 & 0.171 & 0.115 \\
\hline & $\hat{U}$ & 0.0058 & 0.0063 & 0.0066 & 0.0062 & 0.0066 & 0.0055 \\
\hline \multirow[t]{4}{*}{$\mathrm{CF}(48)$} & $\bar{R}$ & 0.002 & 0.003 & 0.003 & 0.003 & 0.004 & 0.002 \\
\hline & $\sigma_{R}$ & 0.019 & 0.022 & 0.019 & 0.021 & 0.021 & 0.019 \\
\hline & Sharpe & 0.118 & 0.155 & 0.169 & 0.156 & 0.172 & 0.118 \\
\hline & $\hat{U}$ & 0.0056 & 0.0064 & 0.0065 & 0.0064 & 0.0067 & 0.0056 \\
\hline \multirow[t]{4}{*}{$\mathrm{CF}(72)$} & $\bar{R}$ & 0.002 & 0.003 & 0.003 & 0.003 & 0.004 & 0.002 \\
\hline & $\sigma_{R}$ & 0.018 & 0.022 & 0.019 & 0.021 & 0.022 & 0.019 \\
\hline & Sharpe & 0.119 & 0.140 & 0.169 & 0.154 & 0.181 & 0.120 \\
\hline & $\hat{U}$ & 0.0056 & 0.0061 & 0.0065 & 0.0064 & 0.0070 & 0.0056 \\
\hline
\end{tabular}


Table 8: Out-of-sample performance of portfolio strategies using recursive Christiano-Fitzgerald filter

The table presents out-of-sample performance of different portfolio strategies using recursive filtering. For the period April 1963 to June 2011, the Christiano-Fitzgerald (CF) filters are run recursively for every month in the sample and only the last decomposition of these filters is used in $Z_{t}$. Then we divide this sample to two sub-samples. Period April 1963 to June 2001 used for estimating optimal index composition and optimal portfolio rules for each value of index realized at start of each month in this period. Then using this optimal index combination, we construct the value of index for the state variables realized at the start of each month in the period July 2001 to June 2011. Then we implement the same (or similar) portfolio rules estimated for the value of index in the in-sample period (1963-04 to 2001-06), for the realized value of index at the start of each month in the later period (2001-07 to 2011-06) and calculate the corresponding portfolio return over the coming month. This procedure is repeated for all months in the later sample. The returns are stored and used to compute average returns $(\bar{R})$, standard deviations $(\sigma)$, Sharpe ratios (Sharpe), and expected utilities $(\bar{U})$. We use the same state variables and the same filters that we used in the Table 7 .

\begin{tabular}{|c|c|c|c|c|c|c|c|}
\hline & & $\overline{\mathrm{DS}}$ & DY & TS & TR & SR & $\overline{\mathrm{DG}}$ \\
\hline \multirow[t]{4}{*}{ Not decomposed } & $\bar{R}$ & 0.000 & 0.003 & 0.001 & 0.005 & 0.002 & 0.002 \\
\hline & $\sigma_{R}$ & 0.027 & 0.020 & 0.029 & 0.020 & 0.038 & 0.022 \\
\hline & Sharpe & 0.002 & 0.151 & 0.046 & 0.239 & 0.052 & 0.078 \\
\hline & $\hat{U}$ & -0.0005 & 0.0035 & 0.0008 & 0.0053 & -0.0003 & 0.0021 \\
\hline \multirow[t]{4}{*}{$\mathrm{CF}(6)$} & $\bar{R}$ & 0.001 & 0.004 & 0.002 & 0.004 & 0.002 & 0.001 \\
\hline & $\sigma_{R}$ & 0.022 & 0.019 & 0.027 & 0.018 & 0.031 & 0.016 \\
\hline & Sharpe & 0.027 & 0.218 & 0.059 & 0.242 & 0.071 & 0.076 \\
\hline & $\hat{U}$ & 0.0008 & 0.0048 & 0.0013 & 0.0051 & 0.0013 & 0.0021 \\
\hline \multirow[t]{4}{*}{$\mathrm{CF}(12)$} & $\bar{R}$ & 0.000 & 0.005 & 0.002 & 0.004 & 0.002 & 0.002 \\
\hline & $\sigma_{R}$ & 0.021 & 0.017 & 0.030 & 0.016 & 0.044 & 0.018 \\
\hline & Sharpe & -0.023 & 0.290 & 0.056 & 0.277 & 0.050 & 0.119 \\
\hline & $\hat{U}$ & -0.0002 & 0.0058 & 0.0009 & 0.0054 & -0.0016 & 0.0029 \\
\hline \multirow[t]{4}{*}{$\mathrm{CF}(24)$} & $\bar{R}$ & -0.001 & 0.005 & 0.001 & 0.004 & 0.002 & 0.001 \\
\hline & $\sigma_{R}$ & 0.023 & 0.017 & 0.027 & 0.017 & 0.046 & 0.027 \\
\hline & Sharpe & -0.023 & 0.272 & 0.048 & 0.233 & 0.036 & 0.033 \\
\hline & $\hat{U}$ & -0.0005 & 0.0054 & 0.0010 & 0.0049 & -0.0027 & 0.0006 \\
\hline \multirow[t]{4}{*}{$\mathrm{CF}(36)$} & $\bar{R}$ & -0.001 & 0.003 & 0.001 & 0.004 & 0.000 & 0.002 \\
\hline & $\sigma_{R}$ & 0.024 & 0.017 & 0.027 & 0.018 & 0.038 & 0.028 \\
\hline & Sharpe & -0.034 & 0.195 & 0.046 & 0.210 & 0.003 & 0.060 \\
\hline & $\hat{U}$ & -0.0008 & 0.0041 & 0.0010 & 0.0045 & -0.0025 & 0.0013 \\
\hline \multirow[t]{4}{*}{$\mathrm{CF}(48)$} & $\bar{R}$ & -0.001 & 0.005 & 0.001 & 0.004 & -0.001 & 0.001 \\
\hline & $\sigma_{R}$ & 0.030 & 0.020 & 0.029 & 0.020 & 0.042 & 0.028 \\
\hline & Sharpe & -0.039 & 0.246 & 0.046 & 0.216 & -0.021 & 0.051 \\
\hline & $\hat{U}$ & -0.0026 & 0.0055 & 0.0008 & 0.0049 & -0.0045 & 0.0010 \\
\hline \multirow[t]{4}{*}{$\mathrm{CF}(72)$} & $\bar{R}$ & -0.002 & 0.005 & 0.001 & 0.005 & -0.001 & 0.001 \\
\hline & $\sigma_{R}$ & 0.032 & 0.017 & 0.029 & 0.024 & 0.043 & 0.022 \\
\hline & Sharpe & -0.077 & 0.285 & 0.050 & 0.193 & -0.022 & 0.033 \\
\hline & $\hat{U}$ & -0.0041 & 0.0056 & 0.0008 & 0.0047 & -0.0047 & 0.0011 \\
\hline
\end{tabular}


Table 9: Optimal Index estimation and in-sample performance of portfolio strategies using recursive Hodrick-Prescott filter

This table shows the in-sample estimation and performance of portfolio strategies based on different HodrickPrescott filters and state variable pairs. We set $\beta_{1}$ and $\beta_{2}$ as the weights of the long-term and short-term components, respectively, with $\beta_{1}=\cos (\phi)$ and $\beta_{2}=\sin (\phi)$, with $\phi \in[0, \pi]$. At the start of each month, the index $\hat{\beta}^{\prime} Z_{t}$ is computed with the estimated optimal vector $\beta$ and the $Z_{t}$ holding the long-term and short-term component of the state variable. The index is used to compute the optimal (conditional) asset allocation, which is implemented over the coming month. This procedure is repeated for all months in the sample. The returns are stored and used to compute average returns $(\bar{R})$, standard deviations $(\sigma)$, Sharpe ratios (Sharpe), and expected utilities $(\bar{U})$. As state variables, we consider the default spread (DS), log dividend yield (DY), term spread (TS), stock market trend (TR), short rate (SR), and dividend growth (DG). To decompose each state variable into its long-term and short-term component, we use the Hodrick-Prescott filter recursively, with smoothing parameters $\lambda, \lambda=240,900,3600,14400$. The sample covers April 1963 to June 2011.

\begin{tabular}{|c|c|c|c|c|c|c|c|}
\hline & & $\overline{\mathrm{DS}}$ & DY & $\overline{T S}$ & TR & SR & $\overline{\mathrm{DG}}$ \\
\hline \multirow[t]{8}{*}{$\mathrm{HP}(240)$} & $\hat{\phi}$ & 1.991 & 2.011 & 0.480 & 1.181 & 0.910 & 1.371 \\
\hline & & $(0.419)$ & $(0.531)$ & $(0.388)$ & $(0.293)$ & $(0.357)$ & $(0.614)$ \\
\hline & $\beta_{1}$ & -0.408 & -0.426 & 0.887 & 0.380 & 0.613 & 0.199 \\
\hline & $\beta_{2}$ & 0.913 & 0.905 & 0.462 & 0.925 & 0.790 & 0.980 \\
\hline & $\bar{R}$ & 0.003 & 0.003 & 0.003 & 0.003 & 0.003 & 0.002 \\
\hline & $\sigma_{R}$ & 0.020 & 0.018 & 0.018 & 0.018 & 0.020 & 0.018 \\
\hline & Sharpe & 0.132 & 0.184 & 0.179 & 0.185 & 0.176 & 0.125 \\
\hline & $\hat{U}$ & 0.0059 & 0.0068 & 0.0067 & 0.0068 & 0.0067 & 0.0057 \\
\hline \multirow[t]{8}{*}{$\mathrm{HP}(900)$} & $\hat{\phi}$ & 2.081 & 2.061 & 0.300 & 1.231 & 0.960 & 1.721 \\
\hline & & $(0.471)$ & $(0.191)$ & $(0.337)$ & $(0.304)$ & $(0.355)$ & (1.153) \\
\hline & $\beta_{1}$ & -0.488 & -0.471 & 0.955 & 0.334 & 0.573 & -0.150 \\
\hline & $\beta_{2}$ & 0.873 & 0.882 & 0.296 & 0.943 & 0.819 & 0.989 \\
\hline & $\bar{R}$ & 0.003 & 0.004 & 0.003 & 0.003 & 0.004 & 0.002 \\
\hline & $\sigma_{R}$ & 0.019 & 0.020 & 0.019 & 0.018 & 0.020 & 0.018 \\
\hline & Sharpe & 0.135 & 0.181 & 0.169 & 0.173 & 0.179 & 0.118 \\
\hline & $\hat{U}$ & 0.0060 & 0.0069 & 0.0065 & 0.0066 & 0.0068 & 0.0056 \\
\hline \multirow[t]{8}{*}{$\operatorname{HP}(3600)$} & $\hat{\phi}$ & 2.651 & 2.091 & 0.360 & 1.191 & 0.980 & 1.831 \\
\hline & & $(0.486)$ & $(0.648)$ & $(0.336)$ & $(0.267)$ & $(0.363)$ & $(2.285)$ \\
\hline & $\beta_{1}$ & -0.882 & -0.497 & 0.936 & 0.371 & 0.557 & -0.257 \\
\hline & $\beta_{2}$ & 0.471 & 0.868 & 0.352 & 0.929 & 0.831 & 0.966 \\
\hline & $\bar{R}$ & 0.002 & 0.004 & 0.003 & 0.003 & 0.003 & 0.002 \\
\hline & $\sigma_{R}$ & 0.019 & 0.023 & 0.019 & 0.020 & 0.020 & 0.019 \\
\hline & Sharpe & 0.115 & 0.169 & 0.169 & 0.160 & 0.175 & 0.113 \\
\hline & $\hat{U}$ & 0.0055 & 0.0068 & 0.0065 & 0.0065 & 0.0067 & 0.0055 \\
\hline \multirow[t]{8}{*}{$\operatorname{HP}(14400)$} & $\hat{\phi}$ & 2.721 & 2.091 & 0.450 & 1.381 & 0.870 & 2.051 \\
\hline & & $(0.587)$ & $(0.621)$ & $(0.384)$ & $(0.293)$ & $(0.625)$ & (1.037) \\
\hline & $\beta_{1}$ & -0.913 & -0.497 & 0.900 & 0.189 & 0.644 & -0.462 \\
\hline & $\beta_{2}$ & 0.408 & 0.868 & 0.435 & 0.982 & 0.765 & 0.887 \\
\hline & $\bar{R}$ & 0.002 & 0.004 & 0.003 & 0.003 & 0.004 & 0.002 \\
\hline & $\sigma_{R}$ & 0.019 & 0.023 & 0.019 & 0.021 & 0.022 & 0.019 \\
\hline & Sharpe & 0.114 & 0.160 & 0.169 & 0.161 & 0.179 & 0.116 \\
\hline & $\hat{U}$ & 0.0055 & 0.0066 & 0.0065 & 0.0065 & 0.0069 & 0.0056 \\
\hline
\end{tabular}


Table 10: Out-of-sample performance of portfolio strategies using recursive Hodrick-Prescott filter

The table presents out-of-sample performance of different portfolio strategies using recursive filtering. For the period April 1963 to June 2011, the Hodrick-Prescott filter are run recursively for every month in the sample and only the last decomposition of these filters is used in $Z_{t}$. Then we divide this sample to two sub-samples. Period April 1963 to June 2001 used for estimating optimal index composition and optimal portfolio rules for each value of index realized at start of each month in this period. Then using this optimal index combination, we construct the value of index for the state variables realized at the start of each month in the period July 2001 to June 2011. Then we implement the same (or similar) portfolio rules estimated for the value of index in the in-sample period (1963-04 to 2001-06), for the realized value of index at the start of each month in the later period (2001-07 to 2011-06) and calculate the corresponding portfolio return over the coming month. This procedure is repeated for all months in the later sample. The returns are stored and used to compute average returns $(\bar{R})$, standard deviations $(\sigma)$, Sharpe ratios (Sharpe), and expected utilities $(\bar{U})$. We use the same state variables and the same filters that we used in the Table 9 .

\begin{tabular}{clrrrrrr}
\hline \hline & & DS & DY & TS & TR & SR & DG \\
\hline Not decomposed & $\bar{R}$ & 0.000 & 0.003 & 0.001 & 0.005 & 0.002 & 0.002 \\
& $\sigma_{R}$ & 0.027 & 0.020 & 0.029 & 0.020 & 0.038 & 0.022 \\
& Sharpe & 0.002 & 0.151 & 0.046 & 0.239 & 0.052 & 0.078 \\
& $\hat{U}$ & -0.0005 & 0.0035 & 0.0008 & 0.0053 & -0.0003 & 0.0021 \\
$\operatorname{HP}(240)$ & $\bar{R}$ & 0.000 & 0.005 & 0.001 & 0.004 & 0.003 & 0.000 \\
& $\sigma_{R}$ & 0.022 & 0.017 & 0.029 & 0.016 & 0.044 & 0.024 \\
& Sharpe & -0.005 & 0.263 & 0.046 & 0.232 & 0.062 & 0.016 \\
& $\hat{U}$ & 0.0002 & 0.0053 & 0.0007 & 0.0047 & -0.0011 & 0.0005 \\
$\operatorname{HP}(900)$ & & & & & & & \\
& $\bar{R}$ & 0.000 & 0.005 & 0.001 & 0.004 & 0.002 & 0.000 \\
& $\sigma_{R}$ & 0.021 & 0.018 & 0.028 & 0.017 & 0.045 & 0.028 \\
& Sharpe & -0.022 & 0.282 & 0.047 & 0.231 & 0.049 & 0.014 \\
& $\hat{U}$ & -0.0001 & 0.0057 & 0.0009 & 0.0048 & -0.0019 & -0.0001 \\
& & & & & & & \\
$\operatorname{HP}(3600)$ & $\bar{R}$ & -0.001 & 0.005 & 0.001 & 0.004 & 0.000 & 0.001 \\
& $\sigma_{R}$ & 0.023 & 0.021 & 0.028 & 0.018 & 0.040 & 0.029 \\
& Sharpe & -0.037 & 0.242 & 0.046 & 0.223 & 0.007 & 0.038 \\
& $\hat{U}$ & -0.0008 & 0.0055 & 0.0008 & 0.0048 & -0.0029 & 0.0005 \\
& & & & & & & \\
& $\bar{R}$ & -0.002 & 0.005 & 0.001 & 0.005 & -0.001 & 0.001 \\
$\operatorname{HP}(14400)$ & $\sigma_{R}$ & 0.028 & 0.020 & 0.030 & 0.020 & 0.041 & 0.027 \\
& Sharpe & -0.061 & 0.263 & 0.048 & 0.239 & -0.018 & 0.044 \\
& $\hat{U}$ & -0.0026 & 0.0059 & 0.0007 & 0.0054 & -0.0041 & 0.0009 \\
\hline
\end{tabular}


Table 11: Optimal index composition using recursive Christiano-Fitzgerald filter for different risk aversion levels

The table is Analogous to Table 6 and presents the GMM estimates of the optimal weights to be used for the long-term and short-term components of a single state variable. The difference is that we use different levels of risk aversion $\gamma=\{1,3,5,10,20\}$, in the CRRA utility function.

\begin{tabular}{|c|c|c|c|c|c|c|c|}
\hline & & $\overline{\mathrm{DS}}$ & $\overline{\text { DY }}$ & $\overline{\text { TS }}$ & $\overline{\text { TR }}$ & $\overline{\text { SR }}$ & $\overline{\mathrm{DG}}$ \\
\hline \multirow[t]{3}{*}{$\gamma=1$} & $\hat{\phi}$ & $\begin{array}{c}1.741 \\
(0.049)\end{array}$ & $\begin{array}{c}1.931 \\
(0.433)\end{array}$ & $\begin{array}{c}0.450 \\
(0.410)\end{array}$ & $\begin{array}{c}1.261 \\
(0.488)\end{array}$ & $\begin{array}{c}0.940 \\
(0.510)\end{array}$ & $\begin{array}{c}2.141 \\
(0.390)\end{array}$ \\
\hline & $\beta_{1}$ & -0.169 & -0.352 & 0.900 & 0.305 & 0.589 & -0.540 \\
\hline & $\beta_{2}$ & 0.986 & 0.936 & 0.435 & 0.952 & 0.808 & 0.842 \\
\hline \multirow[t]{3}{*}{$\gamma=3$} & $\hat{\phi}$ & 0.980 & 1.901 & 0.370 & $\begin{array}{c}1.261 \\
(0432)\end{array}$ & 0.880 & 1.931 \\
\hline & $\beta_{1}$ & 0.557 & -0.324 & 0.932 & 0.305 & 0.637 & -0.352 \\
\hline & $\beta_{2}$ & 0.831 & 0.946 & 0.362 & 0.952 & 0.771 & 0.936 \\
\hline \multirow[t]{3}{*}{$\gamma=5$} & $\hat{\phi}$ & $\begin{array}{c}2.271 \\
(0.340)\end{array}$ & $\begin{array}{c}1.931 \\
(0.391)\end{array}$ & $\begin{array}{c}0.370 \\
(0.329)\end{array}$ & $\begin{array}{c}1.241 \\
(0.357)\end{array}$ & $\begin{array}{c}0.860 \\
(0.427)\end{array}$ & $\begin{array}{c}1.911 \\
(0.461)\end{array}$ \\
\hline & $\beta_{1}$ & -0.644 & -0.352 & 0.932 & 0.324 & 0.652 & -0.334 \\
\hline & $\beta_{2}$ & 0.765 & 0.936 & 0.362 & 0.946 & 0.758 & 0.943 \\
\hline \multirow[t]{3}{*}{$\gamma=10$} & $\hat{\phi}$ & $\begin{array}{c}2.341 \\
(0.380)\end{array}$ & $\begin{array}{c}1.981 \\
(0.380)\end{array}$ & $\begin{array}{c}0.320 \\
(0.442)\end{array}$ & $\begin{array}{c}1.221 \\
(0.476)\end{array}$ & $\begin{array}{c}0.840 \\
(0.357)\end{array}$ & $\begin{array}{c}1.901 \\
(0.504)\end{array}$ \\
\hline & $\beta_{1}$ & -0.696 & -0.399 & 0.949 & 0.343 & 0.667 & -0.324 \\
\hline & $\beta_{2}$ & 0.718 & 0.917 & 0.315 & 0.939 & 0.745 & 0.946 \\
\hline \multirow[t]{3}{*}{$\gamma=20$} & $\hat{\phi}$ & $\begin{array}{c}2.341 \\
(0.339)\end{array}$ & $\begin{array}{c}2.151 \\
(0.342)\end{array}$ & $\begin{array}{c}0.320 \\
(0.314)\end{array}$ & $\begin{array}{c}1.201 \\
(0.483)\end{array}$ & $\begin{array}{c}0.700 \\
(0.513)\end{array}$ & $\begin{array}{c}1.851 \\
(0.634)\end{array}$ \\
\hline & $\beta_{1}$ & -0.696 & -0.548 & 0.949 & 0.362 & 0.765 & -0.276 \\
\hline & $\beta_{2}$ & 0.718 & 0.836 & 0.315 & 0.932 & 0.644 & 0.961 \\
\hline
\end{tabular}


Table 12: In-sample performance of portfolio strategies using recursive Christiano-Fitzgerald for different risk aversion levels

The table is Analogous to Table 7 and presents the in-sample performance of portfolio strategies based on different filter and state variable pairs. The difference here is that we use different utility specifications as investors objective. We use a CRRA utility function with investors relative risk aversion, $\gamma$, gets a range of values from $\{1,3,5,10,20\}$. The weights used for $\beta^{\prime} Z_{t}$ are those of the recursive decomposition from Table 11.

\begin{tabular}{clrrrrrr}
\hline \hline & & DS & DY & TS & TR & SR & DG \\
\hline Not decomposed & $\bar{R}$ & 0.002 & 0.002 & 0.003 & 0.004 & 0.003 & 0.002 \\
$\gamma=5$ & $\sigma_{R}$ & 0.019 & 0.018 & 0.019 & 0.023 & 0.019 & 0.017 \\
& Sharp & 0.108 & 0.125 & 0.169 & 0.157 & 0.140 & 0.126 \\
& $\hat{U}$ & 0.0054 & 0.0057 & 0.0065 & 0.0064 & 0.0060 & 0.0057 \\
$\gamma=1$ & & & & & & & \\
& $\bar{R}$ & 0.004 & 0.005 & 0.005 & 0.004 & 0.005 & 0.005 \\
& $\sigma_{R}$ & 0.043 & 0.034 & 0.042 & 0.033 & 0.041 & 0.042 \\
& Sharp & 0.087 & 0.137 & 0.115 & 0.134 & 0.111 & 0.113 \\
& $\hat{U}$ & 0.0071 & 0.0084 & 0.0082 & 0.0082 & 0.0080 & 0.0082 \\
$\gamma=3$ & & & & & & & \\
& $\bar{R}$ & 0.002 & 0.004 & 0.004 & 0.004 & 0.004 & 0.004 \\
& $\sigma_{R}$ & 0.026 & 0.023 & 0.024 & 0.024 & 0.028 & 0.027 \\
& Sharp & 0.095 & 0.179 & 0.155 & 0.173 & 0.145 & 0.131 \\
& $\hat{U}$ & 0.0057 & 0.0076 & 0.0071 & 0.0076 & 0.0071 & 0.0067 \\
& & & & & & & \\
$\gamma=5$ & $\bar{R}$ & 0.003 & 0.004 & 0.003 & 0.004 & 0.003 & 0.003 \\
& $\sigma_{R}$ & 0.020 & 0.018 & 0.019 & 0.018 & 0.021 & 0.020 \\
& Sharp & 0.129 & 0.206 & 0.168 & 0.193 & 0.157 & 0.145 \\
& $\hat{U}$ & 0.0058 & 0.0071 & 0.0065 & 0.0070 & 0.0064 & 0.0061 \\
& & & & & & & \\
$\gamma=10$ & $\bar{R}$ & 0.002 & 0.003 & 0.002 & 0.002 & 0.002 & 0.002 \\
& $\sigma_{R}$ & 0.011 & 0.012 & 0.014 & 0.012 & 0.014 & 0.012 \\
& Sharp & 0.135 & 0.213 & 0.176 & 0.200 & 0.163 & 0.162 \\
& $\hat{U}$ & 0.0051 & 0.0060 & 0.0057 & 0.0059 & 0.0055 & 0.0054 \\
& & & & & & & \\
& $\bar{R}$ & 0.001 & 0.001 & 0.001 & 0.001 & 0.001 & 0.001 \\
& $\sigma_{R}$ & 0.006 & 0.007 & 0.007 & 0.007 & 0.007 & 0.006 \\
& Sharp & 0.134 & 0.204 & 0.186 & 0.196 & 0.166 & 0.170 \\
& $\hat{U}$ & 0.0045 & 0.0051 & 0.0049 & 0.0050 & 0.0047 & 0.0047 \\
\hline
\end{tabular}


Table 13: Optimal index composition using recursive Christiano-Fitzgerald filter for 3-month investment horizon

The table is Analogous to Table 6 and presents the GMM estimates of the optimal weights to be used for the long-term and short-term components of a single state variable. The difference here is that we use a 3-month buy-and-hold investment horizon, so we use 3-month non-overlapping returns for stock and bond returns. In total we have 193 non-overlapping periods for estimating the optimal index composition and corresponding standard errors.

\begin{tabular}{|c|c|c|c|c|c|c|c|}
\hline & & DS & DY & TS & TR & SR & DG \\
\hline \multirow[t]{3}{*}{$\mathrm{CF}(6)$} & $\hat{\phi}$ & $\begin{array}{c}1.231 \\
(0.919)\end{array}$ & $\begin{array}{c}2.331 \\
(0.648)\end{array}$ & $\begin{array}{c}0.470 \\
(0.460)\end{array}$ & $\begin{array}{c}1.351 \\
(0.398)\end{array}$ & $\begin{array}{c}0.940 \\
(0.626)\end{array}$ & $\begin{array}{c}1.221 \\
(0.440)\end{array}$ \\
\hline & $\beta_{1}$ & 0.334 & -0.689 & 0.891 & 0.218 & 0.589 & 0.343 \\
\hline & $\beta_{2}$ & 0.943 & 0.725 & 0.453 & 0.976 & 0.808 & 0.939 \\
\hline \multirow[t]{3}{*}{$\mathrm{CF}(12)$} & $\hat{\phi}$ & $\begin{array}{c}2.901 \\
(0.607)\end{array}$ & $\begin{array}{c}2.681 \\
(0.537)\end{array}$ & $\begin{array}{c}0.250 \\
(0.404)\end{array}$ & $\begin{array}{c}1.101 \\
(0.684)\end{array}$ & $\begin{array}{c}0.680 \\
(0.682)\end{array}$ & $\begin{array}{c}2.391 \\
(1.029)\end{array}$ \\
\hline & $\beta_{1}$ & -0.971 & -0.896 & 0.969 & 0.453 & 0.777 & -0.731 \\
\hline & $\beta_{2}$ & 0.238 & 0.444 & 0.248 & 0.891 & 0.629 & 0.682 \\
\hline \multirow[t]{3}{*}{$\mathrm{CF}(24)$} & $\hat{\phi}$ & $\begin{array}{c}2.111 \\
(1.786)\end{array}$ & $\begin{array}{c}2.331 \\
(0.742)\end{array}$ & $\begin{array}{c}0.040 \\
(0.425)\end{array}$ & $\begin{array}{c}1.141 \\
(0.715)\end{array}$ & $\begin{array}{c}0.840 \\
(0.561)\end{array}$ & $\begin{array}{c}1.551 \\
(0.560)\end{array}$ \\
\hline & $\beta_{1}$ & -0.514 & -0.689 & 0.999 & 0.417 & 0.667 & 0.020 \\
\hline & $\beta_{2}$ & 0.858 & 0.725 & 0.040 & 0.909 & 0.745 & 1.000 \\
\hline \multirow[t]{3}{*}{$\mathrm{CF}(36)$} & $\hat{\phi}$ & $\begin{array}{c}2.181 \\
(0.664)\end{array}$ & $\begin{array}{c}2.951 \\
(0.724)\end{array}$ & $\begin{array}{c}0.160 \\
(0.448)\end{array}$ & $\begin{array}{c}1.251 \\
(0.881)\end{array}$ & $\begin{array}{c}0.920 \\
(0.439)\end{array}$ & $\begin{array}{c}2.631 \\
(0.770)\end{array}$ \\
\hline & $\beta_{1}$ & -0.573 & -0.982 & 0.987 & 0.315 & 0.605 & -0.873 \\
\hline & $\beta_{2}$ & 0.819 & 0.189 & 0.159 & 0.949 & 0.796 & 0.488 \\
\hline \multirow[t]{3}{*}{$\mathrm{CF}(48)$} & $\hat{\phi}$ & $\begin{array}{c}0.220 \\
(0.802)\end{array}$ & $\begin{array}{c}2.601 \\
(0.656)\end{array}$ & $\begin{array}{c}0.370 \\
(0.439)\end{array}$ & $\begin{array}{c}1.841 \\
(0.788)\end{array}$ & $\begin{array}{c}0.890 \\
(0.379)\end{array}$ & $\begin{array}{c}1.061 \\
(1.407)\end{array}$ \\
\hline & $\beta_{1}$ & 0.976 & -0.858 & 0.932 & -0.267 & 0.629 & 0.488 \\
\hline & $\beta_{2}$ & 0.218 & 0.514 & 0.362 & 0.964 & 0.777 & 0.873 \\
\hline \multirow[t]{3}{*}{$\mathrm{CF}(72)$} & $\hat{\phi}$ & $\begin{array}{c}0.150 \\
(0.775)\end{array}$ & $\begin{array}{c}3.002 \\
(0.641)\end{array}$ & $\begin{array}{c}0.530 \\
(0.386)\end{array}$ & $\begin{array}{c}2.461 \\
(0.262)\end{array}$ & $\begin{array}{c}1.011 \\
(0.477)\end{array}$ & $\begin{array}{c}2.191 \\
(0.633)\end{array}$ \\
\hline & $\beta_{1}$ & 0.989 & -0.990 & 0.863 & -0.777 & 0.531 & -0.581 \\
\hline & $\beta_{2}$ & 0.150 & 0.140 & 0.506 & 0.629 & 0.847 & 0.814 \\
\hline
\end{tabular}


Table 14: In-sample performance of portfolio choice strategies using recursive ChristianoFitzgerald filter for 3-month investment horizon

The table is Analogous to Table 7 and presents the in-sample performance of portfolio strategies based on different filter and state variable pairs. The difference here is that we use a 3-month buy-and-hold investment horizon, so we use 3-month non-overlapping returns for stock and bond returns. In total we have 193 nonoverlapping periods for estimating the optimal index composition and corresponding standard errors. The weights used for $\beta^{\prime} Z_{t}$ are those of the recursive decomposition from Table 13.

\begin{tabular}{|c|c|c|c|c|c|c|c|}
\hline & & $\overline{D S}$ & DY & TS & TR & SR & DG \\
\hline \multirow[t]{4}{*}{ Not decomposed } & $\bar{R}$ & 0.002 & 0.003 & 0.006 & 0.003 & 0.003 & $\overline{0.002}$ \\
\hline & $\sigma_{R}$ & 0.019 & 0.018 & 0.026 & 0.020 & 0.020 & 0.013 \\
\hline & Sharpe & 0.096 & 0.168 & 0.212 & 0.144 & 0.156 & 0.114 \\
\hline & $\hat{U}$ & 0.0178 & 0.0189 & 0.0205 & 0.0185 & 0.0188 & 0.0178 \\
\hline \multirow{4}{*}{$\mathrm{CF}(6)$} & $\bar{R}$ & 0.002 & 0.004 & 0.006 & 0.004 & 0.005 & 0.003 \\
\hline & $\sigma_{R}$ & 0.019 & 0.019 & 0.024 & 0.017 & 0.024 & 0.018 \\
\hline & Sharpe & 0.078 & 0.214 & 0.227 & 0.204 & 0.202 & 0.154 \\
\hline & $\hat{U}$ & 0.0173 & 0.0198 & 0.0206 & 0.0195 & 0.0199 & 0.0186 \\
\hline \multirow[t]{4}{*}{$\mathrm{CF}(12)$} & $\bar{R}$ & 0.002 & 0.004 & 0.006 & 0.004 & 0.004 & 0.002 \\
\hline & $\sigma_{R}$ & 0.014 & 0.019 & 0.026 & 0.018 & 0.023 & 0.018 \\
\hline & Sharpe & 0.123 & 0.210 & 0.214 & 0.224 & 0.158 & 0.117 \\
\hline & $\hat{U}$ & 0.0180 & 0.0198 & 0.0206 & 0.0200 & 0.0189 & 0.0180 \\
\hline \multirow[t]{4}{*}{$\mathrm{CF}(24)$} & $\bar{R}$ & 0.002 & 0.004 & 0.006 & 0.003 & 0.004 & 0.002 \\
\hline & $\sigma_{R}$ & 0.014 & 0.021 & 0.025 & 0.017 & 0.023 & 0.018 \\
\hline & Sharpe & 0.172 & 0.189 & 0.223 & 0.187 & 0.176 & 0.117 \\
\hline & $\hat{U}$ & 0.0187 & 0.0196 & 0.0207 & 0.0192 & 0.0192 & 0.0181 \\
\hline \multirow[t]{4}{*}{$\mathrm{CF}(36)$} & $\bar{R}$ & 0.002 & 0.003 & 0.005 & 0.003 & 0.005 & 0.001 \\
\hline & $\sigma_{R}$ & 0.014 & 0.019 & 0.025 & 0.016 & 0.025 & 0.012 \\
\hline & Sharpe & 0.122 & 0.142 & 0.216 & 0.172 & 0.206 & 0.093 \\
\hline & $\hat{U}$ & 0.0180 & 0.0186 & 0.0205 & 0.0189 & 0.0200 & 0.0175 \\
\hline \multirow[t]{4}{*}{$\mathrm{CF}(48)$} & $\bar{R}$ & 0.002 & 0.003 & 0.006 & 0.003 & 0.006 & 0.001 \\
\hline & $\sigma_{R}$ & 0.018 & 0.021 & 0.027 & 0.022 & 0.027 & 0.011 \\
\hline & Sharpe & 0.098 & 0.127 & 0.211 & 0.158 & 0.215 & 0.122 \\
\hline & $\hat{U}$ & 0.0178 & 0.0182 & 0.0206 & 0.0191 & 0.0203 & 0.0178 \\
\hline \multirow[t]{4}{*}{$\mathrm{CF}(72)$} & $\bar{R}$ & 0.002 & 0.003 & 0.006 & 0.003 & 0.006 & 0.002 \\
\hline & $\sigma_{R}$ & 0.017 & 0.020 & 0.027 & 0.015 & 0.026 & 0.016 \\
\hline & Sharpe & 0.099 & 0.145 & 0.212 & 0.185 & 0.230 & 0.133 \\
\hline & $\hat{U}$ & 0.0178 & 0.0186 & 0.0206 & 0.0190 & 0.0207 & 0.0182 \\
\hline
\end{tabular}




\section{A Appendix: Further Results and Numerical Implemen- tation}

\section{A.1 Using filters over the whole sample}

In Section 5, we use a recursive filter to decompose state variables into their long-term and short-term components. This enables us to actually use the decomposition for investment purposes. But it also has some disadvantages. In recursive filtering, we use data up to the start of each month to determine the long and and short-term components. This makes the decomposition particularly vulnerable to the end-point sensitivity of the Christiano-Fitzgerald and Hodrick-Prescott filter. This makes recursive filtering less smooth compared to running the filter once over the whole sample. To assess how our results are affected by the method of filtering, we perform our analysis using filters run once over the the whole sample. This method is infeasible and cannot be performed in a practical investment context. We already presented the filtering results over the whole sample in Section 3.1.

We first present the results for the Christiano-Fitzgerald filter. As we did for the recursive filtering procedure, we perform a simple regression of returns on each of the state variables, as well as on the long-term and short-term components of each state variable. This is done in Table A1 for stock returns using the familiar range of Christiano-Fitzgerald filters. The results are surprisingly good for some state variables such as the log dividend yield (DY) and the stock market trend (TR). We observe huge improvement in $R^{2} s$ when we regress stock returns on the two components compare to the $R^{2} s$ in top line of the table, where we regress stock returns on the state variable itself. A similar result is presented in Table A2 for bond returns. Here we also see a substantial improvement in the $R^{2}$ s for some state variables like the term spread and short rate. The explanation for substantial extra predictability is that the Christiano-Fitzgerald filter (as well as the Hodrick-Prescott filter) is effectively a smoother rather than a filter and therefore uses both past and future information of the state variables. The smoothed state variables therefore include future price levels via DY and TR, which work best in Table A1 for stock returns, and future interest rate levels via SR and TS, which work best in Table Table A2 for bond returns.

Using the new values of the short-term and long-term components, we estimate the optimal index using various versions of the CF filter. The results are presented in Figure A1 and Table A3. As expected, the results are much stronger compare to the case of using recursive filtering. The loading of the short-term component $\left(\beta_{2}\right)$ is much larger in absolute size than that of the long-term component $\left(\beta_{1}\right)$ for all state variables considered with the exception of 
the term spread. Also, for a number of state variables (DS, TR, SR, DG) the loadings of the long-term and short-term components are of opposite signs.

If we consider the patterns for the log dividend yield (DY) in Figure A1, the value of $\phi$ is very stable across different filters and also the standard error band is robust. For all filters, we cannot reject the hypothesis that it is only the short-term component that matters for asset allocation, but we can reject that only the long-term component matters or that both components are equally important (whether weighted or unweighted). The result for the short rate (SR) is completely analogous to that for the log dividend yield (DY). For the stock market trend (TR), except for the $\mathrm{CF}(72)$ filter, we can reject that only the long-term component matters, but also that it is only the short-term component. Again, we also reject the hypothesis that both the long-term and short-term component are equally important, whether weighted or unweighted. In particular, the sign of the two components in the optimal index $\beta^{\prime} Z_{t}$ is significantly different. The results for dividend growth (DG) very much resemble those for the trend (TR). The patterns are more jagged for the default spread (DS) and the term spread (TS).

Using the weight vectors $\beta$, the optimal fractions invested in each of our three asset categories as a function of the index $\beta^{\prime} Z_{t}$ for $\mathrm{CF}(12)$ are shown in Figure A2. The in-sample performance of different portfolio strategies are presented in Table A4. We see that particularly for the dividend yield (DY), the stock market trend (TR), and to some extent for the short rate (SR) the results improve substantially. The magnitude of the improvement varies with the filter that is used. Given the short horizon of the investor in this example (one month), the best results appear to be achieved by the $\mathrm{CF}(x)$ filters that use smaller value of $x$. The Sharpe ratios for DY and TR increase by more than $100 \%$ for decompositions with a short-term up to one-year. The same holds for the expected utilities. If the short-term component is defined more loosely to periods up to 3 or even 7 years, the advantage of using the long-term short-term decompositions disappears for investors with a one-month horizon. Particularly for the $\mathrm{CF}(72)$ filter, the results are roughly similar to the setting where the state variables are not decomposed at all.

Finally we provide the same results using the Hodrick-Prescott filter. Estimates of the optimal index coefficient and also the in-sample performance are provided in Table A5. The results are similar to those obtained by using the Christiano-Fitzgerald filter. The main difference is that for the term spread, the loading of the long-term component is lower than or equal to the loading of the short-term component. The effect of the smoothing parameter is not uniform across different state variables. For some state variables (DY, TR, SR), less smoothing results 
in better Sharpe ratios. For other state variables, more (TS) smoothing is better or smoothing does not matter at all (DS, DG).

\section{A.2 Numerical implementation}

For the weights $\omega_{t}(z)=\omega\left(\beta^{\prime} Z_{t}, z\right)$ used in (4) and (5), we take a Gaussian kernel

$$
\omega(y, z)=(2 \pi)^{-1 / 2} \exp \left(\frac{-u^{2}}{2}\right) .
$$

where $u=(y-z) / h$ and $h$ is the so-called bandwidth. The choice of bandwidth has a direct effect on the optimal value of $\beta$. A high value of $h$ leads to smoother weights in the conditional moment condition, and therefore to smaller differences between states. A low value of $h$, on the other hand, leads to more differentiation between states, but also uses less observations in determining moment conditions. Brandt (1999) proposes the bandwidth to be chosen as

$$
h=\lambda_{b} \sigma_{z} n^{(1 /(K+4))}
$$

where $\sigma_{z}$ is the standard deviation of $z, n$ is the number of observations, $K$ is the dimension of vector of state variables (that is one in our context), and the $\lambda_{b}$ is the parameter that should be chosen to minimize the standard deviation of $\beta$. We have experimented with different values of $\lambda_{b}=1,3,5$ that lead to different values of the bandwidth parameter $h$. Some illustrative examples of the resulting objective functions for different values of the bandwidth parameter are provided in Figure A3 for term spread (TS) and CF(12) and CF(72).

For $\lambda_{b}=5$, the objective function is relatively smooth. The objective function is more jagged for $\lambda_{b}=1$ with a number of local maxima. Therefore we used $\lambda_{b}=3$ as a benchmark in our empirical analysis, which strikes a balance between these two. Fortunately, most of the time, the choice of $\lambda_{b}$ does not change the optimal $\phi$ substantially, and therefore has a minor effect on the relative importance of short-term and long-term components for asset allocation decisions.

We use double scaling. First, the elements of $Z_{t}$ are divided by their time-series standard deviation to make the different components comparable. Next, for a given value of $\beta$, the index $\beta^{\prime} Z_{t}$ is standardized by its time-series standard deviation before the weights are computed according to (A1).

To make the computation time of the GMM estimation procedure feasible, we use interpolation. For a given value of $\beta$, we compute the weights for the weighted constrained optimization problem (10). Next, we put a grid of values for $z$ ranging from the lowest to the highest value of $\beta^{\prime} Z_{t}$ obtained in the sample. For each of the grid points, we compute the optimal constrained 
asset allocation using a numerical optimizer for quadratic programming. We used the programming package Ox, see Doornik (2007) and Doornik and Ooms (2007). The optimal solutions for values of $z$ between the grid points are obtained by linear interpolation.

Once all the constrained and possibly interpolated optimal asset allocations are obtained, we numerically solve for the corresponding Lagrange-Kuhn-Tucker multipliers. This is easily done by solving a linear system of equations.

The derivatives of the objective function needed to compute the standard errors are obtained by numerical differentiation. The GMM criterion function for $\beta$ can become very small numerically, resulting in instabilities particularly for the initial choices of the weighting matrix. We avoid this problem by re-scaling the objective function to be numerically more stable. The problem generally disappears after the second round estimation of the weighting matrix, because the different scales of the elements of the moment vectors are then accounted for by the weighting matrix, which captures the variance of each element of the moment vector. 


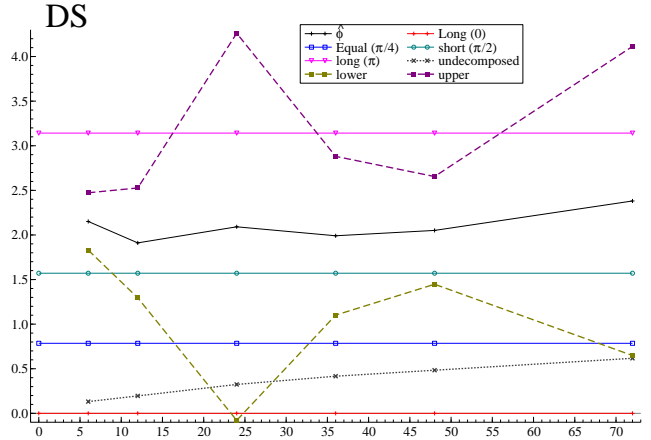

TS
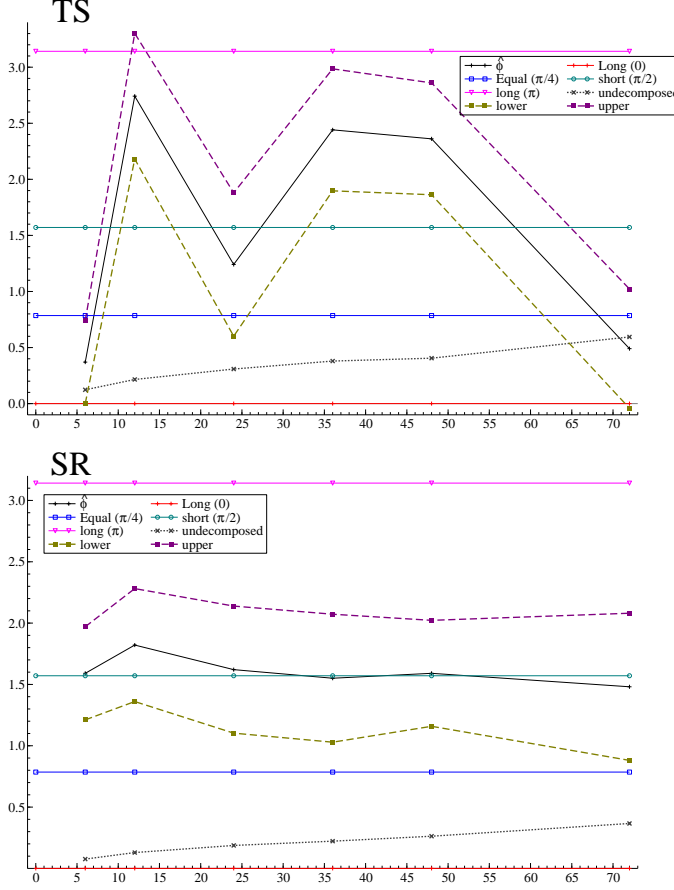

DY

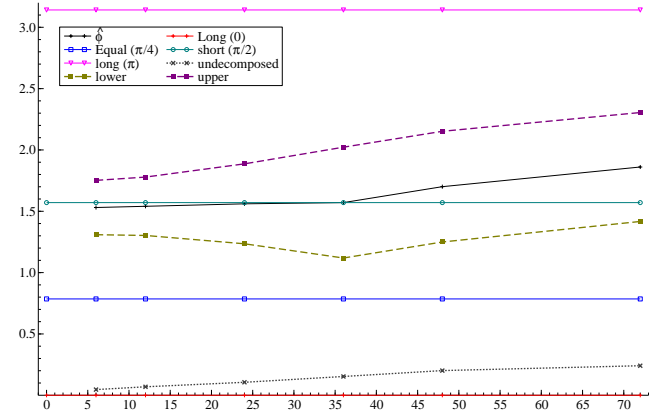

TR
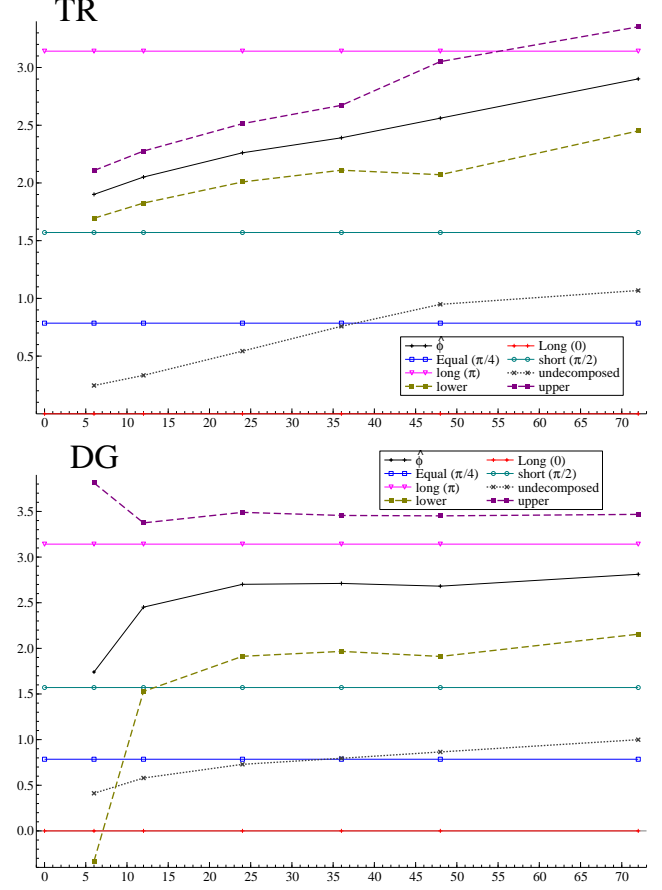

Figure A1: Estimated angle for short-term and long-term components using ChristianoFitzgerald filter over the whole sample

This graph is similar to Figure 8 and shows the the optimal angle $\phi$ for the long-term and short-term component weights $\beta_{1}=\cos (\phi)$ and $\beta_{2}=\sin (\phi)$, respectively, and two times its standard error band (dashed), for different state variables and filters. The horizontal axis gives the value of $x$, where $\mathrm{CF}(x)$ is the Christiano-Fitzgerald filter used for the GMM estimation of $\phi$. The difference here is that Christiano-Fitzgerald filter run once over the entire sample to estimate the long and short-term components. The panels are for the different state variables: default spread (DS, upper-left), log dividend-to-price ratio (DY, upper-right), term spread (TS, midleft), stock market trend (TR, mid-right), short rate (SR, lower-left), dividend growth rate (DG, lower-right). The horizontal lines correspond to the different null hypotheses of interest: only the long-term component matters $(\phi=0$ or $\phi=\pi)$, only the short-term component matters $(\phi=\pi / 2)$, both (scaled) components matter equally $(\phi=\pi / 4)$, both (unscaled) components matter equally, such that the original state variable suffices $\left(\tan (\phi)=\sigma_{L} / \sigma_{H}\right)$. 

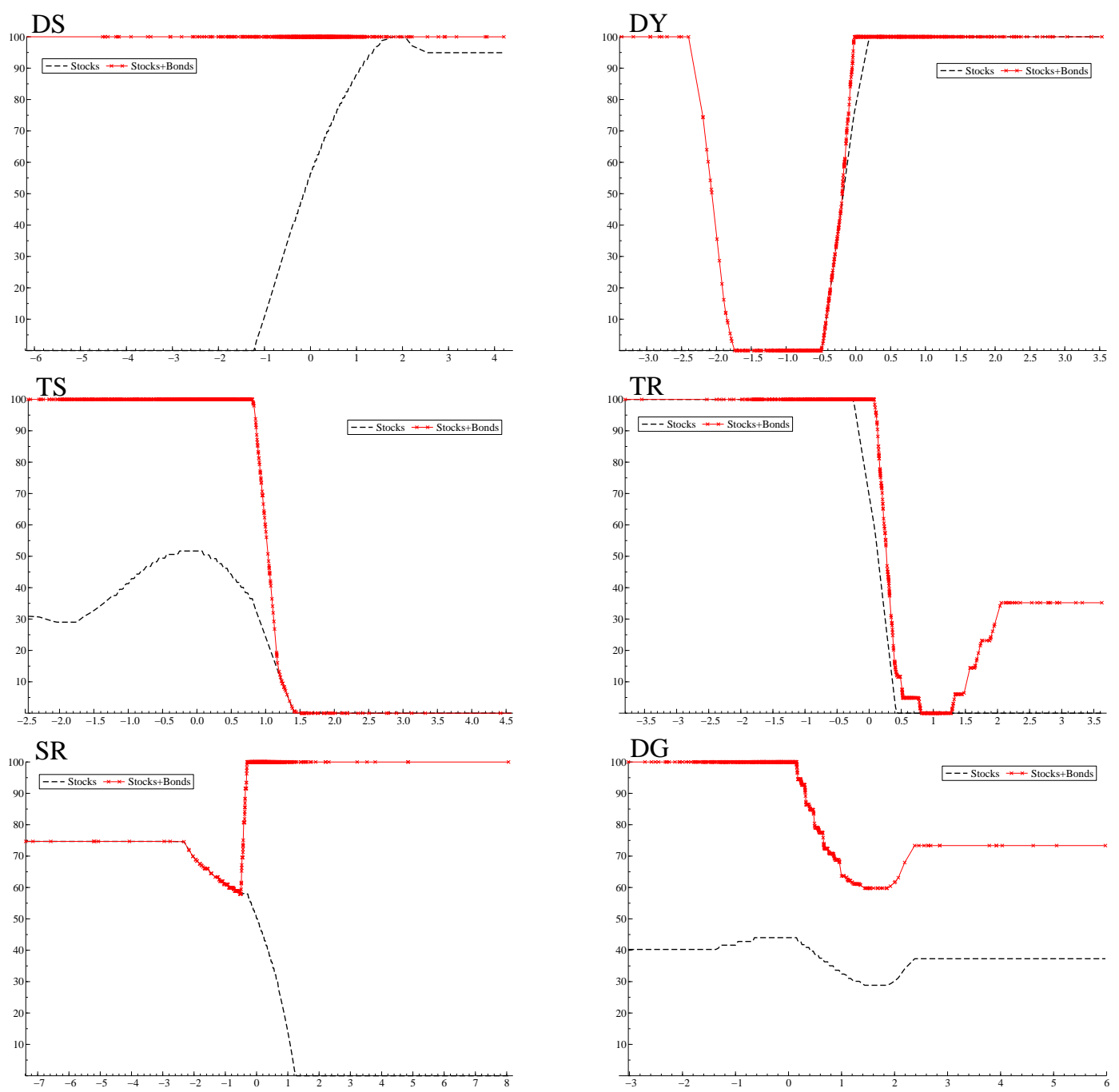

Figure A2: Optimal investment strategies for filter $\mathrm{CF}(12)$ using Christiano-Fitzgerald filter over the whole sample

Each graph presents the optimal asset allocation as a function of the index $\beta^{\prime} Z_{t}$ that is computed using the optimal $\beta$ from Table A3 and $Z_{t}$ holding the long-term and short-term components of the state variable. The $\mathrm{CF}(12)$ filter is used for the decomposition. The difference here is that Christiano-Fitzgerald filter run once over the entire sample to estimate the long and short-term components. The index $\beta^{\prime} Z_{t}$ is standardized by dividing by its time-series standard deviation. The horizontal axis gives the value of standardized $\beta^{\prime} Z_{t}$. The vertical axis gives the percentage invested in stocks and stocks plus bonds. The panels are for the different state variables: default spread (DS, upper-left), log dividend-to-price ratio (DY, upper-right), term spread (TS, mid-left), stock market trend (TR, mid-right), short rate (SR, lower-left), dividend growth rate (DG, lower-right). 

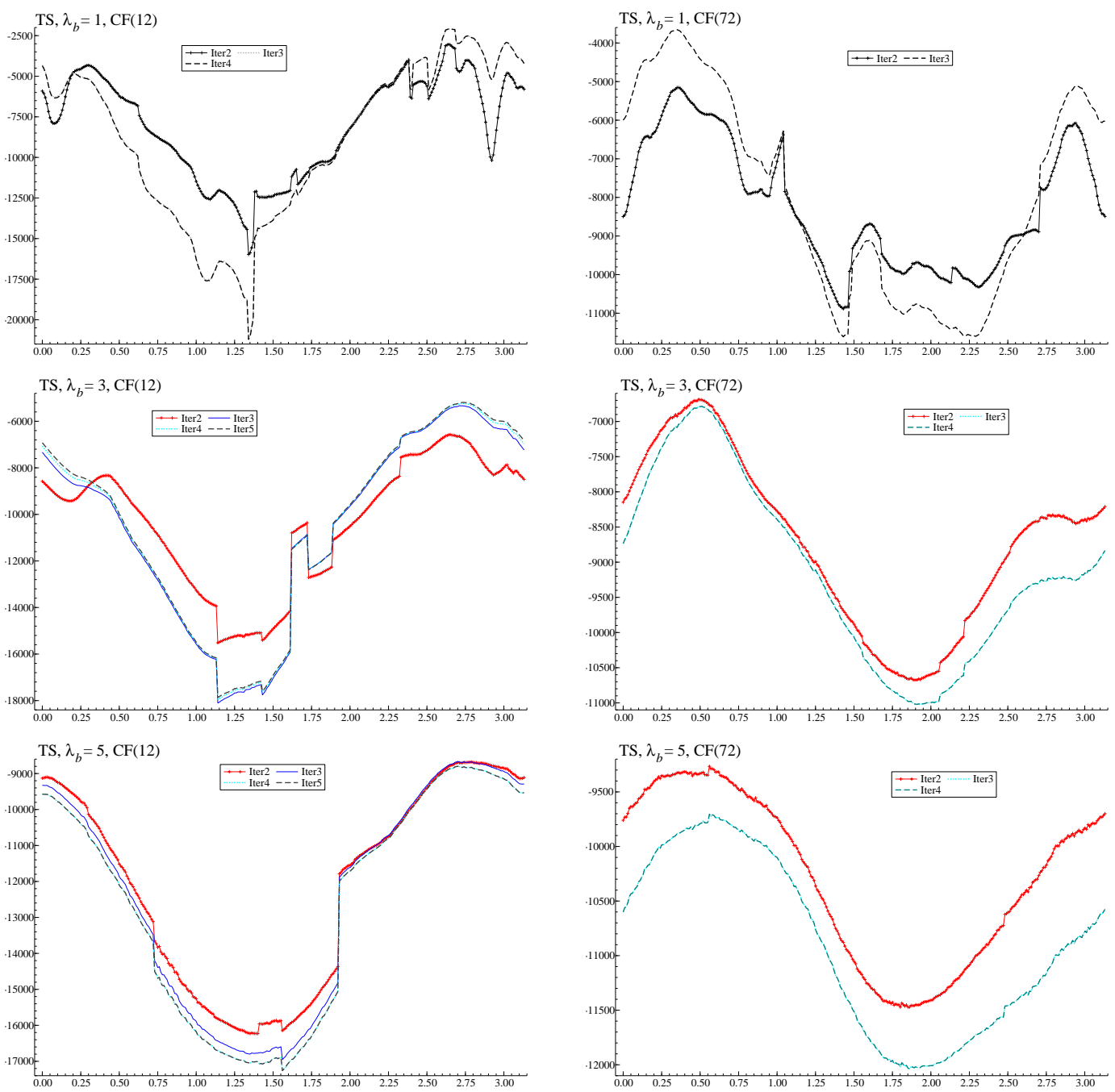

Figure A3: GMM objective functions for term spread and the different bandwidths

This figure presents plots of the GMM objective function as a function of the angle $\phi$ for term spread (TS) and different $\lambda_{b}$ that determine the bandwidth parameter as described in Equation (A2). The results are presented for filters $\mathrm{CF}$ (12) (left) and $\mathrm{CF}$ (72) (right). For each filter, the upper, middle and lower graphs correspond to $\lambda_{b}=1,3$, and 5 respectively. 
Table A1: Regressions of stock return on long-term and short-term components using Christiano-Fitzgerald filter over the whole sample

This table reports the $R^{2}$ and the partial $R^{2}$ values $R_{L, p}^{2}$ and $R_{S, p}^{2}$ of the long-term and short-term component of state variables, respectively. The partial $R^{2}$ measures the contribution of a variable after all other variables have already been included in the model. The state variables are the default spread (DS), log dividend yield (DY), term spread (TS), stock market trend (TR), short rate (SR), and dividend growth (DG). To decompose each state variable into its long-term and short-term component, we use the Christiano-Fitzgerald filter (once over the whole sample), where the short-term is defined as up to $x$ months for $x=6,12,24,36,48,72$. The top block of entries gives the results for the non-decomposed state variables. The sample covers April 1953 to June 2011.

\begin{tabular}{clrrrrrr}
\hline \hline Not & & DS & DY & TS & TR & SR & DG \\
\hline decomposed & & 0.000 & 0.005 & 0.013 & 0.002 & 0.010 & 0.000 \\
CF(6) & & & & & & & \\
& $R_{L, p}^{2}$ & 0.000 & 0.003 & 0.011 & 0.051 & 0.008 & 0.000 \\
& $R_{S, p}^{2}$ & 0.023 & 0.280 & 0.013 & 0.336 & 0.031 & 0.001 \\
& $R^{2}$ & 0.023 & 0.281 & 0.023 & 0.359 & 0.039 & 0.001 \\
$\mathrm{CF}(12)$ & & & & & & & \\
& $R_{L, p}^{2}$ & 0.000 & 0.001 & 0.010 & 0.058 & 0.007 & 0.000 \\
& $R_{S, p}^{2}$ & 0.021 & 0.260 & 0.009 & 0.244 & 0.025 & 0.000 \\
& $R^{2}$ & 0.022 & 0.261 & 0.018 & 0.278 & 0.031 & 0.000 \\
$\mathrm{CF}(24)$ & & & & & & & \\
& $R_{L, p}^{2}$ & 0.001 & 0.001 & 0.006 & 0.070 & 0.005 & 0.000 \\
& $R_{S, p}^{2}$ & 0.021 & 0.171 & 0.019 & 0.114 & 0.034 & 0.000 \\
& $R^{2}$ & 0.022 & 0.171 & 0.024 & 0.167 & 0.039 & 0.000 \\
$\mathrm{CF}(36)$ & & & & & & & \\
& $R_{L, p}^{2}$ & 0.002 & 0.000 & 0.003 & 0.051 & 0.003 & 0.003 \\
& $R_{S, p}^{2}$ & 0.018 & 0.097 & 0.028 & 0.032 & 0.048 & 0.003 \\
& $R^{2}$ & 0.020 & 0.098 & 0.031 & 0.078 & 0.051 & 0.007 \\
& & & & & & & \\
$\mathrm{CF}(48)$ & $R_{L, p}^{2}$ & 0.003 & 0.000 & 0.003 & 0.036 & 0.002 & 0.007 \\
& $R_{S, p}^{2}$ & 0.017 & 0.056 & 0.031 & 0.008 & 0.051 & 0.006 \\
& $R^{2}$ & 0.019 & 0.056 & 0.034 & 0.042 & 0.053 & 0.012 \\
& & & & & & & \\
$\mathrm{CF}(72)$ & $R_{L, p}^{2}$ & 0.006 & 0.000 & 0.001 & 0.023 & 0.001 & 0.011 \\
& $R_{S, p}^{2}$ & 0.018 & 0.037 & 0.028 & 0.002 & 0.037 & 0.005 \\
& $R^{2}$ & 0.023 & 0.038 & 0.029 & 0.024 & 0.038 & 0.015 \\
\hline
\end{tabular}


Table A2: Regressions of bond returns on long-term and short-term components using Christiano-Fitzgerald filter over the whole sample

This table reports the $R^{2}$ and the partial $R^{2}$ values resulting from regression of the bond excess return on each state variables as well as on the long-term and short-term component of each state variable. The partial $R^{2}$ (denoted by $R_{L, p}^{2}$ and $R_{S, p}^{2}$ for the long-term and short-term component respectively) measures the contribution of a variable after all other variables have already been included in the model. The state variables are the default spread (DS), log dividend yield (DY), term spread (TS), stock market trend (TR), short rate (SR), and dividend growth (DG). To decompose each state variable into its long-term and short-term component, we use the Christiano-Fitzgerald filter (once over the whole sample), where the short-term is defined as up to $x$ months for $x=6,12,24,36,48,72$. The top block of entries gives the results for the non-decomposed state variables. The sample covers April 1953 to June 2011.

\begin{tabular}{ccrrrrrr}
\hline \hline & & DS & DY & TS & TR & SR & DG \\
\hline decomposed & $R^{2}$ & 0.007 & 0.000 & 0.013 & 0.011 & 0.000 & 0.000 \\
& & & & & & & \\
CF(6) & $R_{L, p}^{2}$ & 0.010 & 0.001 & 0.017 & 0.007 & 0.002 & 0.000 \\
& $R_{S, p}^{2,}$ & 0.015 & 0.003 & 0.016 & 0.010 & 0.098 & 0.001 \\
& $R^{2}$ & 0.024 & 0.003 & 0.032 & 0.017 & 0.099 & 0.001 \\
$\mathrm{CF}(12)$ & $R_{L, p}^{2}$ & 0.008 & 0.001 & 0.022 & 0.005 & 0.003 & 0.001 \\
& $R_{S, p}^{2}$ & 0.001 & 0.005 & 0.023 & 0.015 & 0.095 & 0.001 \\
& $R^{2}$ & 0.008 & 0.006 & 0.044 & 0.019 & 0.097 & 0.002 \\
$\mathrm{CF}(24)$ & & & & & & & \\
& $R_{L, p}^{2}$ & 0.006 & 0.001 & 0.025 & 0.001 & 0.003 & 0.000 \\
& $R_{S, p}^{2}$ & 0.001 & 0.012 & 0.016 & 0.022 & 0.053 & 0.000 \\
& $R^{2}$ & 0.007 & 0.013 & 0.040 & 0.023 & 0.056 & 0.000 \\
& & & & & & & \\
$\mathrm{CF}(36)$ & $R_{L, p}^{2}$ & 0.004 & 0.004 & 0.027 & 0.000 & 0.004 & 0.000 \\
& $R_{S, p}^{2}$ & 0.003 & 0.024 & 0.011 & 0.026 & 0.040 & 0.000 \\
& $R^{2}$ & 0.007 & 0.026 & 0.037 & 0.026 & 0.043 & 0.000 \\
& & & & & & & \\
$\mathrm{CF}(48)$ & $R_{L, p}^{2}$ & 0.004 & 0.003 & 0.028 & 0.000 & 0.004 & 0.000 \\
& $R_{S, p}^{2}$ & 0.003 & 0.023 & 0.011 & 0.019 & 0.029 & 0.000 \\
& $R^{2}$ & 0.007 & 0.026 & 0.038 & 0.019 & 0.032 & 0.000 \\
& & & & & & & \\
$\mathrm{CF}(72)$ & $R_{L, p}^{2}$ & 0.002 & 0.003 & 0.025 & 0.000 & 0.003 & 0.000 \\
& $R_{S, p}^{2}$ & 0.007 & 0.015 & 0.001 & 0.014 & 0.010 & 0.000 \\
& $R^{2}$ & 0.008 & 0.018 & 0.026 & 0.014 & 0.013 & 0.001 \\
\hline
\end{tabular}


Table A3: Optimal index composition using Christiano-Fitzgerald filter over the whole sample

The table is Analogous to Table 6 and presents the GMM estimates of the optimal weights to be used for the long-term and short-term components of a single state variable. The difference here is that Christiano-Fitzgerald $(\mathrm{CF})$ filters are run once over the whole sample to estimate the long and short components of state variables. The sample covers April 1953 to June 2011.

\begin{tabular}{|c|c|c|c|c|c|c|c|}
\hline & & $\overline{\mathrm{DSS}}$ & $\overline{\overline{D Y}}$ & $\overline{T S}$ & $\overline{\text { TR }}$ & 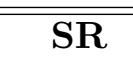 & $\overline{\overline{\mathrm{DG}}}$ \\
\hline \multirow[t]{3}{*}{$\mathrm{CF}(6)$} & $\hat{\phi}$ & $\begin{array}{c}2.151 \\
(0.164)\end{array}$ & $\begin{array}{c}1.531 \\
(0.113)\end{array}$ & $\begin{array}{c}0.370 \\
(0.189)\end{array}$ & $\begin{array}{c}1.901 \\
(0.105)\end{array}$ & $\begin{array}{c}1.591 \\
(0.194)\end{array}$ & $\begin{array}{c}1.741 \\
(1.057)\end{array}$ \\
\hline & $\beta_{1}$ & -0.548 & 0.040 & 0.932 & -0.324 & -0.020 & -0.169 \\
\hline & $\beta_{2}$ & 0.836 & 0.999 & 0.362 & 0.946 & 1.000 & 0.986 \\
\hline \multirow[t]{4}{*}{$\mathrm{CF}(12)$} & $\hat{\phi}$ & 1.911 & 1.541 & 2.741 & 2.051 & 1.821 & 2.451 \\
\hline & & $(0.314)$ & $(0.121)$ & $(0.286)$ & $(0.115)$ & $(0.235)$ & $(0.471)$ \\
\hline & $\beta_{1}$ & -0.334 & 0.030 & -0.921 & -0.462 & -0.248 & -0.771 \\
\hline & $\beta_{2}$ & 0.943 & 1.000 & 0.390 & 0.887 & 0.969 & 0.637 \\
\hline \multirow[t]{4}{*}{$\mathrm{CF}(24)$} & $\hat{\phi}$ & 2.091 & 1.561 & 1.241 & 2.261 & 1.621 & 2.701 \\
\hline & & (1.107) & $(0.166)$ & $(0.326)$ & $(0.129)$ & $(0.264)$ & $(0.402)$ \\
\hline & $\beta_{1}$ & -0.497 & 0.010 & 0.324 & -0.637 & -0.050 & -0.905 \\
\hline & $\beta_{2}$ & 0.868 & 1.000 & 0.946 & 0.771 & 0.999 & 0.426 \\
\hline \multirow[t]{4}{*}{$\mathrm{CF}(36)$} & $\hat{\phi}$ & 1.991 & 1.571 & 2.441 & 2.391 & 1.551 & 2.711 \\
\hline & & $(0.454)$ & $(0.231)$ & $(0.278)$ & $(0.143)$ & $(0.266)$ & $(0.380)$ \\
\hline & $\beta_{1}$ & -0.408 & 0.000 & -0.765 & -0.731 & 0.020 & -0.909 \\
\hline & $\beta_{2}$ & 0.913 & 1.000 & 0.644 & 0.682 & 1.000 & 0.417 \\
\hline \multirow[t]{4}{*}{$\mathrm{CF}(48)$} & $\hat{\phi}$ & 2.051 & 1.701 & 2.361 & 2.561 & 1.591 & 2.681 \\
\hline & & $(0.308)$ & $(0.230)$ & $(0.254)$ & $(0.250)$ & $(0.220)$ & $(0.393)$ \\
\hline & $\beta_{1}$ & -0.462 & -0.130 & -0.711 & -0.836 & -0.020 & -0.896 \\
\hline & $\beta_{2}$ & 0.887 & 0.992 & 0.704 & 0.548 & 1.000 & 0.444 \\
\hline \multirow[t]{4}{*}{$\mathrm{CF}(72)$} & $\hat{\phi}$ & 2.381 & 1.861 & 0.490 & 2.901 & 1.481 & 2.811 \\
\hline & & $(0.884)$ & $(0.227)$ & $(0.271)$ & $(0.230)$ & $(0.306)$ & $(0.335)$ \\
\hline & $\beta_{1}$ & -0.725 & -0.286 & 0.882 & -0.971 & 0.090 & -0.946 \\
\hline & $\beta_{2}$ & 0.689 & 0.958 & 0.471 & 0.238 & 0.996 & 0.324 \\
\hline
\end{tabular}


Table A4: In-sample performance of portfolio strategies using Christiano-Fitzgerald filter over the whole sample

The table is Analogous to Table 7 and presents the in-sample performance of portfolio strategies based on different filter and state variable pairs. The difference here is that Christiano-Fitzgerald (CF) filters are run once over the whole sample to estimate the long and short components of state variables. The sample covers April 1953 to June 2011.

\begin{tabular}{|c|c|c|c|c|c|c|c|}
\hline & & $\overline{\mathrm{DS}}$ & $\overline{\mathrm{DY}}$ & $\overline{\text { TS }}$ & TR & SR & DG \\
\hline \multirow{4}{*}{$\begin{array}{c}\text { Non- } \\
\text { decomposed }\end{array}$} & $\bar{R}$ & 0.003 & 0.003 & 0.004 & 0.004 & 0.003 & 0.003 \\
\hline & $\sigma$ & 0.022 & 0.022 & 0.023 & 0.025 & 0.022 & 0.019 \\
\hline & Sharpe & 0.124 & 0.144 & 0.181 & 0.173 & 0.155 & 0.146 \\
\hline & $\bar{U}$ & 0.0053 & 0.0058 & 0.0066 & 0.0065 & 0.0060 & 0.0057 \\
\hline \multirow{4}{*}{$\mathrm{CF}(6)$} & $\bar{R}$ & 0.005 & 0.011 & 0.004 & 0.013 & 0.006 & 0.003 \\
\hline & $\sigma$ & 0.025 & 0.029 & 0.024 & 0.028 & 0.025 & 0.022 \\
\hline & Sharpe & 0.184 & 0.385 & 0.179 & 0.452 & 0.222 & 0.138 \\
\hline & $\bar{U}$ & 0.0068 & 0.0127 & 0.0066 & 0.0142 & 0.0078 & 0.0057 \\
\hline \multirow{4}{*}{$\mathrm{CF}(12)$} & $\bar{R}$ & 0.005 & 0.011 & 0.004 & 0.011 & 0.005 & 0.003 \\
\hline & $\sigma$ & 0.027 & 0.029 & 0.019 & 0.027 & 0.023 & 0.019 \\
\hline & Sharpe & 0.185 & 0.389 & 0.187 & 0.411 & 0.211 & 0.130 \\
\hline & $\bar{U}$ & 0.0069 & 0.0126 & 0.0065 & 0.0130 & 0.0073 & 0.0054 \\
\hline \multirow{4}{*}{$\mathrm{CF}(24)$} & $\bar{R}$ & 0.005 & 0.008 & 0.004 & 0.009 & 0.006 & 0.003 \\
\hline & $\sigma$ & 0.026 & 0.028 & 0.021 & 0.028 & 0.023 & 0.022 \\
\hline & Sharpe & 0.180 & 0.284 & 0.169 & 0.313 & 0.237 & 0.151 \\
\hline & $\bar{U}$ & 0.0068 & 0.0098 & 0.0062 & 0.0105 & 0.0079 & 0.0059 \\
\hline \multirow{4}{*}{$\mathrm{CF}(36)$} & $\bar{R}$ & 0.005 & 0.007 & 0.004 & 0.007 & 0.006 & 0.004 \\
\hline & $\sigma$ & 0.026 & 0.028 & 0.021 & 0.027 & 0.023 & 0.022 \\
\hline & Sharpe & 0.179 & 0.249 & 0.196 & 0.247 & 0.253 & 0.161 \\
\hline & $\bar{U}$ & 0.0067 & 0.0088 & 0.0068 & 0.0085 & 0.0083 & 0.0061 \\
\hline \multirow{4}{*}{$\mathrm{CF}(48)$} & $\bar{R}$ & 0.005 & 0.006 & 0.005 & 0.005 & 0.006 & 0.004 \\
\hline & $\sigma$ & 0.025 & 0.025 & 0.021 & 0.025 & 0.024 & 0.022 \\
\hline & Sharpe & 0.188 & 0.224 & 0.217 & 0.209 & 0.244 & 0.179 \\
\hline & $\bar{U}$ & 0.0069 & 0.0078 & 0.0073 & 0.0074 & 0.0082 & 0.0065 \\
\hline \multirow{4}{*}{$\mathrm{CF}(72)$} & $\bar{R}$ & 0.004 & 0.004 & 0.004 & 0.005 & 0.005 & 0.004 \\
\hline & $\sigma$ & 0.025 & 0.022 & 0.022 & 0.024 & 0.026 & 0.025 \\
\hline & Sharpe & 0.180 & 0.188 & 0.180 & 0.190 & 0.191 & 0.177 \\
\hline & $\bar{U}$ & 0.0067 & 0.0067 & 0.0065 & 0.0069 & 0.0070 & 0.0066 \\
\hline
\end{tabular}


Table A5: In-Sample Estimation and Performance Using the Hodrick-Prescott Filter over the whole sample

This table is analogous to Table 9 and shows the in-sample estimation and performance of portfolio strategies based on different Hodrick-Prescott filters and state variable pairs. The difference here is that ChristianoFitzgerald (CF) filters are run once over the whole sample to estimate the long and short components of state variables. The sample covers April 1953 to June 2011.

\begin{tabular}{|c|c|c|c|c|c|c|c|}
\hline & & $\overline{\overline{D S}}$ & $\overline{\overline{\mathrm{DY}}}$ & $\overline{\text { TS }}$ & $\overline{\overline{T R R}}$ & $\overline{\overline{\text { SR }}}$ & 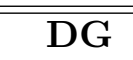 \\
\hline \multirow[t]{8}{*}{$\operatorname{HP}(240)$} & $\hat{\phi}$ & 1.851 & 1.611 & 2.201 & 2.291 & 1.681 & 2.701 \\
\hline & & $(0.297)$ & $(0.163)$ & $(0.169)$ & $(0.110)$ & $(0.276)$ & $(0.463)$ \\
\hline & $\beta_{1}$ & -0.276 & -0.040 & -0.589 & -0.660 & -0.110 & -0.905 \\
\hline & $\beta_{2}$ & 0.961 & 0.999 & 0.808 & 0.752 & 0.994 & 0.426 \\
\hline & $\bar{R}$ & 0.005 & 0.008 & 0.004 & 0.009 & 0.006 & 0.004 \\
\hline & $\sigma_{R}$ & 0.027 & 0.029 & 0.021 & 0.029 & 0.023 & 0.022 \\
\hline & Sharpe & 0.193 & 0.291 & 0.191 & 0.326 & 0.257 & 0.164 \\
\hline & $\bar{U}$ & 0.0071 & 0.0100 & 0.0067 & 0.0109 & 0.0083 & 0.0062 \\
\hline \multirow[t]{8}{*}{$\mathrm{HP}(900)$} & $\hat{\phi}$ & 2.011 & 1.651 & 2.381 & 2.391 & 1.651 & 2.661 \\
\hline & & $(0.355)$ & $(0.189)$ & $(0.263)$ & $(0.134)$ & $(0.256)$ & $(0.489)$ \\
\hline & $\beta_{1}$ & -0.426 & -0.080 & -0.725 & -0.731 & -0.080 & -0.887 \\
\hline & $\beta_{2}$ & 0.905 & 0.997 & 0.689 & 0.682 & 0.997 & 0.462 \\
\hline & $\bar{R}$ & 0.005 & 0.008 & 0.004 & 0.008 & 0.006 & 0.004 \\
\hline & $\sigma_{R}$ & 0.026 & 0.029 & 0.021 & 0.028 & 0.024 & 0.022 \\
\hline & Sharpe & 0.195 & 0.270 & 0.209 & 0.272 & 0.261 & 0.168 \\
\hline & $\bar{U}$ & 0.0072 & 0.0094 & 0.0070 & 0.0094 & 0.0085 & 0.0063 \\
\hline \multirow[t]{8}{*}{$\mathrm{HP}(3600)$} & $\hat{\phi}$ & 2.151 & 1.701 & 2.391 & 2.521 & 1.741 & 2.691 \\
\hline & & $(0.264)$ & $(0.198)$ & $(0.202)$ & $(0.156)$ & $(0.236)$ & $(0.356)$ \\
\hline & $\beta_{1}$ & -0.548 & -0.130 & -0.731 & -0.814 & -0.169 & -0.900 \\
\hline & $\beta_{2}$ & 0.836 & 0.992 & 0.682 & 0.581 & 0.986 & 0.435 \\
\hline & $\bar{R}$ & 0.005 & 0.006 & 0.005 & 0.006 & 0.006 & 0.004 \\
\hline & $\sigma_{R}$ & 0.026 & 0.026 & 0.022 & 0.027 & 0.024 & 0.022 \\
\hline & Sharpe & 0.205 & 0.239 & 0.228 & 0.239 & 0.242 & 0.176 \\
\hline & $\bar{U}$ & 0.0074 & 0.0083 & 0.0075 & 0.0083 & 0.0081 & 0.0064 \\
\hline \multirow[t]{8}{*}{$\operatorname{HP}(14400)$} & $\hat{\phi}$ & 2.331 & 1.731 & 2.481 & 2.631 & 1.721 & 2.761 \\
\hline & & $(0.473)$ & $(0.217)$ & $(0.185)$ & $(0.206)$ & $(0.274)$ & $(0.352)$ \\
\hline & $\beta_{1}$ & -0.689 & -0.159 & -0.790 & -0.873 & -0.150 & -0.929 \\
\hline & $\beta_{2}$ & 0.725 & 0.987 & 0.613 & 0.488 & 0.989 & 0.371 \\
\hline & $\bar{R}$ & 0.005 & 0.005 & 0.005 & 0.005 & 0.005 & 0.004 \\
\hline & $\sigma_{R}$ & 0.025 & 0.024 & 0.022 & 0.025 & 0.025 & 0.023 \\
\hline & Sharpe & 0.189 & 0.208 & 0.226 & 0.206 & 0.213 & 0.175 \\
\hline & $\bar{U}$ & 0.0069 & 0.0073 & 0.0075 & 0.0073 & 0.0075 & 0.0064 \\
\hline
\end{tabular}

